Florida International University FIU Digital Commons

\title{
Capture and Densification of Floating Hydrophobic Liquids by Natural Granular Materials
}

Daria Boglaienko

dbogl003@fiu.edu

DOI: $10.25148 /$ etd.FIDC001735

Follow this and additional works at: https://digitalcommons.fiu.edu/etd

Part of the Environmental Chemistry Commons, Environmental Engineering Commons, Oil, Gas, and Energy Commons, Physical Chemistry Commons, and the Water Resource Management Commons

\section{Recommended Citation}

Boglaienko, Daria, "Capture and Densification of Floating Hydrophobic Liquids by Natural Granular Materials" (2017). FIU Electronic Theses and Dissertations. 3261.

https://digitalcommons.fiu.edu/etd/3261 


\section{FLORIDA INTERNATIONAL UNIVERSITY}

Miami, Florida

\section{CAPTURE AND DENSIFICATION OF FLOATING HYDROPHOBIC LIQUIDS BY NATURAL GRANULAR MATERIALS}

A dissertation submitted in partial fulfillment of the

requirements for the degree of

DOCTOR OF PHILOSOPHY

in

CIVIL ENGINEERING

by

Daria Boglaienko

2017 
To: $\quad$ Interim Dean Ranu Jung

College of Engineering and Computing

This dissertation, written by Daria Boglaienko, and entitled Capture and Densification of Floating Hydrophobic Liquids by Natural Granular Materials, having been approved in respect to style and intellectual content, is referred to you for judgement.

We have read this dissertation and recommend that it be approved.

$\begin{array}{r}\text { Michael Sukop } \\ \hline \text { Piero Gardinali } \\ \hline \text { Shonali Laha } \\ \hline \text { Walter Tang } \\ \hline \text { Yelena Katsenovich } \\ \hline\end{array}$

Date of Defense: February 24, 2017

The dissertation of Daria Boglaienko is approved.

Interim Dean Ranu Jung
College of Engineering and Computing

Florida International University, 2017 
C Copyright 2017 by Daria Boglaienko

All rights reserved. 


\section{DEDICATION}

To my father Viktor Boglaienko, in memoriam. 


\section{ACKNOWLEDGMENTS}

I sincerely thank my adviser, Dr. Berrin Tansel, for her generous support. I appreciate her understanding and patience, inspiration and encouragement. I am grateful for her guidance, insightful discussions, help in the lab, support of my ideas, and valuable revisions and constructive comments for the manuscripts quality improvement.

I am very thankful to Dr. Shonali Laha, Dr. Yelena Katsenovich, Dr. Mike Sukop, Dr. Piero Gardinali, and Dr. Walter Tang for dedicating their time to serve on my dissertation committee and helpful suggestions. I am especially grateful to Dr. Shonali Laha and Dr. Yelena Katsenovich for their sincere care, generous help and encouragement during my study. I appreciate inspiring and creative ideas of Dr. Mike Sukop and his feedback during the preparation and publication of our manuscript. I thank Dr. Piero Gardinali for his time and help with the interpretation of the phenomenon observed during the lab experiments. I am grateful to Dr. Walter Tang for the encouragement of my future way in research and science.

I wish to thank Dr. Yusuf Emirov for his willingness to help and excellent assistance with the facilities at Advanced Materials Engineering Research Institute (AMERI) at FIU.

I acknowledge support of the University Graduate School at FIU through Dissertation Year Fellowship (DYF) award and funding from Gulf of Mexico Research Initiative.

I am grateful to my mother, sister, and grandmother for their immense love, who, despite a distance of thousands of miles, never left me without their kind support and thoughtful care. I thank all my friends I have met on my way of pursuing a doctoral degree. 


\title{
ABSTRACT OF THE DISSERTATION \\ CAPTURE AND DENSIFICATION OF FLOATING HYDROPHOBIC LIQUIDS BY NATURAL GRANULAR MATERIALS
}

\author{
by
}

\section{Daria Boglaienko}

Florida International University, 2017

Miami, Florida

\section{Professor Berrin Tansel, Major Professor}

Densification and submergence of floating crude oil is proposed as a novel oil spills treatment method. Surface application of dry granular materials (e.g., quartz sand, limestone) on top of a floating oil layer increases the density of the floating oil phase/granule mixture and leads to formation of relatively large and stable aggregates with significant amounts of captured oil. The aggregates separate from the floating hydrophobic phase and settle by gravity. Implementation of this method will reduce the impact radius of a spill and its mobility, preventing direct contamination of beaches, coastal flora and fauna.

The major objective of this research was to examine interactions of particles with hydrophobic liquid-water interface from different perspectives. The important characteristics of the process, such as oil removal efficiencies, optimal particle-to-oil ratios and particle size ranges, were experimentally defined. A series of experiments was conducted to investigate aggregation and dissolution rate constants of the submerged hydrophobic liquids in salt water and deionized water, and to study the impact of the surface porosity of the granular particles on oil capture efficiencies. In addition to crude 
oil (South Louisiana crude, MC 252), aggregation volumes of quartz sand with other hydrophobic liquids (alkanes and aromatics) were analyzed in relation to wetting characteristics and physical properties of the liquids. A classification of the main types of oil-particle aggregates was developed based on the formation characteristics of the aggregates. Moreover, under specific conditions, depending on the application rates of the granular materials, unique interactions of the particles with the hydrophobic liquid-water interface were observed and defined (bowl formation and roping).

These concepts can be utilized to control surface mobility of floating oils, especially during the initial stages of an oil spill, while the oil layer is intact, and when other treatment methods may not be suitable near coastal areas, where transport of floating oils can significantly impact coastal ecosystems. 


\section{TABLE OF CONTENTS}

CHAPTER

PAGE

I. INTRODUCTION 1

II. INSTANTANEOUS STABILIZATION OF FLOATING OILS BY SURFACE APPLICATION OF NATURAL GRANULAR MATERIALS (BEACH SAND

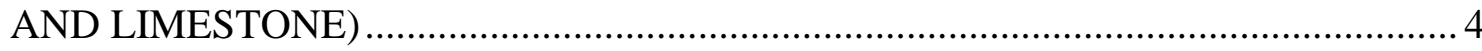

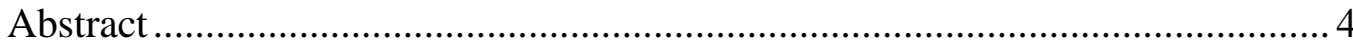

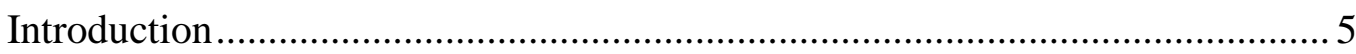

Theoretical Analyses.............................................................................. 8

Materials and Methods............................................................................ 10

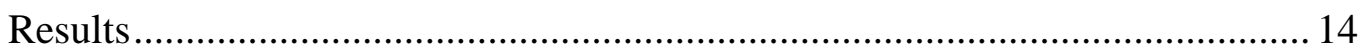

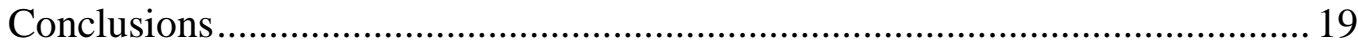

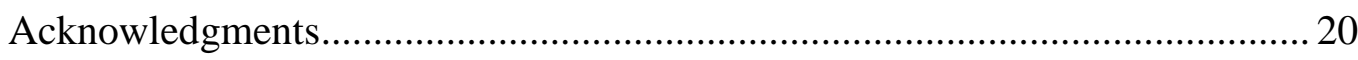

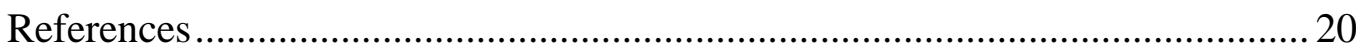

III. PARTITIONING OF FRESH CRUDE OIL BETWEEN FLOATING, DISPERSED AND SEDIMENT PHASES: EFFECT OF EXPOSURE ORDER TO DISPERSANT AND GRANULAR MATERIALS ................................................... 22

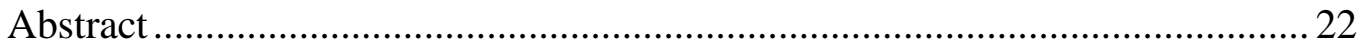

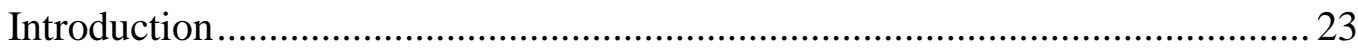

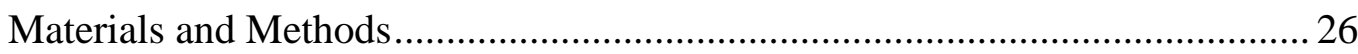

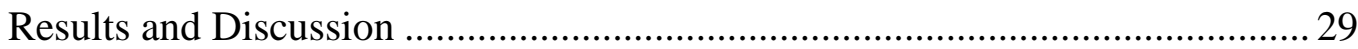

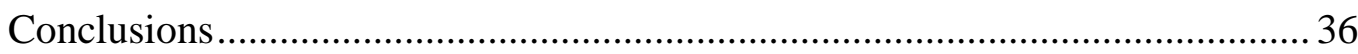

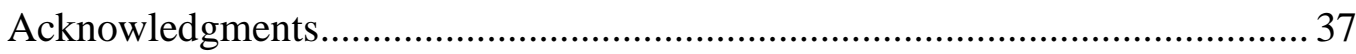

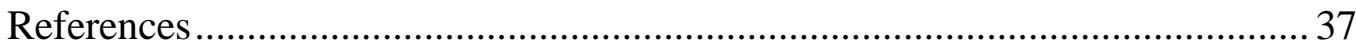

IV. GRANULAR ENCAPSULATION OF LIGHT HYDROPHOBIC LIQUIDS

(LHL) IN LHL-SALT WATER SYSTEMS: PARTICLE INDUCED

DENSIFICATION WITH QUARTZ SAND......................................................... 40

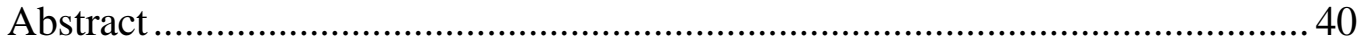

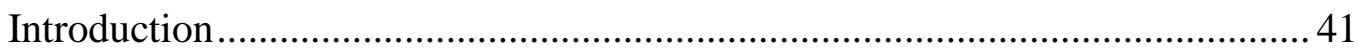

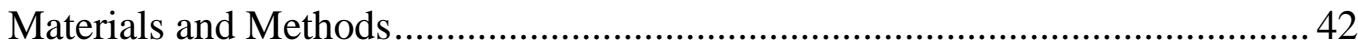

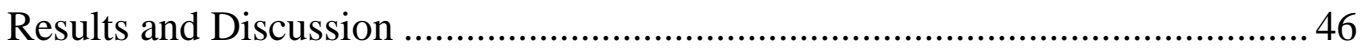

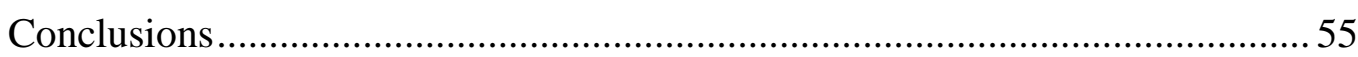

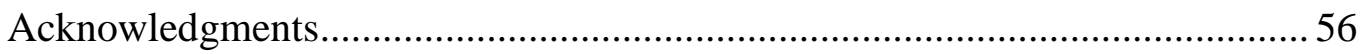

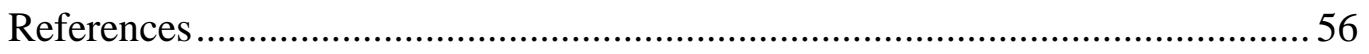


V. ENCAPSULATION OF LIGHT HYDROPHOBIC LIQUIDS WITH FINE QUARTZ SAND: PROPERTY BASED CHARACTERIZATION AND

STABILITY IN AQUEOUS MEDIA WITH DIFFERENT SALINITIES ...................59

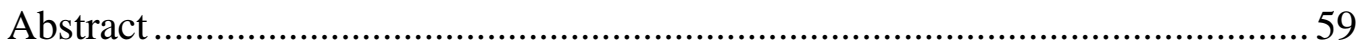

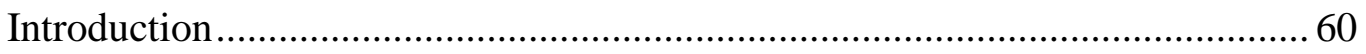

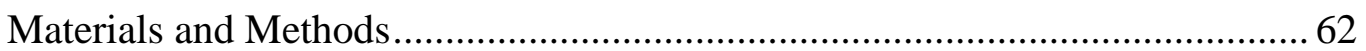

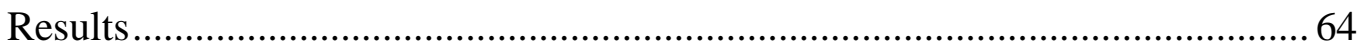

Discussion and Conclusion ........................................................................ 71

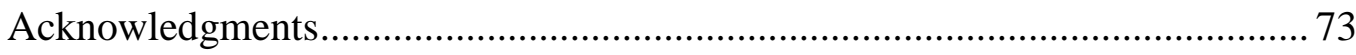

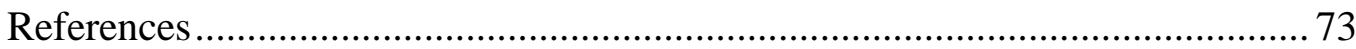

VI. GRAVITY INDUCED DENSIFICATION OF FLOATING CRUDE OIL BY GRANULAR MATERIALS: EFFECT OF PARTICLE SIZE AND SURFACE

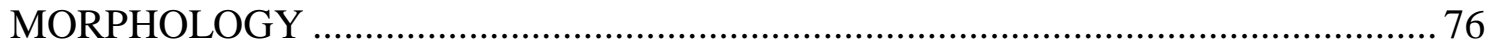

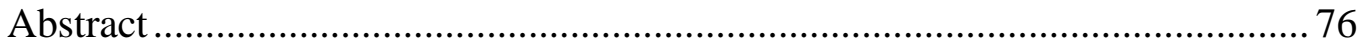

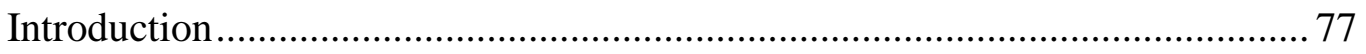

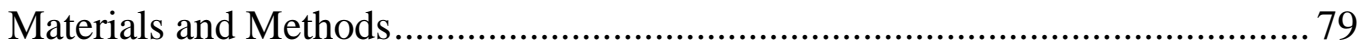

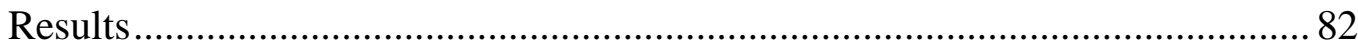

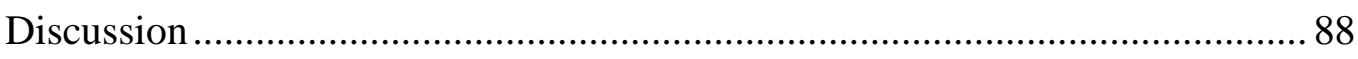

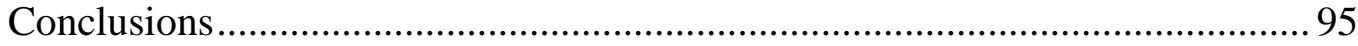

Acknowledgments................................................................................. 96

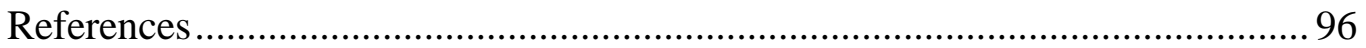

VII. SUBMERGENCE PATTERNS OF FLOATING CRUDE OIL BY

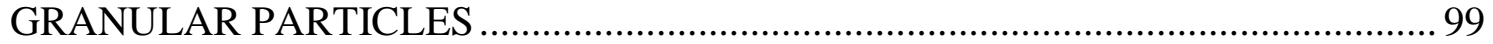

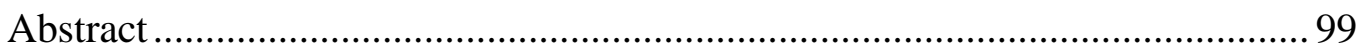

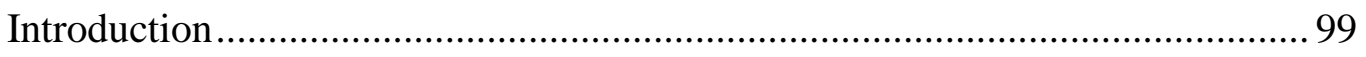

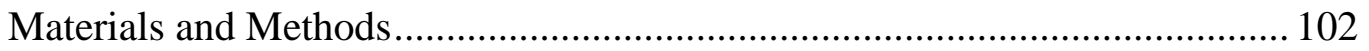

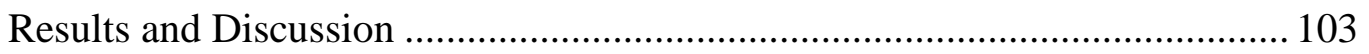

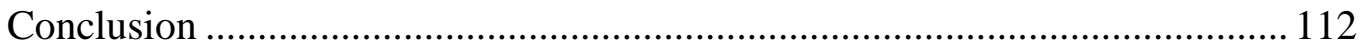

Acknowledgments................................................................................. 113

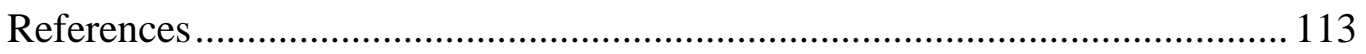

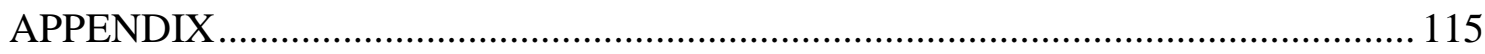

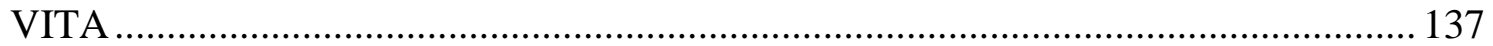




\section{LIST OF TABLES}

TABLE

PAGE

\section{CHAPTER II}

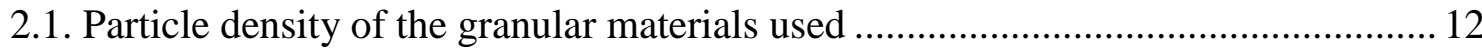

2.2. Characteristics of the materials used............................................................ 12

2.3. Oil capture efficiency $(E)$ of granular materials used ..................................... 17

2.4. Experimental $k$ values (ratio of mass of oil-particle aggregates formed to mass

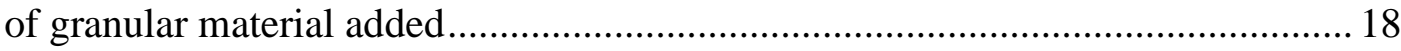

\section{CHAPTER III}

3.1. Partitioning of floating oil between float and sediment phases relative to concentration in water phase

\section{CHAPTER IV}

4.1. Contact angle estimates, density, liquid-water interfacial tension, and aggregated volume of the selected HCs (average of two measurements)

\section{CHAPTER V}

5.1. Aggregation coefficient $K_{a g}(\leq 0.5 \mathrm{~mL})$ and percent of the aggregated volume from the initial $(0.5 \mathrm{~mL})$ for each HL. Data are averages of two sets; standard deviation is given in parenthesis. Layer thickness to particle diameter ratio is $>4: 1$

5.2. Selected properties of the hydrophobic liquids 65

5.3. Dissolution rate constants $\left(\right.$ day $\left.^{-1}\right)$ of the volume loss in aggregated globules of HL (time period $=10$ days)

\section{CHAPTER VI}

6.1. Granular materials: particle size and bulk density; composition by Energy Dispersive Spectroscopy (EDS) and images by Scanning Electron Microscopy 
(SEM): X15 for medium quartz sand, fine quartz sand, and beach sand; X20 for limestone and clay

6.2. Porosity analyses of the granular materials (SEM - ImageJ) and aggregation

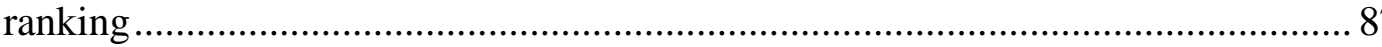

6.3. Comparison of experimental conditions in studies on submerging floating oils with granular particles with raft and slurry formation......................................90

\section{CHAPTER VII}

7.1. Submergence patterns of floating crude oil with granular particles.

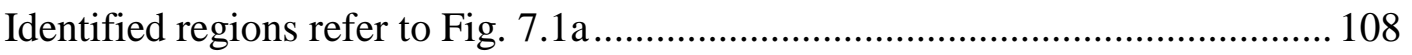




\section{LIST OF FIGURES}

FIGURE

PAGE

\section{CHAPTER II}

2.1. Formation of oil-particle aggregates ............................................................

2.2. Transitioning of the floating oil phase from stable raft to unstable raft phase by addition of granular particles.......................................................................... 8

2.3. Granular particles used in the experiments: a. Medium limestone; b. Fine limestone; and c. Fine quartz

2.4. Formation of oil-sand aggregates: a. Floating oil; $b$. Formation and separation of particle-oil aggregates; and c. Particle-oil aggregates separated by coarse filtration (crude oil-fine quartz aggregates)............................................................. 13

2.5. Comparison of oil-particle aggregate formation with different granular particles with increasing particle/oil ratios. Top row: medium limestone; middle row: fine limestone; and bottom row: fine quartz

2.6. Capture of floating oil by granular materials: a. Change in the amount of oil captured in the aggregate phase in relation to amount of granular material added; and $b$. Oil removal efficiency in relation to the ratio of granular material added to floating oil present.

2.7. Variation oil capture coefficient $(k)$ of the granular aggregates in relation to the ratio of granular material added to floating oil present

\section{CHAPTER III}

3.1. Experimental design (O: crude oil, D: dispersant, S: Solid granular material, GM: granular material).

3.2. Phase distribution of oil depending on the presence and absence of the dispersant Corexit 9500A and granular materials (error bars: \pm 1 SD): a. Fine quartz used as solid granular material; and $\mathrm{b}$. Medium limestone used as solid granular material

3.3. Profiles of mixtures illustrating the dispersed oil for different series 34 
3.4. Bottom view of the mixtures illustrating oil capture by granular materials (as sediments) for different series

3.5. Dispersion effectiveness for different series that utilized granular materials; Tukey HSD post hoc $(p<0.05)$; error bars: \pm 1 SD. Significance: ODS-SOD $p=0.053$; ODS-OSD $p=0.001$; ODS-OS $p=0.000$; SOD-OSD $p=0.220$; SOD-OS $p=0.000$; OSD-OS $p=0.032$ (Tukey HSD post hoc test)

\section{CHAPTER IV}

4.1. Solid-liquid-liquid contact angle measurements: (1) quartz crystal; (2) drop of decane; and (3) salt water medium

4.2. LHL-quartz sand interactions in aqueous solutions: a. and b. Aggregation of tetradecane (without dye and with dye) with fine quartz sand; and c. Quartz sand-encapsulated tetradecane globules

4.3a. Comparison of wetting characteristics of decane and toluene on quartz surface; exposed to air (total wetting and spreading) and immersed in salt water (hydrophobic behavior)

4.3b. Wetting characteristics of salt water on quartz surface (air as the medium)

4.3. Wetting characteristics on quartz surface and contact angle measurements

4.4a. Formation of encapsulated light hydrophobic globules after addition of quartz sand in LHL-salt water systems

4.4b. Comparison of the encapsulated globules formed in LHL-salt water systems after addition of quartz sand. LHLs with higher densities formed larger globules with less sand coverage: a. 2-cholorotoluene $\left(\mathrm{d}=1077 \mathrm{~kg} / \mathrm{m}^{3}\right)$; b. Ethylbenzene $\left(\mathrm{d}=865 \mathrm{~kg} / \mathrm{m}^{3}\right)$; and c. Decane $\left(\mathrm{d}=728 \mathrm{~kg} / \mathrm{m}^{3}\right)$

4.4. Encapsulation of hydrophobic liquids with fine quartz sand

4.5. Correlation of the aggregated and gravity-separated volume of the hydrophobic liquids with the quartz-hydrophobic liquid contact angle (measured in salt water); $r^{2}=0.56$

4.6. Variation of globule density with its radius $(\mathrm{mm})$ and surface coverage (\%) by fine quartz particles. Blue, red, and green lines: 70,50 , and $30 \%$ of sand coverage of a globule respectively. Globules with densities greater than the 
water phase (shown as a horizontal black line) settle. Vertical lines show the range of observed globule size formed during the experiments. Modelling was made for the globules of fresh formation (gas phase on top of a globule was not accounted for)

\section{CHAPTER V}

5.1. Materials and aggregated globules: a. Fine quartz sand; b. Erlenmeyer flask with salt water $(100 \mathrm{~mL})$ and floating layer of a dyed HL $(0.5 \mathrm{~mL})$;

c. Conceptual schematic of adding sand to HL slick; d. and e. Floating and submerged globules of hexadecane aggregated with fine quartz sand; $f$. and g. Floating and submerged globules of $m$-xylene aggregated with fine quartz sand; and h. Close up view (top) of the submerged globules of m-xylene aggregated with fine quartz sand

5.2. Volume of the aggregated HLs in salt water (SW) and deionized water (DIW) versus selected physical properties. Selection in ellipse indicates alkanes (decane, tetradecane, and hexadecane); in rectangle - BTEX group (benzene, toluene, ethylbenzene, and m-xylene); 2-chlorotoluene is the one outside groupings

5.3. Observed and calculated mass of aggregates of the HLs in SW ( $0.5 \mathrm{~mL}$ of HL and $1 \mathrm{~g}$ of fine quartz sand; layer thickness to particle diameter ratio is $>4: 1$ ); error bars show \pm 1 SD. a. Pentane; b. Silicone oil (viscosity $50 \mathrm{cSt}$ ); and c. Crude oil (South Louisiana crude) are added as additional HLs for validation of the equations 2-4. Among the eight initially tested HLs, d. 2-chlorotoluene showed the biggest range in size distribution (globule size) and it was the most stable in terms of its shape and captured volume in the globules (air bubbles are clearly visible on top of the globules)

5.4a. Decrease in total volume of the aggregated globules of HLs during 10 days. Data are averages of two series of experiments conducted with salt water (SW) and two series in deionized water (DIW)

5.4b. Estimated dissolution rates in relation to solubility of the HLs 70

5.4. Time dependency of the aggregated volume of globules and dissolution rates $\left(\right.$ day $\left.^{-1}\right)$ of the HLs ..... 


\section{CHAPTER VI}

6.1a. Profile views of the aggregation of crude oil and different materials: Oil as the control; Clay1: right upon clay application; Clay2: 1.5 min after the application; FQ1: fine quartz sand, slow addition; FQ2: fine quartz sand, fast addition; BS: beach sand; MQ: medium quartz sand; Lst: limestone; and CLst: coarse limestone.

6.1b. Close up views on the aggregated materials with crude oil (bottom of a flask). Clay; BS: beach sand; FQ1: fine quartz sand, slow addition; FQ2: fine quartz sand, fast addition; MQ: medium quartz sand; and Lst: limestone.

6.1. Aggregation of SLC oil and different granular materials 85

6.2. SEM images for the granular materials observed at the magnification X6000 (scale bar corresponds to $1 \mu \mathrm{m}$ ): a. Medium quartz sand; b. Fine quartz sand; c. Beach sand; d. Limestone; and e. Clay

6.3. Variation of oil capture efficiency in relation to specific pore surface area (a) and pore size (b)

6.4. Comparison of aggregation of decane (dyed with Sudan IV) and SLC with clay:

a. Decane immediately after clay addition; b. SLC oil after clay addition; and

c. Decane 3 minutes after clay addition

6.5. Different types of aggregates formed with granular particles and hydrophobic liquids. Complete encapsulation formed with a mixture of alkanes (decane, tetradecane, and hexadecane) and beach sand particles, raft formed with 2-chlorotoluene and beach sand particles, transitional slurry formed with SLC and clay, and slurry formed with limestone

\section{CHAPTER VII}

7.1. Submergence patterns and granular interactions: a. Conceptual submergence pattern diagram for South Louisiana crude oil and quartz sand (average particle sizes 0.28 and $0.72 \mathrm{~mm}$ ), regions are: I pass through, II bowl formation, III aggregate following bowl formation, IV small aggregates, V large aggregates, and VI roping state. Division lines between different submergence states are approximate. b. Behavior of granular particles in different states and interaction with oil: (a) immobile oil shell during pass through state (very thin oil film on the particles), (b) elongation of mobile oil shell due to viscous interactions during submergence in roping state, (c) mobile oil shell interactions during 
large aggregate formation, and (d) immobilization of mobile shell during bowl formation

7.2. Submergence patterns of granular particles: a. Quartz particles with $0.28 \mathrm{~mm}$ average size at $0.20 \mathrm{~g} / \mathrm{s}$ application rate; b. Quartz particles with $0.72 \mathrm{~mm}$ average size at $0.32 \mathrm{~g} / \mathrm{s}$ application rate; c. Quartz particles with $0.28 \mathrm{~mm}$ average size at $1.58 \mathrm{~g} / \mathrm{s}$ application rate; and d. Quartz particles with $0.72 \mathrm{~mm}$ average size at $0.87 \mathrm{~g} / \mathrm{s}$ application rate. The same color represents the submergence states during each test condition (for example, aggregate formation-bowl-pass through; or bowl-pass through; or pass through only, depending on the oil thickness at the starting point). Scale bar on photos: $4 \mathrm{~cm}$. Colors identifying each region are the same as defined in Fig. 7.1a

7.3. Occurrence of bowl state: a-c. Top and side views of bowl formation; d, e. Bowl formation with decane (dyed with Sudan red) and quartz particles $(0.28 \mathrm{~mm})$; (e) submerged bowl (paste-like aggregate); near spherical globules below surface are the raft type of hydrophobic liquid-particle aggregates. f. Strength and elasticity of the floating aggregate that formed at the critical oil thickness which resulted in bowl formation (floating) and large aggregates that submerged when the oil layer was above the critical thickness (submerged) prior to bowl

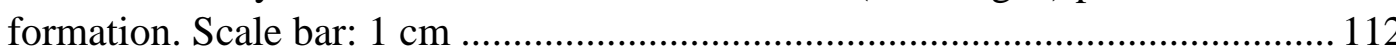




\section{CHAPTER I}

\section{INTRODUCTION}

Whenever there is an environmental issue - pollution, resource exploitation and overuse, need for environmental protection and control - the methods we employ and strategies we choose are based on a fundamental principle of proportionality to our own needs and resources, thus, creating a demand for affordable solutions and accessible technologies. In addition, the more environmental-friendly a solution is, the less trouble it leaves behind, eliminating many possible negative consequences and expenses. This dissertation discusses one such solution - environmentally friendly, inexpensive, affordable, and accessible in any part of the world.

An oil spill is a kind of environmental issue that arises wherever drilling and production, transportation and refining, delivery and distribution of oil occur. It is a quotidian and ordinary event in the overall petroleum industry's functioning and development. It can be local within a factory zone or a pipeline area, or it can reach the international scale, polluting the coastlines of several countries (e.g., Ixtoc I in the Gulf of Mexico, 1979-1980). The ubiquitous nature of the petroleum industry, and petroleum's pervasive presence in numerous consumer products, makes oil spills a serious global environmental issue.

Moreover, there are natural oil flows, so-called oil seeps, bringing oil up to the seafloor or to the land surface; these stimulate significant natural biodegradation processes at the particular area. Thus, localization and immobilization of an oil spill in order to prevent its further spread and to avoid coastline contamination can be considered as a 
preferential strategy in oil spill response and mitigation. Aggregation of oil with natural granular particles, such as quartz sand or limestone, is the process that captures, densifies and transports oil from a floating phase to a submerged one, and it is the approach that avoids the introduction of additional xenobiotic compounds to the marine environment. By reducing the radius of a spill we avert the oil contamination of shoreline flora and fauna; and by transporting oil to the bottom of a water basin, we rely on the natural biodegradation processes, similar to the environmental fate of natural oil seeps.

The presented process can be used for an oil spill capture and mobility reduction with the following recommendations: granular particles of the size range $0.1-2.0 \mathrm{~mm}$ in average diameter; effective ratio is $2 \mathrm{~g}$ of particles to $1 \mathrm{~g}$ of oil; mixtures of particles should be dry and applied at rates around $1.5 \mathrm{~g} / \mathrm{s}$ and higher with no difference in capture efficiency for fresh or salt water basins.

The main benefits of this method are its simplicity (application of a dry granular material on top of a floating oil), availability (beach sand, pure quartz sand, and limestone), and accessibility (common natural materials all over the earth, no manufacturing or marketing needed). In addition, granules can be used for densification of any floating hydrophobic phase (alkanes, aromatic hydrocarbons, silicone oils, machine oils, etc.).

This dissertation consists of seven published articles, each representing a chapter (one of the articles is in the Appendix). The chapters answer the research questions that can be generalized as follows:

- floating oil capture efficiencies by different materials (Chapter II);

- removal of floating oil as a captured and settled phase in comparison to oil dispersion by Corexit dispersant (Chapter III); 
- modeling of settling behavior of aggregated globules (Chapter IV);

- aggregation volumes of hydrophobic liquids (alkanes and aromatics) with quartz sand in relation to wetting characteristics (Chapter IV) and physical properties of liquids (Chapter V);

- aggregation coefficients and dissolution rate constants of the submerged hydrophobic liquids in salt water and deionized water (Chapter V);

- impact of surface porosity of the granular particles on oil capture efficiencies and the optimal particle size range that allows effective aggregation (Chapter VI);

- classification of the main types of oil-particle aggregates (Chapter VI); and

- occurrence of different submergence and aggregation patterns under varying application rates of the granular materials (Chapter VII).

Additionally, factors that influence preferential positioning of the granular materials at hydrophobic liquid-water interfaces were analyzed and are presented in the Appendix. 


\title{
CHAPTER II
}

\section{INSTANTANEOUS STABILIZATION OF FLOATING OILS BY SURFACE APPLICATION OF NATURAL GRANULAR MATERIALS (BEACH SAND AND LIMESTONE)}

\author{
Boglaienko, D., and Tansel, B. 2015. Marine Pollution Bulletin, 91 (1): 107-112.
}

DOI: 1016/j.marpolbul.2014.12.020

\begin{abstract}
When granular materials are applied to hydrophobic liquids floating over another liquid (i.e., water), particles form aggregates which can be separated from the floating phase. This concept can be used for controlling mobility of floating oils, especially after oil spills near coastal areas. The objectives of this research were to characterize oil capture efficiency and determine effectiveness of particles for converting the floating phase to a heavier phase for effective separation. Experiments were conducted with South Louisiana crude oil contaminated salt water, limestone and quartz sand. Although the oil removal efficiency increased with increasing amount of granular material applied, it did not increase linearly. About $50 \%$ of the floating oil was removed by aggregates regardless of the material used when granular material to floating oil ratio was about $1 \mathrm{~g} / \mathrm{g}$. The aggregates separated had higher amounts of oil content when smaller amounts of granular materials were added.
\end{abstract}




\section{Introduction}

Addition of a small amount of liquid to dry granular particles changes their behavior significantly as the liquid forms bridges between the particles. The process may be described by induced cohesion between particles due to the surface tension of the liquid (Fingerle and Herminghaus, 2008; Mitarai and Nori, 2006; Herminghaus, 2005; Iveson and Vallance, 2001); as adhesion takes place due to solid and liquid bridges (Simons, 1996; Rossetti et al., 2003); or by capillary forces (Abkarian et al., 2013); or due to free surface energy changes (Bhushan, 2003). Similarly, the opposite process (i.e., adding granular particles to liquid) also results in changes in the characteristics of the liquid when small amounts of granular material are added into the liquid phase. The primary bonding mechanisms between the granular particles and liquid include: 1. adhesion and cohesion forces in the liquid phase molecules with granular particles; 2. interfacial forces in liquid phase with granular particles; 3. formation of liquid bridges and coating of granular particles; 4. attractive forces between granular particles and liquid molecules; and 5. physical interlocking of hydrophobic liquid by the granular particles due to surface tension.

Researchers Abkarian et al. (2013) define such oil-particle aggregates as rafts that are a close-packed layer of particles in oil formed due to long-range capillary attractions. The raft sinks when the number of particles is large enough to compensate the lower density of oil and the balance between buoyancy force and capillary force is changed.

Crude oil sedimentation by adhesion of hydrophobic liquid after collusion with solid particles is a natural mechanism that can be utilized for phase separation of floating oils. The phenomenon is similar to the interaction of suspended particles and their flux 
through the hydrophobic phase which occur in the environment. In muddy waters with sediment concentration over $0.5 \mathrm{~kg} / \mathrm{m}^{3}$, oil sedimentation may exceed normal dispersion (Lehr et al., 2010). According to Payne et al. (1987), at suspended particle concentration 1-10 mg/L, no oil sedimentation occurs, at 10-100 mg/L, oil sedimentation happens in the sufficient turbulence mixing, and at $>100 \mathrm{mg} / \mathrm{L}$ massive oil transport may occur. Payne et al. (1987) developed a mathematical model for oil droplets and suspended particulate matter interaction kinetics using an experimental setup consisting of a stirred tank with suspended oil droplets and particulate matter. Two main mechanisms considered were the interaction of oil sorption on suspended solids and oil collision with suspended solids. Similarly, collusion of granular particles with a floating oil layer (rather than the dispersed oil droplets) can be an effective mechanism for transformation of floating oils into an immobile phase by utilizing the sticky nature of oil on the solid particles when the granular particles mix with the floating oil layer.

For a highly hydrophobic liquid (e.g., oil) floating over another liquid (e.g., water), addition of granular particles into the hydrophobic floating phase gradually increases the density of the floating phase. As the hydrophobic liquid retains the granular particles, particles form aggregates due to the cohesive forces as illustrated in Fig. 2.1. As the density of the oil-particle aggregates reach a critical point, the oil-water aggregates separate from the floating phase while retaining a significant amount of the hydrophobic phase as presented in Fig. 2.2. This concept can be used for controlling surface mobility of floating oils, especially during the initial stages while the floating oil layer is intact and dispersant application may not be suitable especially near coastal areas where transport of floating oils can significantly impact coastal ecosystems. Once the oil-particle phase separates, 
additional measures can be taken for remediation of the sediments or separation of the aggregates as they form.

The objectives of this research were to determine:

1. Characteristics of granular materials suitable for capturing floating oils at sea;

2. Identify natural materials which can be suitable for controlling transport of floating oils;

3. Determine suitable oil/granular material ratios in relation to particle size;

4. Characterize oil capture efficiency in relation to granular particle characteristics; and

5. Determine effectiveness of natural granular particles for converting the floating phase to a heavier phase for effective separation.

Experiments were conducted with South Louisiana Crude oil contaminated salt water. Limestone sand with medium and fine particle sizes and quartz sand with fine particle size were used. The characteristics of the granular particles were controlled by sieving through No. 4, 16, 50, and 270 sieves. The oil-particle aggregates were separated by filtering the solutions through a coarse fiberglass filter to determine the capture efficiencies of the granular particles in relation to the oil-particle ratios (w/w).

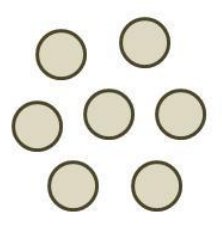

Granular particles

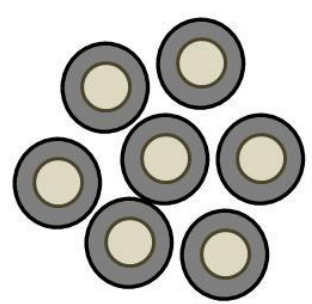

Particles coated with oil

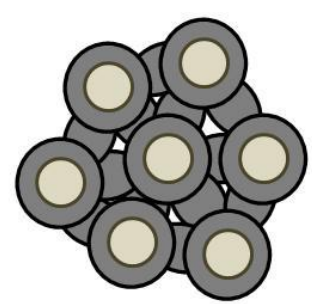

Bridging and aggregation (oil-particle-water)

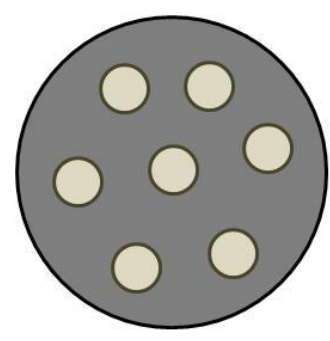

Particle-oil aggregate (oil-particle)

Figure 2.1. Formation of oil-particle aggregates. 


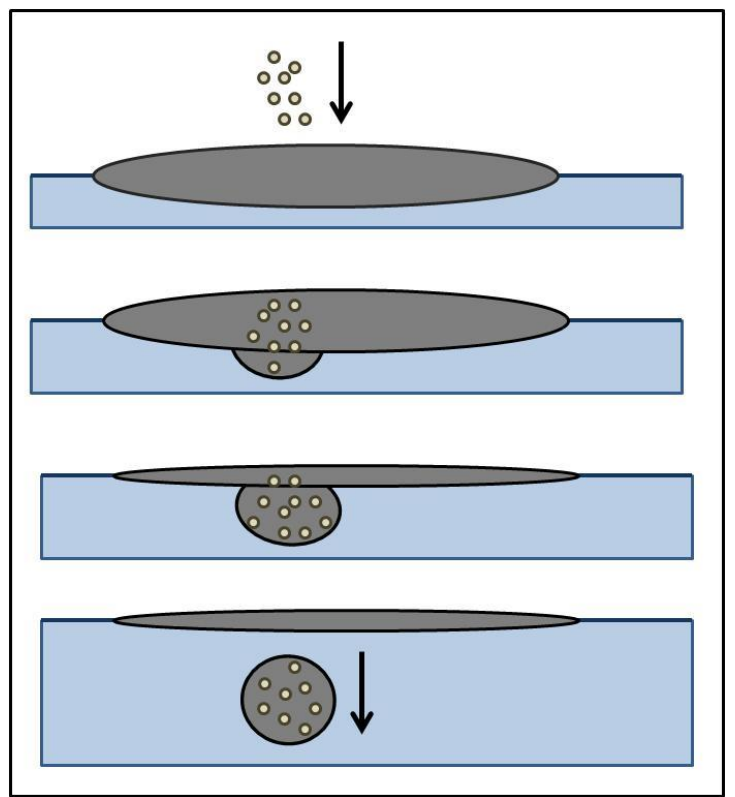

Figure 2.2. Transitioning of the floating oil phase from stable raft to unstable raft phase by addition of granular particles.

\section{Theoretical Analyses}

The rate of collision and adhesion of an oil droplet onto a suspended particle to produce an oil-particulate agglomerate (i.e., loss or settling of free oil droplet) can be estimated by the following equation (Payne et al., 1987):

$$
R=k C_{o} C_{p}
$$

where $R$ is the collision rate, $C_{o}$ is oil-droplet concentration, $C_{p}$ is total particulate concentration, and $k$ is the rate constant for the reaction which depends on the turbulence or energy dissipation rate.

A similar equation for collision frequency per unit volume is provided by Kusters et al. (1996):

$$
J_{i j}=\beta_{i j} n_{i} n_{j}
$$


where $\beta_{i j}$ is the collision frequency function, and $n_{i}$ and $n_{j}$ are the number particles per unit volume.

The rate constant $k$ and collision frequency $\beta_{i j}$ can be described by the flow turbulence (Payne et al., 1987; Kusters et al., 1996; Kaku et al., 2006).

Equation (1) can be written as a material balance expressed in relation to change during incremental period of time (Payne et al., 1987):

$$
\frac{d C o}{d t}=-k G C_{o} C_{p} \quad \text { or } \quad \frac{d n_{i}}{d t}=-k G n_{i} n_{j}
$$

where $k$ is the overall rate constant (i.e., 'sticking' efficiency dependency on the particle size). $G$ denotes the shear rate; $G=(\varepsilon / v)^{0.5}$, where $\varepsilon$ is the turbulent energy dissipation per unit mass per unit time and $v$ is the kinematic viscosity.

During the experiments, it was observed that particle-oil collision occurred instantly after application of the granular particles to the floating oil layer. All solid particles were distributed on the top of the floating oil layer. Thus, collision frequency can be considered to be equal to the number of particles that hit the oil slick:

$$
J_{i j}=n_{j}
$$

Then, the material balance can be rewritten as:

$$
M_{a}=k M_{p}
$$

where $M_{a}$ is the mass of oil-particle aggregate phase $(\mathrm{g}), M_{p}$ is the mass of solid particles $(\mathrm{g})$, and $k$ is the dimensionless oil capture coefficient of the particles into the aggregate phase. Higher values of $k$ indicate more effective capture of oil by the particles from the floating phase into the aggregate phase. 
The aggregation efficiency, $E$, can be estimated from the amount of granular material applied, the initial mass of floating oil $\left(M_{o}\right)$, and the mass of oil that settled in oilparticle aggregates $\left(M_{s o}\right)$ by the following equations:

$$
\begin{aligned}
& E=\frac{M_{s o}}{M_{o}} 100 \% \\
& M_{s o}=M_{a}-M_{p}
\end{aligned}
$$

Combining equations (5), (6) and (7), amount of granular particles to be applied to aggregate floating oil at a desired removal efficiency, $E(\%)$, can be estimated by the following equation:

$$
M_{p}=\frac{E M_{o}}{100(k-1)}
$$

\section{Materials and Methods}

South Louisiana crude (SLC) oil was obtained from the BP America Production Company (Houston, TX). Synthetic sea salt "Instant Ocean" (Aquarium Systems, Mentor, $\mathrm{OH})$ was used at a concentration of $34 \mathrm{~g} / \mathrm{L}$ to prepare the salt water solutions. Experiments were conducted with limestone with medium and fine particle size ranges, and quartz sand with fine particle size range. Tables 2.1 and 2.2 provide the characteristics of the materials used. Granular samples were sieved through sieves No. 4, 16, 50, and 270 to control particle size as shown in Table 2.2 and Fig. 2.3.

A volume of $1 \mathrm{~mL}$ crude oil was added to $100 \mathrm{~mL}$ saltwater in $125 \mathrm{~mL}$ Erlenmeyer flask using 5-mL volumetric pipette. Limestone and quartz sand were weighted $( \pm 0.001$ g) in predetermined amounts of 0.5, 1.0, 1.5, 2.0, and $3.5 \mathrm{~g}$. An Erlenmeyer flask was 
placed in the orbital shaker $(150 \mathrm{rpm})$ to simulate gentle mixing and low sea state turbulence (Kaku et al., 2006). A predetermined amount of limestone or quartz was added while the flask was shaking. Oil-particle aggregation and precipitation occurred almost instantly after the addition of the granular particles onto the oil layer, within 10-25 sec; Fig. 2.4a,b. The flask was removed from the orbital shaker and the solution was filtered through the coarse nonwoven fiberglass filter to separate oil-particle aggregates (Fig. 2.4c).

The solution passed through the fiberglass filter very fast and easily. The oilparticle aggregates were captured by the filter. The capture aggregates were stable and retained their integrity during handling. The filter was weighted before and after the filtration step. The weight of the wet filter (to account for the water presence during the filtering) was subtracted from the weight of filter with oil aggregates. The amount of oil captured by the particles was subtracted from the weight of filtered aggregate and the residual amount of oil (g) was considered as removed by the particles (i.e., settled oil). The oil removal efficiency, $E$, was determined according to formula (6), where $M_{o}=0.9 \mathrm{~g}$ (as $1 \mathrm{~mL}$ of oil). Experiments were repeated between 3-4 times depending on the variations observed. Table 2.3 presents the oil capture efficiency by the particles. Oil-particle aggregates remained stable and retained their integrity even after several days after the experiments. The fact of the aggregates stability despite stirring and surface waves is also supported by the observations of Abkarian et al. (2013). 
Table 2.1. Particle density of the granular materials used.

\begin{tabular}{lc}
\hline Granular material & No. of particles per g \\
\hline Medium limestone & $2.9 \times 10^{3}$ \\
Fine limestone & $1700 \times 10^{3}$ \\
Fine quartz & $600 \times 10^{3}$ \\
\hline
\end{tabular}

Table 2.2. Characteristics of the materials used.

\begin{tabular}{|c|c|}
\hline Material and Property & Typical value \\
\hline \multicolumn{2}{|l|}{ South Louisiana crude oil } \\
\hline API gravity & $32.72^{\mathrm{a}}$ \\
\hline Density (g/mL) & $0.856\left(15^{\circ} \mathrm{C}\right)^{\mathrm{a}}$ \\
\hline Dynamic viscosity (cP) & $10.1\left(15^{\circ} \mathrm{C}\right)^{\mathrm{a}}$ \\
\hline \multicolumn{2}{|l|}{ Limestone } \\
\hline Medium particle size & $<2.00 \mathrm{~mm}$ and $>0.300 \mathrm{~mm}$ \\
\hline Fine particle size & $<0.300 \mathrm{~mm}$ and $>0.075 \mathrm{~mm}$ \\
\hline Bulk density $\left(\mathrm{g} / \mathrm{cm}^{3}\right)$ & $2.5(\text { ave })^{\mathrm{b}}$ \\
\hline \multicolumn{2}{|l|}{ Quartz } \\
\hline Particle size & $<0.300 \mathrm{~mm}$ and $>0.075 \mathrm{~mm}$ \\
\hline Bulk density $\left(\mathrm{g} / \mathrm{cm}^{3}\right)$ & $2.2(\text { ave })^{\mathrm{b}}$ \\
\hline
\end{tabular}




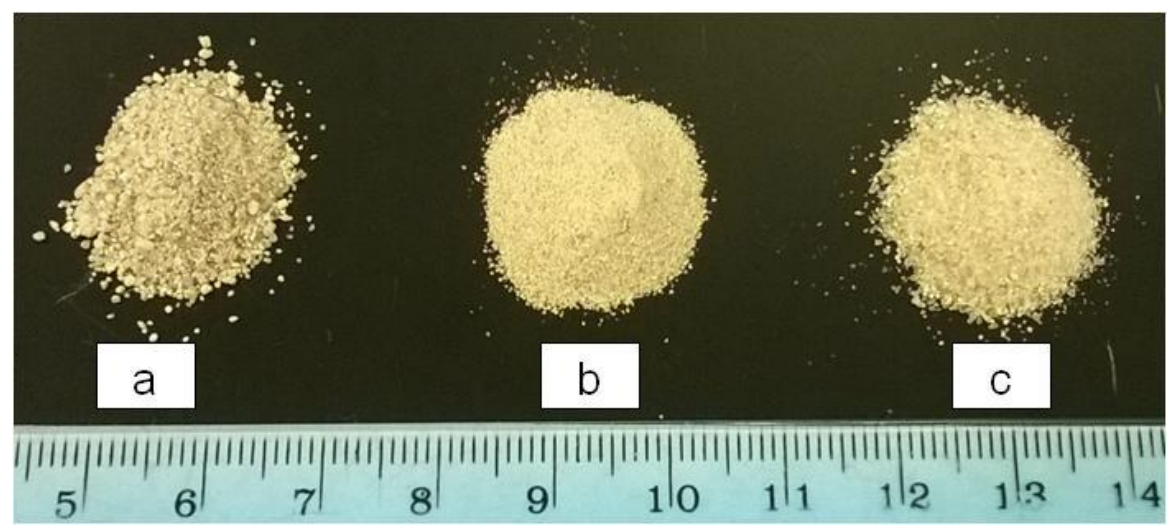

Figure 2.3. Granular particles used in the experiments: a. Medium limestone; b. Fine limestone; and c. Fine quartz.

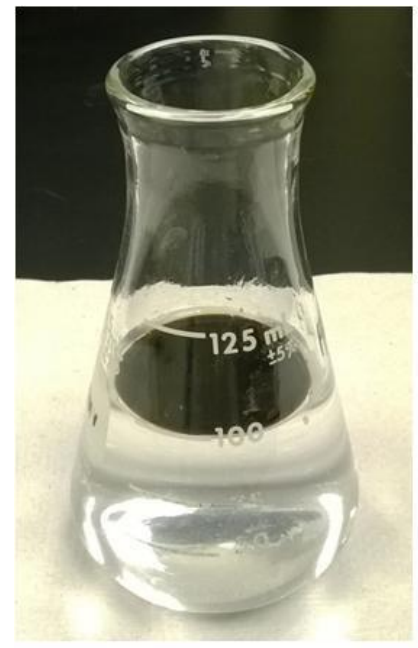

a

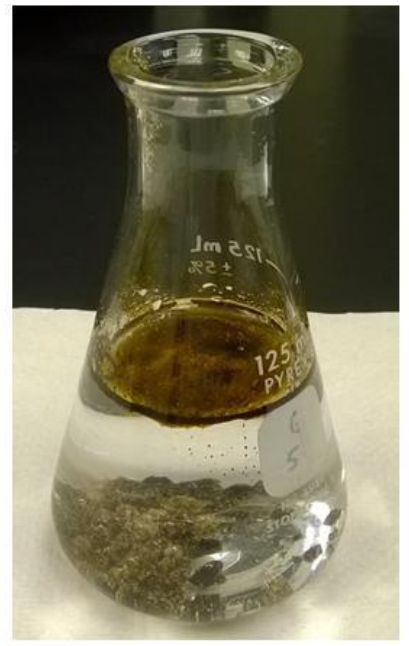

b

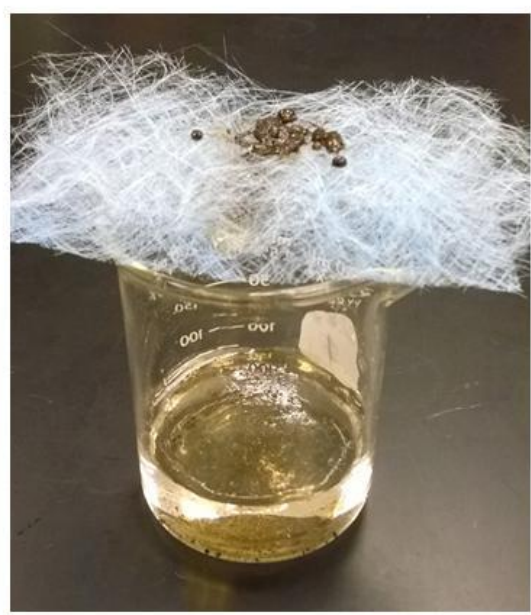

C

Figure 2.4. Formation of oil-sand aggregates: a. Floating oil; b. Formation and separation of particle-oil aggregates; and c. Particle-oil aggregates separated by coarse filtration (crude oil-fine quartz aggregates). 


\section{Results}

Figure 2.5 compares the formation of the aggregate phase with increasing amount of granular material added to the salt water solution containing $0.9 \mathrm{~g}$ of crude oil. There was a significant reduction in the amount of floating oil with increasing amount of granular material added.

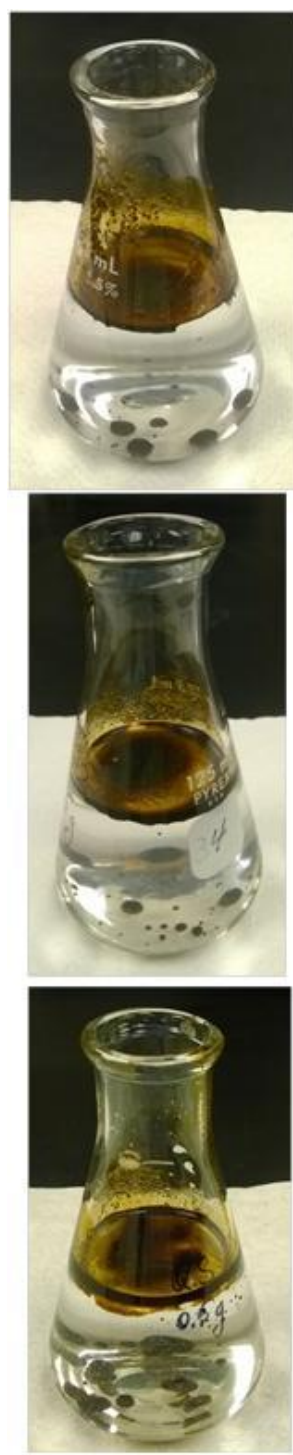

$$
0.5 \mathrm{~g}
$$
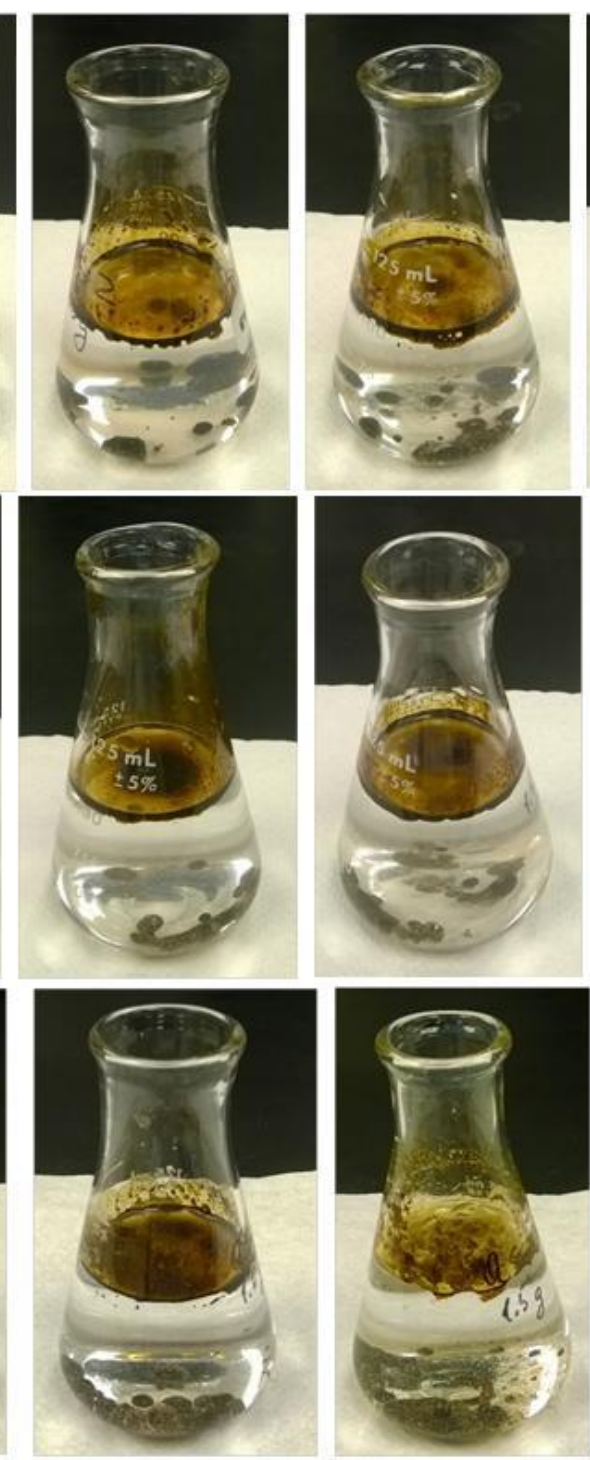

$1.5 \mathrm{~g}$
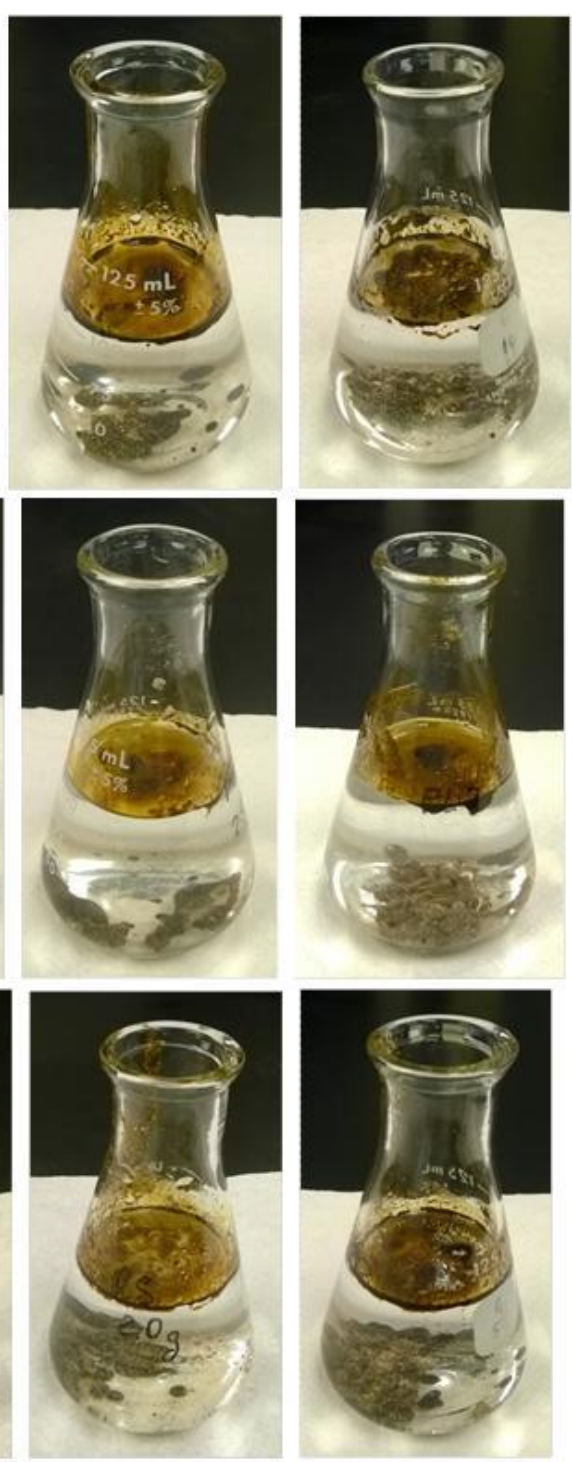

$2.0 \mathrm{~g}$

$3.5 \mathrm{~g}$

Figure 2.5. Comparison of oil-particle aggregate formation with different granular particles with increasing particle/oil ratios. Top row: medium limestone; middle row: fine limestone; and bottom row: fine quartz. 
Table 2.3 summarizes the removal efficiency of the granular materials for capturing the floating oil during the experiments. Oil removal efficiency increased with increasing amount of granular materials applied. However, the amount of oil removed from the floating phase did not increase linearly with the amount of granular materials added as presented in Fig. 2.6a. The oil capture efficiency increased significantly when the amount of granular material added was between 1 and 2 grams corresponding to granular material to oil ratios between approximately 1 and $2 \mathrm{~g} / \mathrm{g}$ (note that specific gravity of crude oil is about 0.9) (Fig. 2.6b).

Figure 2.7 presents the oil to granular material content of the aggregates separated in relation to granular materials to floating oil ratio. It is interesting to note that the aggregates separated had higher amounts of oil content when small amounts of granular material were applied. 

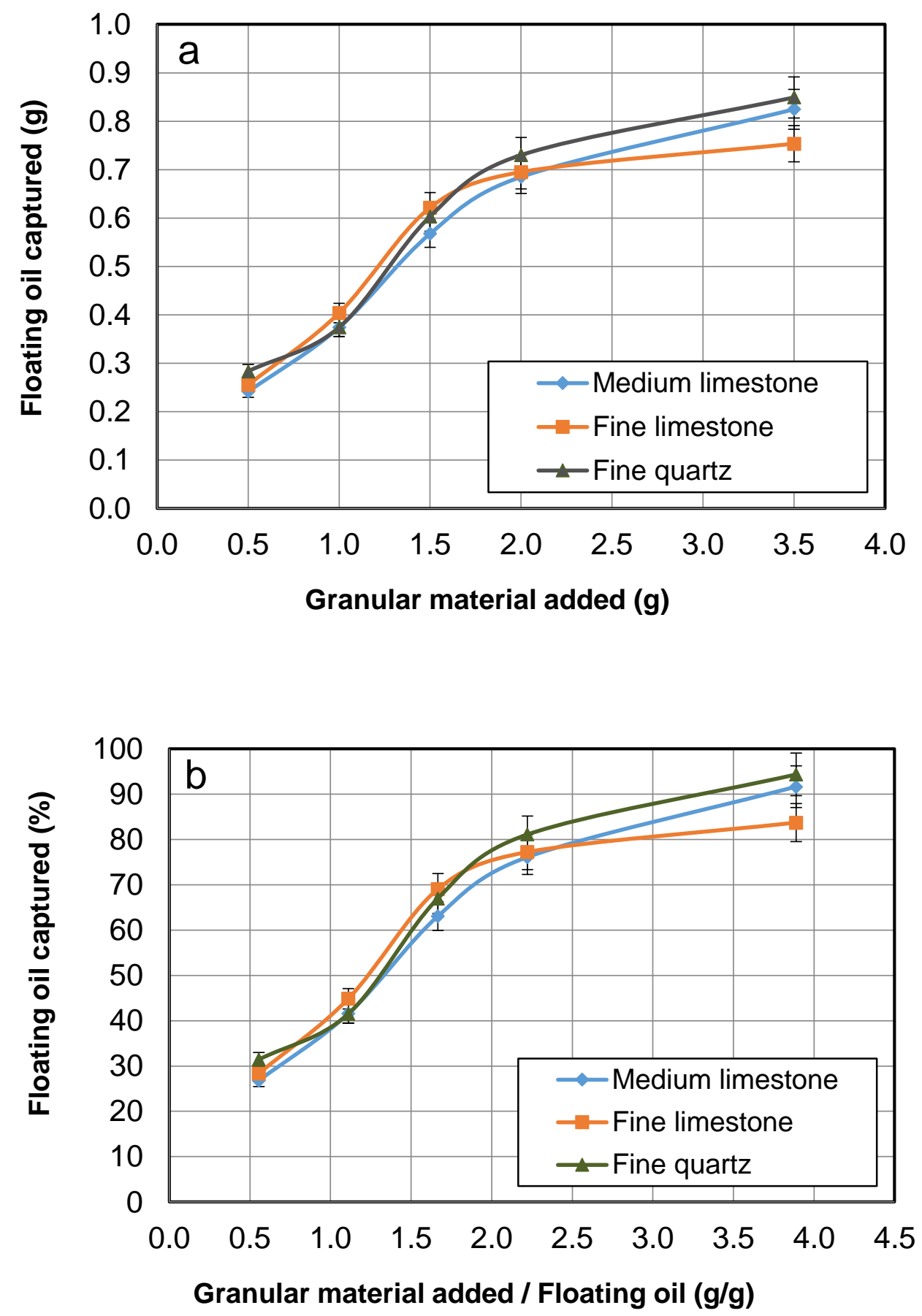

Figure 2.6. Capture of floating oil by granular materials: a. Change in the amount of oil captured in the aggregate phase in relation to amount of granular material added; and $\mathbf{b}$. Oil removal efficiency in relation to the ratio of granular material added to floating oil present. 


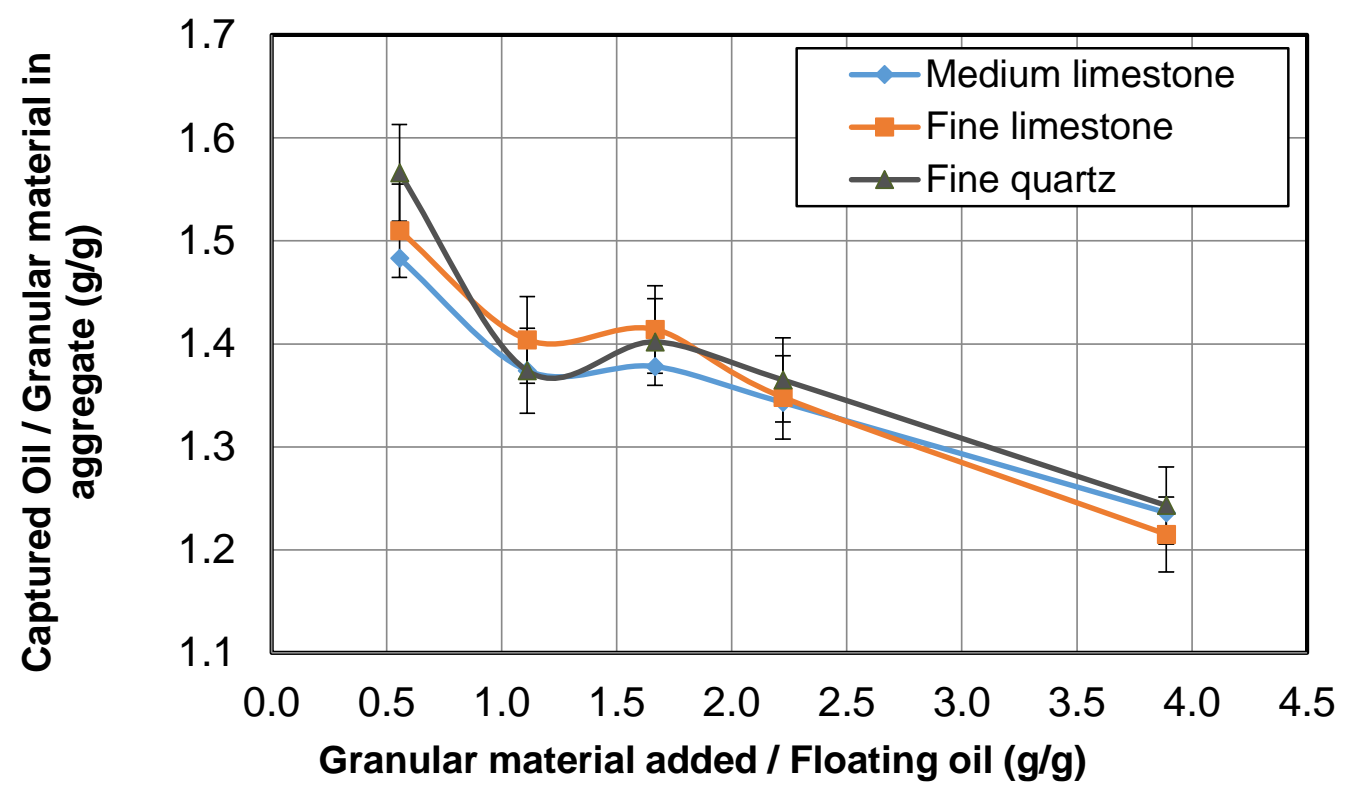

Figure 2.7. Variation oil capture coefficient $(k)$ of the granular aggregates in relation to the ratio of granular material added to floating oil present.

Table 2.3. Oil capture efficiency $(E)$ of granular materials used.

\begin{tabular}{lccccc}
\hline & \multicolumn{5}{c}{ Amount of granular particles added (g) } \\
\cline { 2 - 6 } Material & 0.5 & \multicolumn{1}{c}{1.0} & 1.5 & 2.0 & 3.5 \\
\cline { 2 - 6 } & \multicolumn{5}{c}{ Oil removal efficiency $(\%$ w/w) } \\
\hline $\begin{array}{l}\text { Medium limestone } \\
\text { Average }\end{array}$ & 26.81 & 41.56 & 63.06 & 76.11 & 91.64 \\
St. Dev. & 20.34 & 11.05 & 21.71 & 20.40 & 10.15 \\
\hline Fine limestone & & & & 77.22 & 83.72 \\
Average & 28.33 & 44.86 & 69.03 & 14.37 & 7.26 \\
St. Dev. & 8.91 & 18.01 & 21.06 & \\
\hline Fine quartz & 31.44 & 41.53 & 67.00 & 81.11 & 94.35 \\
Average & 8.05 & 18.07 & 19.33 & 16.62 & 8.67 \\
St. Dev. & & & & \\
\end{tabular}

For the practical application of this method, the ratio of oil-particle aggregates forming (i.e., beach sand or limestone) to be used at a spill location can be estimated from 
the material balance (i.e., equation 5). Based on the experimental data presented in Table 2.3, the oil capture coefficient of the granular aggregates, $k$, can be defined as:

$$
k=\frac{M_{a}}{M_{p}}
$$

where $M_{a}$ is the mass of oil in the aggregate phase (i.e., filtered aggregate phase) (g); and $M_{p}$ is the mass of applied granular particles. Table 2.4 presents the oil capture coefficient of the granular aggregates.

Table 2.4. Experimental $k$ values (ratio of mass of oil-particle aggregates formed to mass of granular material added).

\begin{tabular}{lccccr}
\hline & \multicolumn{5}{c}{ Amount of granular material added $(\mathrm{g})$} \\
\cline { 2 - 6 } Material & 0.5 & \multicolumn{1}{c}{1.0} & 1.5 & 2.0 & 3.5 \\
\cline { 2 - 6 } & \multicolumn{5}{c}{ Oil-granular material ratio in aggregates $(\mathrm{g} / \mathrm{g})$} \\
\hline $\begin{array}{l}\text { Medium } \\
\text { limestone, } k_{M L}\end{array}$ & 1.48 & 1.37 & 1.38 & 1.34 & 1.24 \\
$\begin{array}{l}\text { Fine limestone, } \\
k_{F L}\end{array}$ & 1.51 & 1.40 & 1.41 & 1.35 & 1.22 \\
Fine quartz, $k_{F Q}$ & 1.57 & 1.37 & 1.40 & 1.37 & 1.39 \\
\hline
\end{tabular}

Table 2.4 presents $k$ values, formula (9), for three series of experiments. Figure 2.7 presents the oil/solid ratio in the aggregates ( $k$ values) in relation to solid/oil ratio added. The amount of oil captured in the aggregate phase does not increase with increasing amount of solids added when the oil/sand ratio is between 0.6 and 1.0.

To estimate the amount of solid particles to be added to capture an oil spill with a total mass of $M_{T o}$, the equation (8) can modified as:

$$
M_{p}=\frac{E M_{o}}{100(k-1)} \cdot \frac{M_{T o}}{M_{o}}=\frac{E M_{T o}}{100(k-1)}
$$


For example, the amount of granular material needed for a desired capture efficiency of $90 \%$ for 1000 barrels of floating oil $\left(1 \mathrm{barrel}=120 \mathrm{~L} ; 1000\right.$ barrel $=120 \mathrm{~m}^{3}$, for oil density $900 \mathrm{~kg} / \mathrm{m}^{3} M_{T o}=10^{5} \mathrm{~kg}$ ), would be (using average $k$ value of 1.38):

$$
M_{p}=\frac{E M_{T o}}{100(k-1)}=\frac{90 \cdot 10^{5}}{100(1.38-1)}=2.37 \cdot 10^{5} \mathrm{~kg}\left(\text { or } 5.2210^{5} \mathrm{lbs}\right) .
$$

This result does not correlate well with the amount of sand required to encapsulate $1 \mathrm{~L}$ of oil that was calculated by Abkarian et al. (2013), and was equal to $0.1-0.5 \mathrm{~kg}$. The difference is attributed to variations in particle sizes and density and may also be explained by the fact that sand particles do not only cover an oil droplet on the surface but are absorbed into the oil droplets. There is also loss of particles that do not hit the oil surface and settle without aggregation.

\section{Conclusions}

Experiments were conducted with natural granular materials to aggregate floating crude oil. Limestone and sand applications were effective in capturing the floating oils. The results showed that small amounts of granular particles can capture and form stable aggregates with crude oil which settled very quickly. The natural granular materials can be used for controlling the transport of floating oils with surface current and water-air interactions especially near coastal areas where dispersant application may not be suitable. About $50 \%$ of the floating oil was removed by aggregates regardless of the material used when granular material to floating oil ratio was about $1 \mathrm{~g} / \mathrm{g}$. The aggregates had higher oil content when the granular material applied was $0.5 \mathrm{~g} / \mathrm{g}$. 


\section{Acknowledgments}

Partial funding for this research has been provided by Gulf of Mexico Research

Initiative (GoMRI) through funding to Consortium for the Molecular Engineering of Dispersant Systems (C-MEDS).

\section{References}

Abkarian, M., Protiere, S., Aristoff, J.M., and Stone, H.A. 2013. Gravity-induced encapsulation of liquids by destabilization of granular rafts. Nature Communications, 4 (1895).

Bhushan, B. 2003. Adhesion and stiction: mechanisms, measurement techniques, and methods for reduction. Journal of Vacuum Science \& Technology B, 21 (6): 22622296.

EPA/600/R-03/072. USEPA. 2003. Characteristics of spilled oils, fuels, and petroleum products: 1. Composition and properties of selected oils.

Fingerle, A., and Herminghaus, S. 2008. Equation of state of wet granular matter. Physical Review, E 77, 011306.

Herminghaus, S. 2005. Dynamics of wet granular matter. Advances in Physics, 54: 221261.

Iverson, R.M., and Vallance, J.W. 2001. New views of granular mass flows. Geology, 29 (2): 115-118.

Kaku, V.K., Boufadel, M.C., Venosa, A., and Weaver, J. 2006. Flow dynamics in eccentrically rotating flasks used for dispersant effectiveness testing. Environmental Fluid Mechanics, 6: 385-406.

Kusters, K.A., Wijers, J.G., and Thoenes, D. 1997. Aggregation kinetics of small particles in agitated vessels. Chemical Engineering Science, 52 (1): 107-121.

Lehr, W., Jones, R., Evans, M., Simecek-Beatty, D., and Overstree, R. 2002. Revisions of the ADIOS oil spill model. Environmental Modeling and Software, 17: 191-199.

Manger, G.E. 1963. Porosity and bulk density of sedimentary rocks. Geological Survey Bulletin, 1144-E. 
Mitarai, N., and Nori, F. 2006. Wet granular materials. Advances in Physics, 55 (1-2): 145.

Payne, J.R., Kirstein, B.E., Clayton, J.R., Clary, C., Redding, R., McNabb, D., and Farmer, G. 1987. Integration of suspended particulate matter and oil transportation study. Final report. Minerals Management Service, Alaska.

Rossetti, D., Pepin, X., and Simons, S.J.R. 2003. Rupture energy and wetting behavior of pendular liquid bridges in relation to the spherical agglomeration process. Journal of Colloid and Interface Science, 261: 161-169.

Simons, S.J.R. 1996. Modelling of agglomerating systems: from spheres to fractals. Powder Technology, 87: 29-41. 


\title{
CHAPTER III
}

\section{PARTITIONING OF FRESH CRUDE OIL BETWEEN FLOATING, DISPERSED AND SEDIMENT PHASES: EFFECT OF EXPOSURE ORDER TO DISPERSANT \\ AND GRANULAR MATERIALS}

Boglaienko, D., and Tansel, B. 2016. Journal of Environmental Management, 175: 40-45.

DOI: 10.1016/j.jenvman.2016.03.017

\begin{abstract}
When three or more high and low energy substrates are mixed, wetting order can significantly affect the behavior of the mixture. We analyzed the phase distribution of fresh floating Louisiana crude oil into dispersed, settled and floating phases depending on the exposure sequence to Corexit 9500A (dispersant) and granular materials. In the experiments artificial sea water at salinity $34 \%$ was used. Limestone (0.30 to $2.00 \mathrm{~mm})$ and quartz sand $(0.075$ to $0.300 \mathrm{~mm})$ were used as the natural granular materials. Dispersant Corexit 9500A increased the amount of dispersed oil up to $33.76 \pm 7.04 \%$. Addition of granular materials after the dispersant increased dispersion of oil to $47.96 \pm$ $1.96 \%$. When solid particles were applied on the floating oil before the dispersant, oil was captured as oil-particles aggregates and removed from the floating layer. However, dispersant addition led to partial release of the captured oil, removing it from the aggregated form to the dispersed and floating phases. There was no visible oil aggregation with the granular materials when quartz or limestone was at the bottom of a flask before the addition of oil and dispersant.
\end{abstract}




\section{Introduction}

Dispersion of floating crude oil is one of the main processes linked to spilled oil degradation in the marine environment. This process has been extensively and intensively studied, resulting in development of chemical dispersants and their implementation in oil spill treatment practice.

Besides mechanical dispersion (wave turbulence), chemical dispersion (surfactants, or dispersants), interaction of oil with mineral particles and sediments has been recognized as an important factor in oil spill dispersion. Dispersed oil-mineral aggregates may eventually settle, trapping oil on the bottom (Lee, 2002; Sterling et al., 2004; Gong et al., 2014). Moreover, fine sediments on shorelines add to natural oil removal (surf-washing) through formation of oil-mineral aggregates (Jahns et al., 1991; Bragg and Owens, 1995; Stoffyn-Egli and Lee, 2002). A method that implies dispersion of mineral solid particles in aqueous phase for oil removal via buoyant mineral solids-oil floccules was patented (Bragg and Yang, 1996). Direct application of granular materials on the floating oil surface, followed by immediate oil sedimentation and stabilization, has been recently studied and identified as a potential oil spill remediation method (Abkarian et al., 2012; Boglaienko and Tansel, 2015).

Gordon et al. (1973) reported that adsorption of oil on the surface of suspended particulate matter may result in removal of oil from the water surface by $87-98 \%$. Later, Muschenheim and Lee (2002) reviewed and discussed the role of additional to adsorption processes that influence surface oil removal by particulate matter, including flocculation of fine particles with surface oil. Passow et al. (2012) pointed out marine snow formation in the Gulf of Mexico after the Deepwater Horizon accident and the role of coagulation of 
oily particulate matter. In general, two main oil-particle interactions have been specified: adsorption of hydrocarbons and direct aggregation of oil with particulate matter (Gong et al., 2014; Bandara et al., 2011; Lee, 2002). A numerical model that simulates oil and suspended sediment interaction, and estimates the fraction of removed oil was presented by Bandara et al. (2011), according to which the amount of removed oil as oil-sediment aggregates can reach up to $65 \%$.

Colloidal solid particles (clay) can attach on oil droplet surfaces (Dommersnes et al., 2013). At high clay concentrations (above about $2 \% \mathrm{w} / \mathrm{w}$ ), the droplet surface can be covered by solid particles. Chemical dispersants decrease the oil-particle interaction. Physically dispersed oil (i.e., emulsion formed by mixing) associates easily with particulate matter in comparison to the chemically dispersed oil (i.e., using dispersants) (Mackay and Hossain, 1982; Payne et al., 2003). Similarly, another study showed that addition of a dispersant decreased the adsorption of oil to suspended particulate matter (Sørensen et al., 2014). Dispersant Corexit 9500A contains anionic surfactants, nonionic surfactants, and organic solvents (Nalco, 2014). Such nonionic components of the Corexit dispersants as Tween 80 adsorbs to oil droplets irreversibly, resulting in long coalescence time (Reichert and Walker, 2015).

High energy substrates are more easily wet than low energy substrates (De Gennes, 1985). Also the wetting process is more complete when the substrate has a relatively higher surface energy than the liquid (Kern et al., 1986). Addition of a surfactant to water decreases wetting and adhesion work of high energy polar solid materials (i.e., quartz) (Zdziennicka and Jańczuk, 2010). A dispersant, designed to lower surface tension (surface free energy), contributes to smaller rates of oil-mineral aggregation, as it becomes less 
beneficial (i.e., smaller decrease in free energy) for the system to go from "oil and solids in water" to "oil-solid aggregates in water."

$$
\Delta G=\gamma_{s o}-\gamma_{w o}-\gamma_{s w}
$$

where $\gamma$ is surface free energy $\left(\gamma_{i}=\partial G / \partial A_{i}, A\right.$ is area); subscripts: $s$ is solids, $o$ is oil, and $w$ is water.

For the system to experience decrease in free energy for oil-solids aggregation (i.e., $\Delta G \leq 0)$

$$
\gamma_{s o} \leq \gamma_{w o}+\gamma_{s w}
$$

Addition of the dispersant decreases $\gamma_{w o}$, so that condition (2) may not be satisfied.

The change in surface free energy, $\Delta G$, can be described in two steps: $1 . \Delta G_{1}$ for activation of energy barrier to the adhesion of solid particle to oil, and 2. $\Delta G_{2}$ for work of adhesion, $w_{a}$ (Adamson, 1990). These steps can be expressed as follows:

$$
\Delta G=\Delta G_{1}+\Delta G_{2}
$$

where,

$$
\begin{aligned}
& \Delta G_{1}=\gamma_{w o} \Delta A_{w o} \\
& \Delta G_{2}=\left(\gamma_{s o}-\gamma_{w o}-\gamma_{s w}\right) A_{s o}=-w_{a} A_{s o} \\
& \Delta G=\gamma_{w o} \Delta A_{w o}-w_{a} A_{s o}
\end{aligned}
$$

Surface energy plays an important role in wetting phenomena which can affect the phase distribution of oil. When three or more materials with significantly different surface energies are mixed, the order of mixing of the materials can significantly alter the phase distribution of oil. In this study we analyzed the phase distribution of crude oil, as floating oil, dispersed oil, and oil captured by solid granular materials, depending on their presence and absence, and the order of exposure of the floating oil to granular materials and 
dispersant Corexit 9500A. The experiments aimed to quantify the partitioning of the floating crude oil and the effectiveness of the dispersant in relation to the exposure order of crude oil to granular materials and Corexit 9500. In the experiments, sand (quartz) and limestone were used as the granular materials. South Louisiana crude oil was chosen due to the fact that it was spilled during the Deepwater Horizon oil spill (also referred to as the BP oil spill, the BP oil disaster, and the Gulf of Mexico oil spill in 2010). The partitioning of crude oil on granular surfaces and in the water phase was interpreted in view of the changes in surface energy with exposure order.

\section{Materials and Methods}

\section{Materials}

Louisiana crude oil (Mississippi Canyon Block 252) was supplied by BP America Production Company in Houston, Texas, USA. The Louisiana crude oil has American Petroleum Institute (API) gravity of 36.2 at $15^{\circ} \mathrm{C}$, which is classified as a light crude oil. The dispersant Corexit 9500A used in this study was supplied by Exxon/Nalco Energy Chemicals, Sugar Land, Texas, USA. Limestone of medium particle size (0.30 to $2.00 \mathrm{~mm})$ and quartz sand of fine particle size $(0.075$ to $0.300 \mathrm{~mm})$ were used as the natural granular materials (GM). Instant Ocean Sea Salt (Aquarium System, Mentor, Ohio, USA) and deionized water were used to prepare the stock solutions of artificial sea water at salinity level of $34 \%$. The solvent dichloromethane (DCM), pesticide-quality, was used for the oil extraction and preparation of standards. 


\section{Experimental procedure}

Six series of experiments were conducted as designated by ODS, OSD, SOD, OS, $\mathrm{OD}$ and $\mathrm{O}$ indicating materials present and the order of adding the materials (Fig. 3.1). For the experimental design, $\mathrm{O}$ is crude oil, $\mathrm{D}$ is dispersant, and $\mathrm{S}$ is solid granular material.

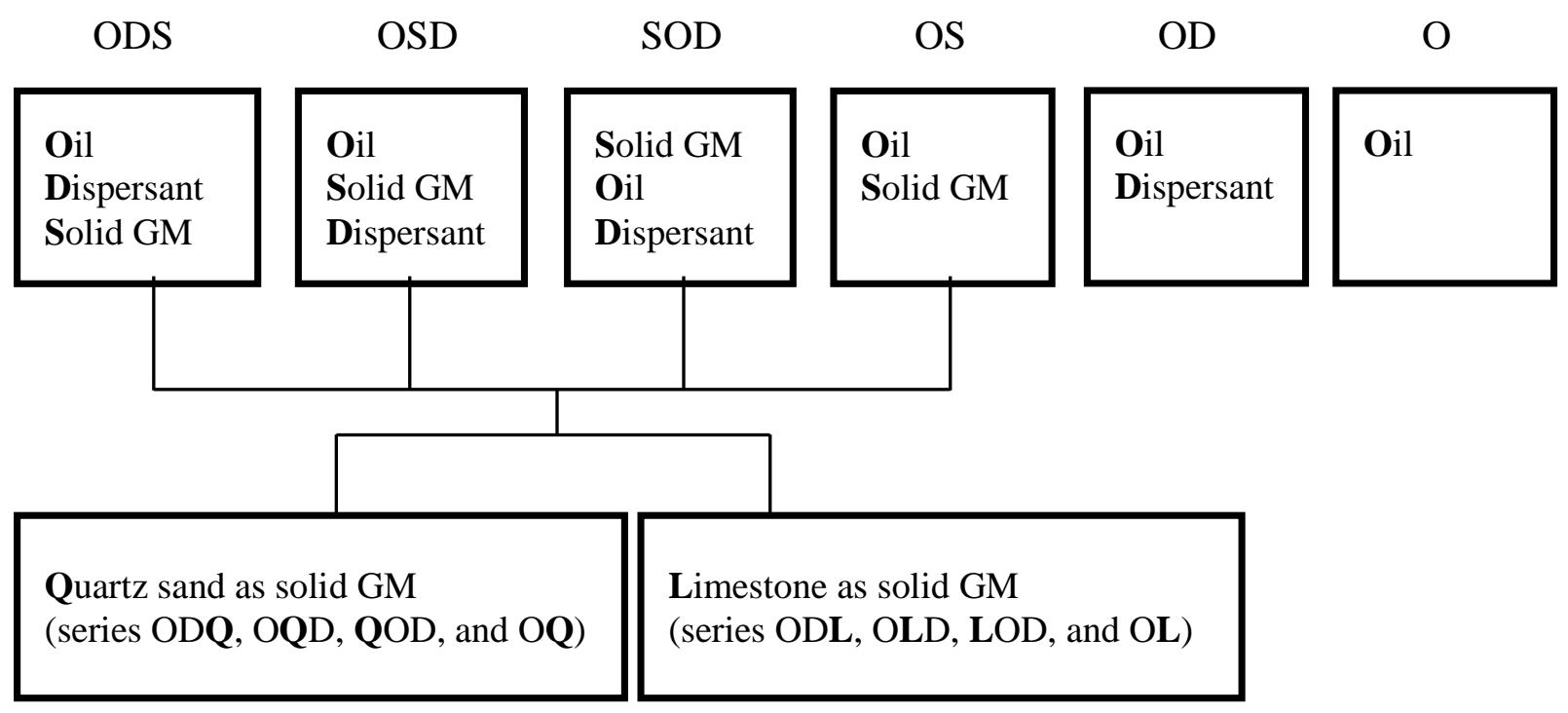

Figure 3.1. Experimental design (O: crude oil, D: dispersant, S: Solid granular material, GM: granular material).

A volume of $0.5 \mathrm{~mL}$ fresh Louisiana crude oil was added to $100 \mathrm{~mL}$ saltwater in a baffled flask. Dispersant Corexit 9500A was used at the dispersant-to-oil ratio (DOR) 1:25 $(0.02 \mathrm{~mL})$. All series had the same amount of granular material $(1 \mathrm{~g})$ but varied by the order of its application. Series ODS had granular material added to the mixture (from surface) after the dispersant; series OSD had the dispersant applied on the mixture surface after the oil aggregation by granular materials; series SOD had the granular materials on the bottom of a flask, followed by the addition of oil and dispersant; for the OS series no dispersant was used and the oil removal process was considered by the granular materials and the aggregation of particles as they pass through the floating phase; OD series had oil removed 
(i.e., dispersed) by Corexit 9500A only; and the $\mathrm{O}$ series had only oil in the salt water mixture. All experiments were conducted in triplicate for limestone and quartz sand at room temperature $\left(20-22{ }^{\circ} \mathrm{C}\right)$. Flasks were placed on the orbital shaker and mixed at 200 rpm for 10 min (to simulate mixing conditions in the environment). After stopping the shaker, the flasks were left for $10 \mathrm{~min}$ to settle the granular particles.

An extraction procedure, similar to the Swirling flask test (U.S. EPA, 1997), was performed to estimate the amount of the dispersed oil. Extraction utilized pesticide-quality DCM and $30 \mathrm{~mL}$ of the aqueous mixture containing the dispersed oil. DCM was added to the mixture in the amount of $5 \mathrm{~mL}$ three times, shaken and drained from the separatory funnel. Standard solutions of oil and two series of standard solutions of oil and dispersant were prepared following the EPA SFT guidelines (U.S. EPA, 1997). The UV-visible spectrophotometer was calibrated at the wavelengths 400 and $420 \mathrm{~nm}$, as better precision was obtained for our samples. Total mass of the dispersed oil was determined from the mean of the measured levels at the calibrated wavelengths.

Samples with solid material were filtered through the fiberglass filter to estimate the amount (by weight) of oil captured by the granular materials. The floating oil was discarded before filtering. The amount of floating oil remaining was calculated based on the amounts of oil in the dispersed phase (determined by extraction) and the oil captured by the granular materials (determined gravimetrically). Dispersion effectiveness (DE) was calculated as percent of the dispersed phase (mass of oil found by the extraction procedure) in relation to the initial mass of the crude oil. Statistical analyses were performed on the DE differences between the series using two-way ANOVA test (IBM SPSS 21). 


\section{Results and Discussion}

Phase distribution of oil in relation to the order of the dispersant and solid granular material applications is summarized in Fig. 3.2a for quartz and Fig. 3.2b for limestone as granular material. The amount of oil dispersed in salt water by the means of shaking only ('O' series) was $1.47 \pm 0.58 \%$ and the rest was floating on surface (Fig. 3.3). Dispersant Corexit 9500A increased the amount of dispersed oil up to $33.76 \pm 7.04 \%$ and the rest was in the floating phase ('OD' series), Fig. 3.2a,b and Fig. 3.3. Addition of fine quartz particles after the dispersant ('ODQ' series) resulted in increased dispersion of oil, $47.96 \pm 1.96 \%$, Fig. 3.2a,b. Dispersion of oil in cases when medium limestone particles were added ('ODL' series) was not affected, as compared to 'OD' mixture, $33.77 \pm 16.27 \%$. In both cases (with quartz and limestone) settled particles contained a negligible amount of oil as 'captured' phase, Fig. 3.4. There was no visible captured oil as well when quartz or limestone was at the bottom of a flask before addition of oil and dispersant application ('QOD' and 'LOD' series), Fig. 3.2a,b and Fig. 3.4; the amount of the dispersed oil with the presence of quartz was $28.36 \pm 5.87 \%$ and with the presence of limestone it was 24.84 $\pm 8.16 \%$; the floating phase had less oil for the quartz than for limestone series. When solid particles were applied on the floating oil before the dispersant ('OQD' and 'OLD'), oil was captured as oil-particle aggregates and removed from the floating layer. However, dispersant addition to the flasks led to partial release of the captured oil, moving it from the aggregated form to the dispersed and floating phases, Fig. 3.2a,b, Fig. 3.3, and Fig. 3.4. The amount of dispersed oil resulted in $13.11 \pm 1.37 \%$ for quartz and $19.51 \pm 4.01 \%$ for limestone. Finally, when solid granular material was utilized with no dispersant afterwards, most of the oil was aggregated, settled and stayed as captured phase (oil-particle globules) 
even at the end of the shaking period ('OQ' and 'OL' series) (Fig. 3.3 and Fig. 3.4). The amount of dispersed oil was $0.69 \pm 0.26 \%$ and $0.79 \pm 0.13 \%$.

The two-way ANOVA test was performed to evaluate whether there was significant difference between quartz and limestone, and between different series that utilized solid granular material $(\alpha=0.05)$. The dependent variable was set as 'DE' (Dispersion Effectiveness). The first factor level was 'Series' (4 types) and the second factor level was 'GM' (Granular Material, 2 types) with no control of the block effect (no interaction between factor levels, interaction tested $p$-value $=0.243$ ). Assumptions of equal variances and normality were tested and satisfied (Levene's test's $p$-value $=0.327$ for DE via GM factor and $p$-value $=0.047$ for $\mathrm{DE}$ via Series factor that has marginal significance at the 0.05 level. Shapiro test's $p$-value $=0.746$ ). The two-way ANOVA test showed that there was no significant difference between limestone or quartz sand $(p$-value $=0.448$ ) but there was significant difference between series (i.e., order of materials added) ( $p$-value $=2.29 \mathrm{E}$ 06). Tukey HSD post hoc test indicated significant difference between all pairs except ODS and SOD, and SOD and OSD (Fig. 3.5).

The results showed that when three or more high and low energy substrates were mixed, wetting order significantly affected the behavior of the mixture. When granular materials were introduced to the oil phase (floating on water), oil and granular particles (high surface energy) had adequate interaction and contact time to form strong noncovalent bonds.

However, when the granular materials were introduced to the water first, their surface energy was significantly reduced by wetting with water, and hence, were unable to have effective interaction with oil. Dispersant reduced the surface energy of both the oil 
and water. Hence, the affinity of oil to form noncovalent interactions with granular particles (with high surface energy) was significantly reduced in comparison to that of water. As a result, wetting of granular particles with water occurred, further reducing the ability of dispersed oil to attach on solid particle surfaces.

From the standpoint of toxicity, the dispersed fraction contains higher concentrations of polycyclic aromatic hydrocarbons (PAH) than the water fraction without the dispersant, as it influences dissolution of PAH from the crude oil to the water column (Couillard et al., 2005; Griffin and Calder, 1977). The water soluble fraction of oil is expected to be in the dispersed phase and additional studies are needed to investigate distribution and partitioning of oil components to the granular particles. 

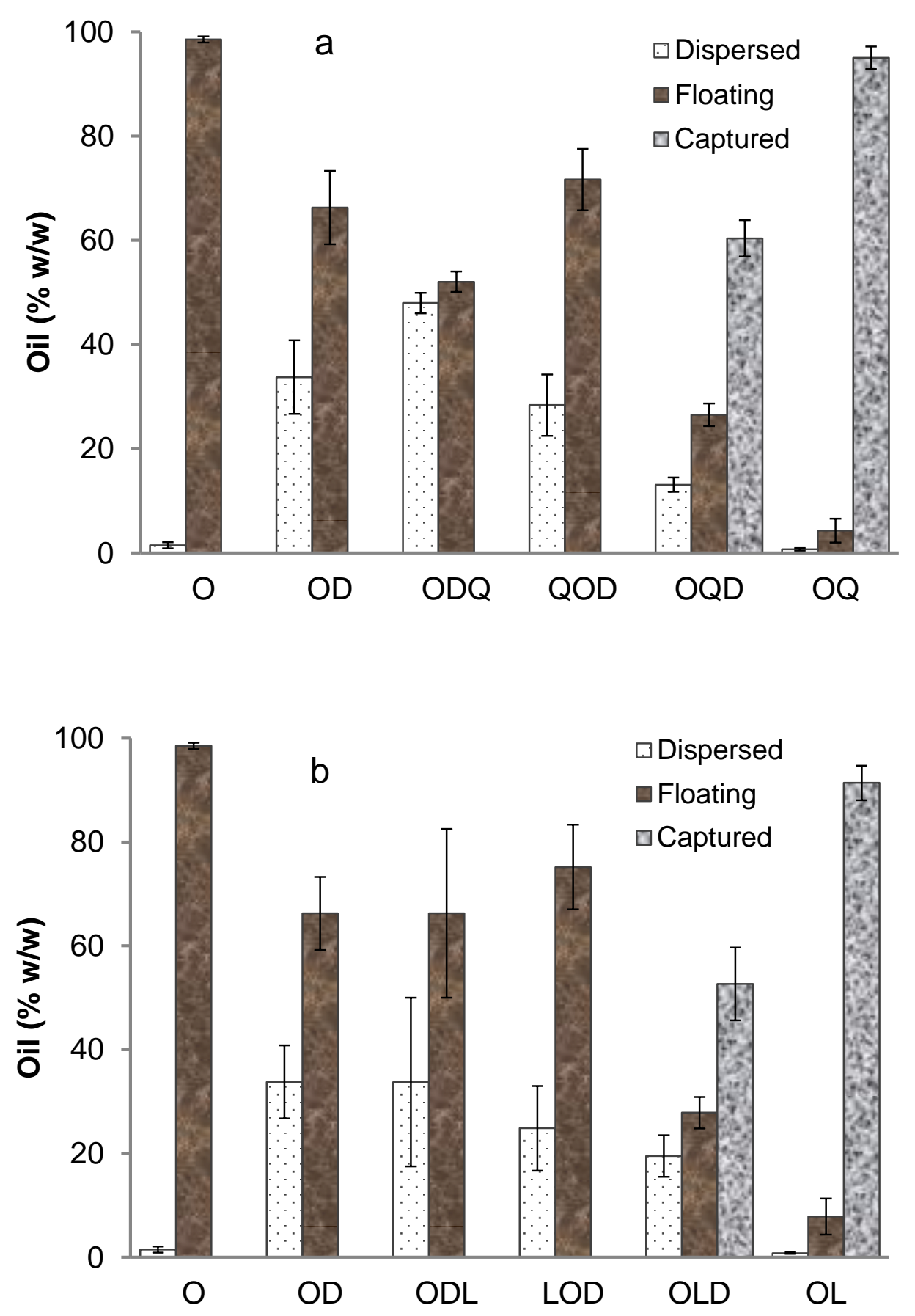

Figure 3.2. Phase distribution of oil depending on the presence and absence of the dispersant Corexit 9500A and granular materials (error bars: $\pm 1 \mathrm{SD}$ ): a. Fine quartz used as solid granular material; and $\mathbf{b}$. Medium limestone used as solid granular material. 
Table 3.1. Partitioning of floating oil between float and sediment phases relative to concentration in water phase.

\begin{tabular}{lcc}
\hline \multirow{1}{*}{ Order of exposure } & \multicolumn{2}{c}{ Partition ratio } \\
\cline { 2 - 3 } & Float/Water (w/w) & $\begin{array}{c}\text { Sediment/Water } \\
(\mathrm{w} / \mathrm{w})\end{array}$ \\
\hline Oil only & $82.81 \pm 1.45$ & - \\
Oil + Dispersant & $2.62 \pm 0.51$ & - \\
Oil + Dispersant + Quartz & $1.09 \pm 0.25$ & 0 \\
Oil + Dispersant + Limestone & $2.62 \pm 0.21$ & 0 \\
Quartz + Oil + Dispersant & $2.68 \pm 0.21$ & 0 \\
Limestone + Oil + Dispersant & $3.55 \pm 0.32$ & $4.60 \pm 1.55$ \\
Oil + Quartz + Dispersant & $2.03 \pm 0.30$ & $2.70 \pm 0.52$ \\
Oil + Limestone + Dispersant & $1.45 \pm 0.22$ & $137.68 \pm 1.55$ \\
Oil + Quartz & $7.78 \pm 0.55$ & $115.17 \pm 1.33$ \\
Oil + Limestone & $11.04 \pm 1.45$ & \\
\hline
\end{tabular}



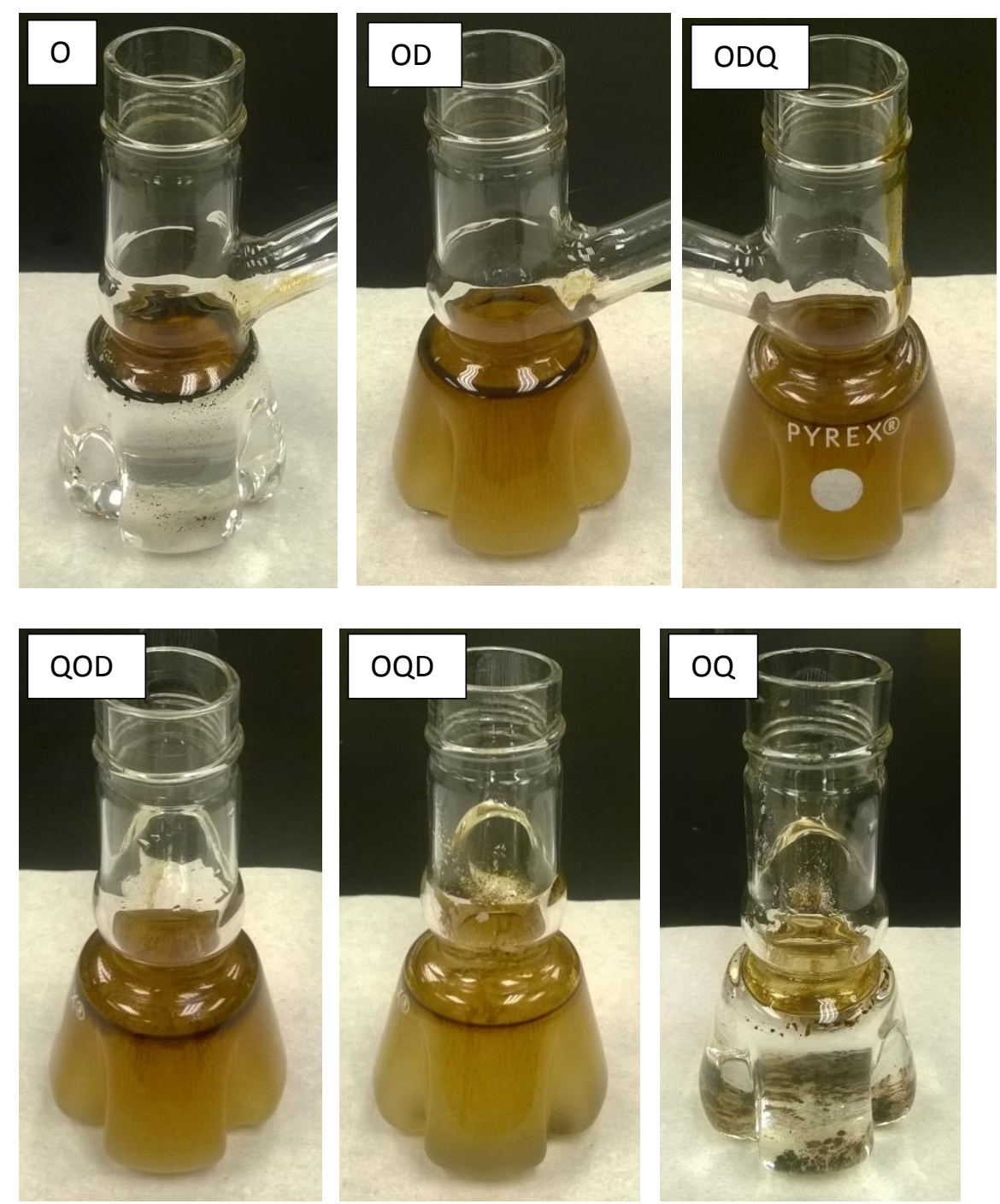

Figure 3.3. Profiles of mixtures illustrating the dispersed oil for different series. 

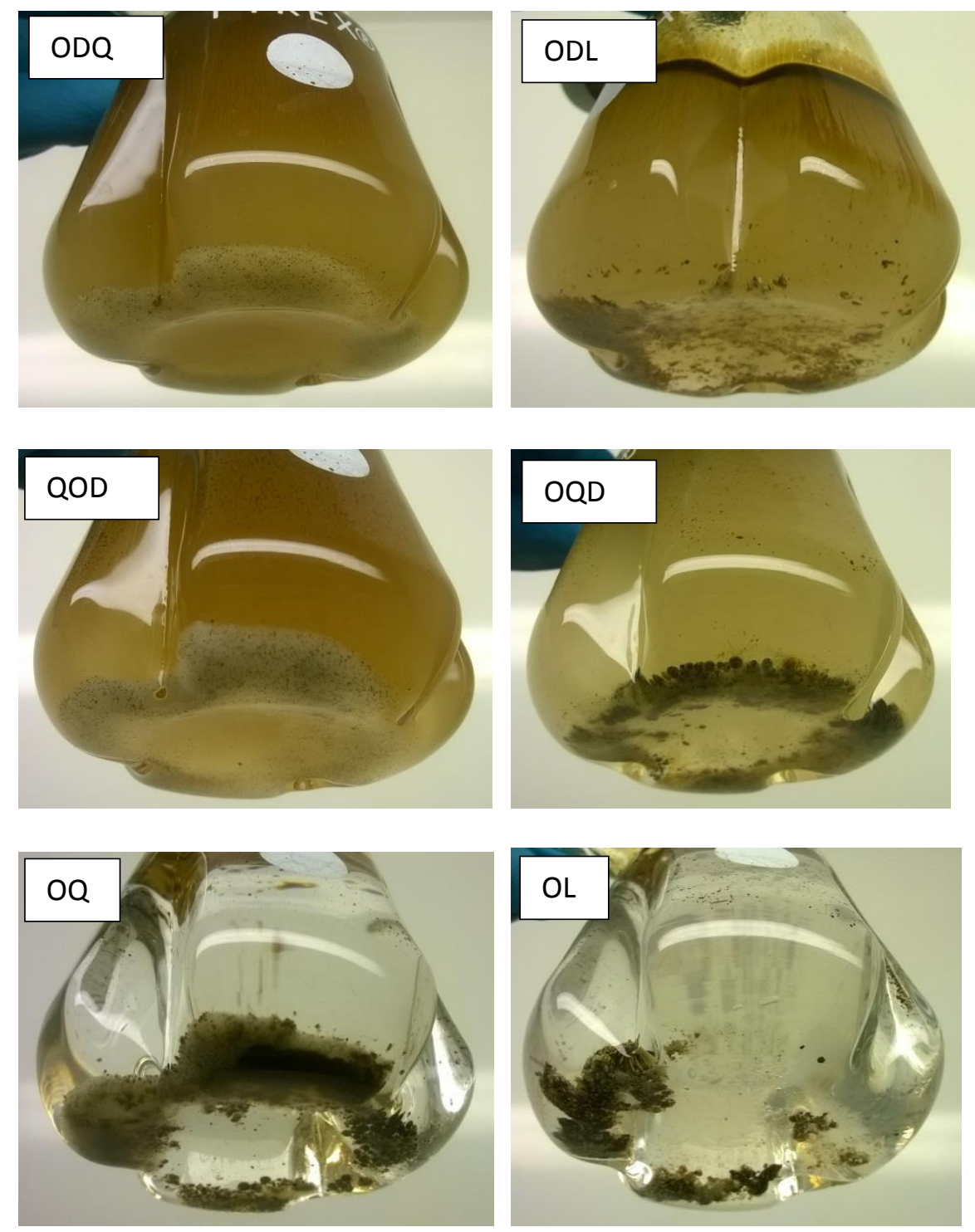

Figure 3.4. Bottom view of the mixtures illustrating oil capture by granular materials (as sediments) for different series. 


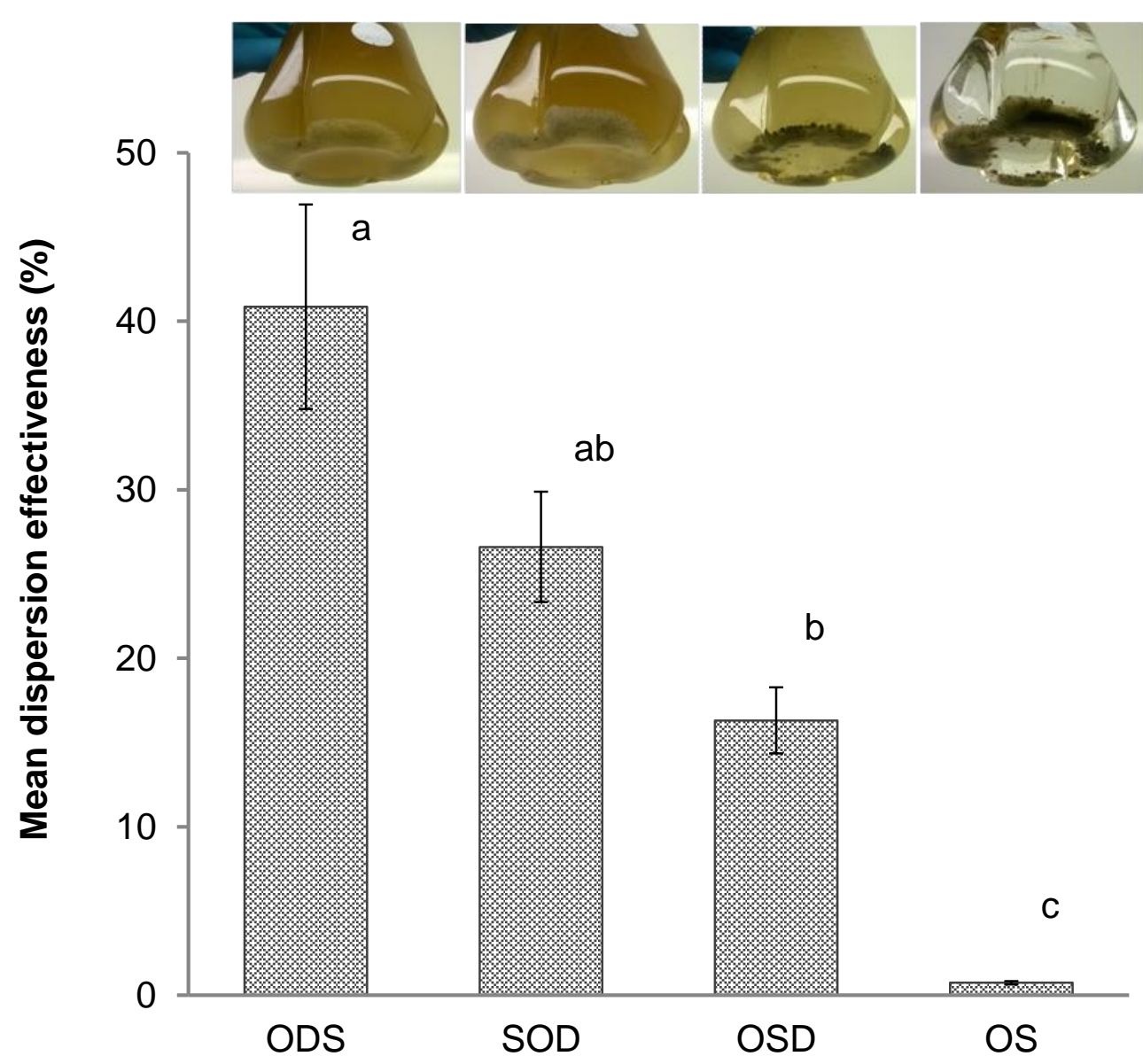

Figure 3.5. Dispersion effectiveness for different series that utilized granular materials; Tukey HSD post hoc $(p<0.05)$; error bars: \pm 1 SD. Significance: ODS-SOD $p=0.053$; ODS-OSD $p=0.001$; ODS-OS $p=0.000$; SOD-OSD $p=0.220$; SOD-OS $p=0.000$; OSDOS $p=0.032$ (Tukey HSD post hoc test).

\section{Conclusions}

Phase distribution of fresh floating Louisiana crude oil into dispersed, settled, and floating phases depends on the exposure order to Corexit 9500A dispersant and granular materials (limestone and quartz sand). Addition of the granular materials after the dispersant increased dispersion of oil from $33.76 \pm 7.04 \%$ (dispersant only) to $47.96 \pm$ $1.96 \%$. Oil can be efficiently captured and removed as oil-particles aggregates when no 
dispersant and only solid granular materials are utilized. Dispersant addition partially releases the captured oil, removing it from the aggregated form to the dispersed and floating phases. If quartz or limestone was present at the bottom of a flask before the addition of oil and dispersant, there was no visible oil aggregation with the granular materials.

\section{Acknowledgments}

Partial funding for this research has been provided by Gulf of Mexico Research Initiative (GoMRI) through funding to Consortium for the Molecular Engineering of Dispersant Systems (CMEDS).

\section{References}

Abkarian, M., Protiere, S., Aristoff, J.M., and Stone, H.A. 2013. Gravity-induced encapsulation of liquids by destabilization of granular rafts. Nature Communications, 4 (1895).

Adamson, A.W. 1990. Physical chemistry of surfaces. $5^{\text {th }}$ ed. New York: Wiley.

Bandara, U.C., Yapa, P.D., and Xie, H. 2011. Fate and transport of oil in sediment laden marine waters. Journal of Hydro-environment Research, 5 (3): 145-156.

Boglaienko, D., and Tansel, B. 2015. Instantaneous stabilization of floating oils by surface application of natural granular materials (beach sand and limestone). Marine Pollution Bulletin, 91 (1): 107-112.

Bragg, J.R., and Owens, E.H. 1995. Shoreline cleansing by interactions between oil and fine mineral particles. In Proceedings of the International Oil Spill Conference, Feb. 22-Mar 2, Long Beach, CA, American Petroleum Institute, Washington, DC, Publication No. 4620, pp. 219-227.

Bragg, J.R., and Yang, S.H. 1996. Method for forming mineral solids-oil floccules. U.S. Patent. US 5490940 A.

Couillard, C.M., Lee, K., Legare, B., and King, T.L. 2005. Effect of dispersant on the composition of the water-accommodated fraction of crude oil and its toxicity to larval marine fish. Environmental Toxicology and Chemistry, 24 (6): 1496-1504. 
De Gennes, P.G. 1985. Wetting: statics and dynamics. Reviews of Modern Physics, 57: 827-863.

Dommersnes, P., Rozynek, Z., Mikkelsen, A., Castberg, R., Kjerstad, K., Hersvik, K., and Fossum, J.O. 2013. Active structuring of colloidal armour on liquid drops. Nature Communications, 4 (2066).

Gong, Y., Zhao, X., Cai, Z., O'Reilly, S.E., Hao, X., and Zhao, D. 2014. A review of oil, dispersed oil and sediment interactions in the aquatic environment: influence on the fate, transport and remediation of oil spills. Marine Pollution Bulletin, 79 (1-2): 1633.

Gordon, D.C. Jr., Keizer, P.D., and Prouse, N.J. 1973. Laboratory studies of the accommodation of some crude and residual fuel oils in sea water. Journal of the Fisheries Research Board of Canada, 30 (11): 1611-1618.

Griffin, L.F., and Calder, J.A. 1977. Toxic effect of water-soluble fractions of crude, refined, and weathered oils on the growth of a marine bacterium. Applied and Environmental Microbiology, 33 (5): 1092-1096.

Jahns, H.O., Bragg, J.R., Dash, L.C., and Owens, E.H. 1991. Natural Cleaning of Shorelines Following the Exxon Valdez Spill. International Oil Spill Conference Proceedings: March 1991, Vol. 1991, No. 1, pp. 167-176.

Kern, K., David, R., Palmer, R.L., and Cosma, G. 1986. Complete wetting on 'Strong' substrates: Xe/Pt(111). Physical Review Letters, 56 (26): 2823-2826.

Lee, K. 2002. Oil-particle interactions in aquatic environments: influence on the transport, fate, effect and remediation of oil spills. Spill Science \& Technology Bulletin, 8 (1): 3-8.

Mackay, D., and Hossain, K. 1982. An exploratory study of naturally and chemically dispersed oil. Environmental Protection Service, Environment Canada EE-35, 124, Ottawa, Canada.

Muschenheim, D.K., and Lee, K. 2002. Removal of oil from the sea surface through particulate interactions: review and prospectus. Spill Science \& Technology Bulletin, 8 (1): 9-18.

Nalco. Corexit ingredients in Nalco [database online]. 2014. http://www.nalcoesllc.com/nes/1602.htm (accessed August 31, 2015).

Passow, U., Ziervogel, K., Asper, V., and Diercks, A. 2012. Marine snow formation in the aftermath of the Deepwater Horizon oil spill in the Gulf of Mexico. Environmental Research Letters, 7, 035301. 
Payne, R.J., Clayton, Jr. J.R., and Kirstein, B.E. 2003. Oil/Suspended particulate material interactions and sedimentation. Spill Science \& Technology Bulletin, 8 (2): 201221.

Reichert, M.D., and Walker, L.M. 2015. Coalescence behavior of oil droplets coated in irreversibly-adsorbed surfactant layers. Journal of Colloid and Interface Science, 449: 480-487.

Sørensen, L., Melbye, A.G., and Booth, A.M. 2014. Oil droplet interaction with suspended sediment in the seawater column: influence of physical parameters and chemical dispersants. Marine Pollution Bulletin, 78: 146-152.

Sterling, M.C., Bonner, J.S., Page, C.A., Fuller, C.B., Ernest, A.N.S., and Autenrieth, R.L. 2004. Modeling crude oil droplet-sediment aggregation in nearshore waters. Environmental Science \& Technology, 38 (17): 4627-4634.

Stoffyn-Egli, P., and Lee, K. 2002. Formation and characterization of oil-mineral aggregates. Spill Science \& Technology Bulletin, 8 (1): 31-44.

United States Environmental Protection Agency (U.S. EPA), 1997. Swirling Flask Dispersant Effectiveness Test, Revised Standard Dispersant Toxicity Test, and Bioremediation Agent Effectiveness Test. 40 CFR Ch. I (7-1-03 Edition), Pt. 300, App. C, 224-246.

Zdziennicka, A., and Jańczuk, B. 2010. Wettability of quartz in presence of nonionic surfactants and short chain alcohols mixtures. Journal of Colloid and Interface Science, 343: 594-601. 


\title{
CHAPTER IV
}

\section{GRANULAR ENCAPSULATION OF LIGHT HYDROPHOBIC LIQUIDS (LHL) \\ IN LHL-SALT WATER SYSTEMS: PARTICLE INDUCED DENSIFICATION \\ WITH QUARTZ SAND}

Boglaienko, D., Tansel, B., and Sukop, M.C. 2016. Chemosphere, 144: 1358-1364.

DOI: 10.1016/j.chemosphere.2015.10.019

\begin{abstract}
Addition of granular materials to floating crude oil slicks can be effective in capturing and densifying the floating hydrophobic phase, which settles by gravity. Interaction of light hydrophobic liquids (LHL) with quartz sand was investigated in LHLsalt water systems. The LHLs studied were decane, tetradecane, hexadecane, benzene, toluene, ethylbenzene, m-xylene, and 2-cholorotoluene. Experiments were conducted with fine quartz sand (passing sieve No. 40 with openings $0.425 \mathrm{~mm}$ ). Each LHL was dyed with few crystals of Sudan IV dye for ease of visual observation. A volume of $0.5 \mathrm{~mL}$ of each LHL was added to $100 \mathrm{~mL}$ salt water (34 g/L). Addition of one gram of quartz sand to the floating hydrophobic liquid layer resulted in formation of sand-encapsulated globules, which settled due to increased density. All LHLs (except for a few globules of decane) formed globules covered with fine sand particles that were heavy enough to settle by gravity. The encapsulated globules were stable and retained their shape upon settling. Polarity of hydrophobic liquids as the main factor of aggregation with minerals was found to be insufficient to explain LHL aggregation with sand. Contact angle measurements were made by submerging a large quartz crystal with the LHL drop on its surface into salt water.
\end{abstract}


A positive correlation was observed between the wetting angle of LHL and the LHL volume captured $(r=0.75)$. The dependence of the globule density on globule radius was analyzed in relation to the coverage $(\%)$ of globule surface (LHL-salt water interface) by fine quartz particles.

\section{Introduction}

Formation of so-called 'rafts' with addition of very fine particles to oil slicks was previously described by researchers (Abkarian et al., 2013). Floating crude oil can be effectively captured and its mobility can be controlled with granular materials (e.g., sand) when dry solid particles are added to the light hydrophobic phase liquid (Boglaienko and Tansel, 2015). Addition of granular particles (with higher density) results in formation of particle-covered liquid globules with increased density that settle by gravity. Therefore, crude oil/particle aggregation can be a potential treatment method for reducing the mobility of fresh oil slicks.

Collision of dispersed oil droplets $(<100 \mu \mathrm{m})$ in the marine environment with suspended particulate matter and formation of oil droplets coated with micro-sized solids, both inorganic and organic, but mainly clay, have been studied extensively (Wood et al., 1998; review by Muschenheim and Lee, 2002; Stoffyn-Egli and Lee, 2002; Payne et al., 2003; Bandara et al., 2011; review by Gong et al., 2014).

One of the most common explanations for the process of the creation of oil and suspended material (e.g., clay) aggregates (OSA or OMA) is the polar nature of some components of the crude oil, such as asphaltenes and resins (Bragg and Owens, 1995; Bragg and Yang, 1995; Guyomarch et al., 2002; Khelifa et al., 2002). However, this 
explanation is not sufficient and does not explain the particle-liquid interaction phenomena when larger particles (i.e., sand) are applied on top of a floating hydrophobic liquid for the purpose of its capture and settling.

The purpose of this study is to evaluate the densification of light hydrophobic phases by sand-size particulate matter (or granular particles) for capturing the floating hydrophobic phase, which can settle by gravity after densification. Interaction of light hydrophobic liquids (LHL) with quartz sand was investigated in LHL-salt water systems. Aliphatic and aromatic hydrocarbons that constitute a high percentage of crude oil content (alkanes $80.8 \%$ and aromatics $12.6 \%$, South Louisiana (2001), EPA, 2003) were chosen as model nonpolar compounds. One polar compound (2-chlorotoluene) was added to the experiments to extend the analyses and for comparison. Eight LHLs studied were decane, tetradecane, hexadecane, benzene, toluene, ethylbenzene, m-xylene, and 2-cholorotoluene. Experiments were conducted with fine quartz sand (passing sieve No. 40, openings 0.425 $\mathrm{mm}$ ). Each hydrophobic compound (HC) was dyed with Sudan IV dye for ease of visual observations. The contact angles of HCs with quartz were measured in salt water (34 g/L). A simple model of the density changes of the aggregated globules that accounts for the observations was developed. Dependence of a globule density on globule radius was analyzed in relation to surface coverage (\%) of $\mathrm{HC}$ globules by fine quartz particles.

\section{Materials and Methods}

Hydrophobic liquids and saltwater solution

Eight HCs studied were decane (Fisher Scientific), 99.8\% purity; tetradecane (Fisher Scientific), assay 99.9\%; hexadecane (Fisher Scientific), 99.3\% purity; benzene 
(Fisher Scientific), assay 99.9\%; toluene (Fisher Scientific), assay 99.8\%; ethylbenzene (Fisher Scientific); m-xylene (Fisher Scientific), and 2-chlorotoluene (Aldrich), 99.0\% purity. The HCs were used as provided by the vendor. Synthetic sea salt "Instant Ocean" (Aquarium Systems, Mentor, $\mathrm{OH}$ ) was used at a concentration of $34 \mathrm{~g} / \mathrm{L}$ to prepare the salt water.

\section{Contact angle measurements}

A large quartz crystal (approximately $45 \mathrm{~mm}$ x $25 \mathrm{~mm}$ x $25 \mathrm{~mm}$ ) was washed in methanol and oven dried $\left(100{ }^{\circ} \mathrm{C}\right)$ for an hour. A drop of each hydrophobic liquid (dyed with few crystals of Sudan IV for better visibility) was placed on the quartz surface using a $5-\mathrm{mL}$ graduated pipet, and then the crystal was submerged into salt water $(34 \mathrm{~g} / \mathrm{L})$. Images of the droplets on the crystal surface were captured in both air and after immersing in salt water. Additionally, the wetting characteristics of salt water on quartz were tested as a control.

Contact angles were measured with ImageJ $1.48 \mathrm{v}$ software using the "measure angle" option of the software (National Institutes of Health, USA). Smaller angles $\theta$ (Fig. 4.1) imply better wettability and adhesiveness of the hydrophobic liquid on the solid surface.

The work of adhesion can be estimated by the Young-Dupre equation (Israelachvili, 1985):

$$
W_{123}=\gamma_{23}(1+\cos \theta)
$$

where $\gamma_{23}$ is interfacial surface tension between liquid 2 and salt water 3 ; $W_{123}$ is the reversible work of adhesion of the liquid to the solid surface (Fig. 4.1). For the solid-liquid 
(hydrophobic liquid)-liquid (water) systems, the use of the contact angle measured in the denser phase, or water, $\theta_{w}$ has been suggested (Rao, 2003). Then, the equation (1) becomes:

$$
W_{123}=\gamma_{23}\left(1-\cos \theta_{w}\right)
$$

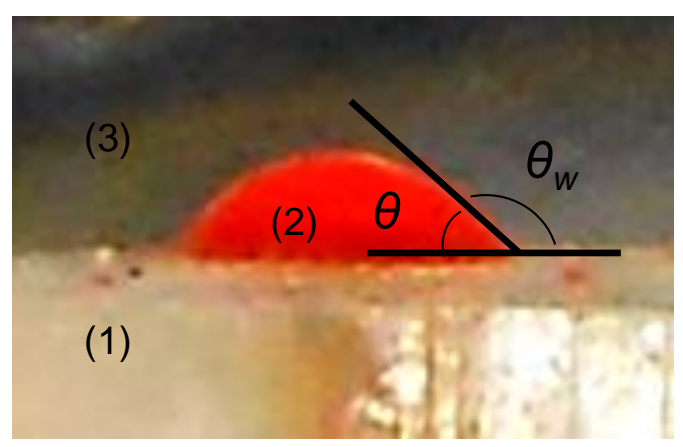

Figure 4.1. Solid-liquid-liquid contact angle measurements: (1) quartz crystal; (2) drop of decane; and (3) salt water medium.

\section{Aggregation experiments with quartz sand}

A set of experiments on the aggregation of the selected hydrophobic compounds (HCs) utilized quartz sand (fine particles that passed sieve No. 40 with opening $0.425 \mathrm{~mm}$ ). Selected hydrophobic compounds represent nonpolar compounds (alkanes: decane, tetradecane, and hexadecane; and monoaromatics, BTEX group: benzene, toluene, ethylbenzene, and m-xylene); and one polar compound, 2-chlorotoluene.

Based on preliminary experiments, the amount of LHL used in the experiments was determined so that there would be a free-floating LHL layer and the globules formed would be easily counted visually. A volume of $0.5 \mathrm{~mL}$ of a selected dyed $\mathrm{HC}$ was added to 100 $\mathrm{mL}$ salt water in a $125 \mathrm{~mL}$ Erlenmeyer flask, using a $10-\mathrm{mL}$ graduated pipet. One gram of 
fine quartz sand was applied on top of the floating slick. Each HC was dyed with few crystals of Sudan IV dye to enhance HC visibility. Additional experiments showed that the dye Sudan IV did not affect the aggregation rates of a $\mathrm{HC}$ with quartz sand (Fig. 4.2a and 4.2b).

Aggregated globules and the number of globules of each size (diameter, mm) were counted manually. The total volume of the aggregated globules was estimated based on the sum of the products of each diameter-class volume by the number of globules in that class. Aggregation experiments were repeated twice for each $\mathrm{HC}$.

Statistical analysis for correlation between the contact angle and aggregated volume of LHL (Pearson coefficient) was computed with help of IBM SPSS 22.
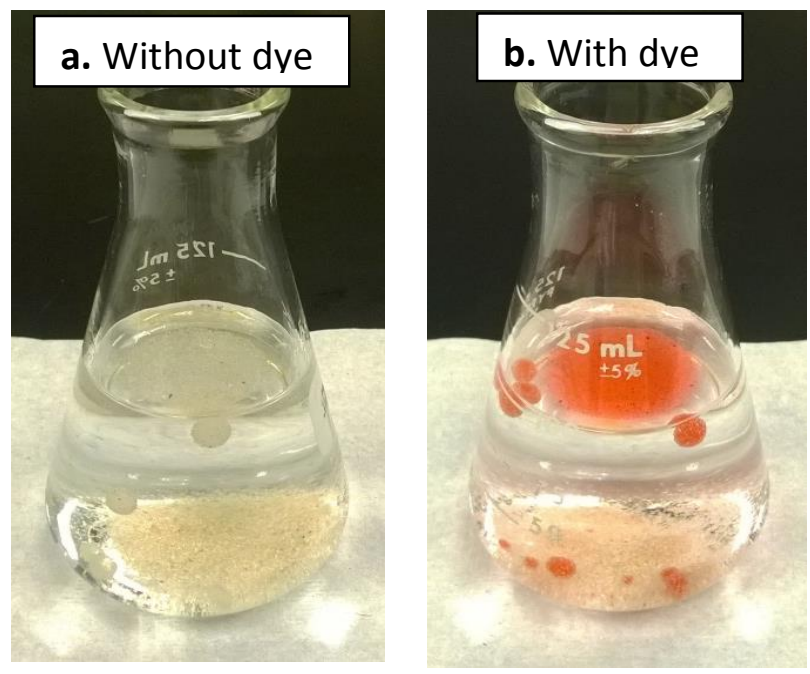


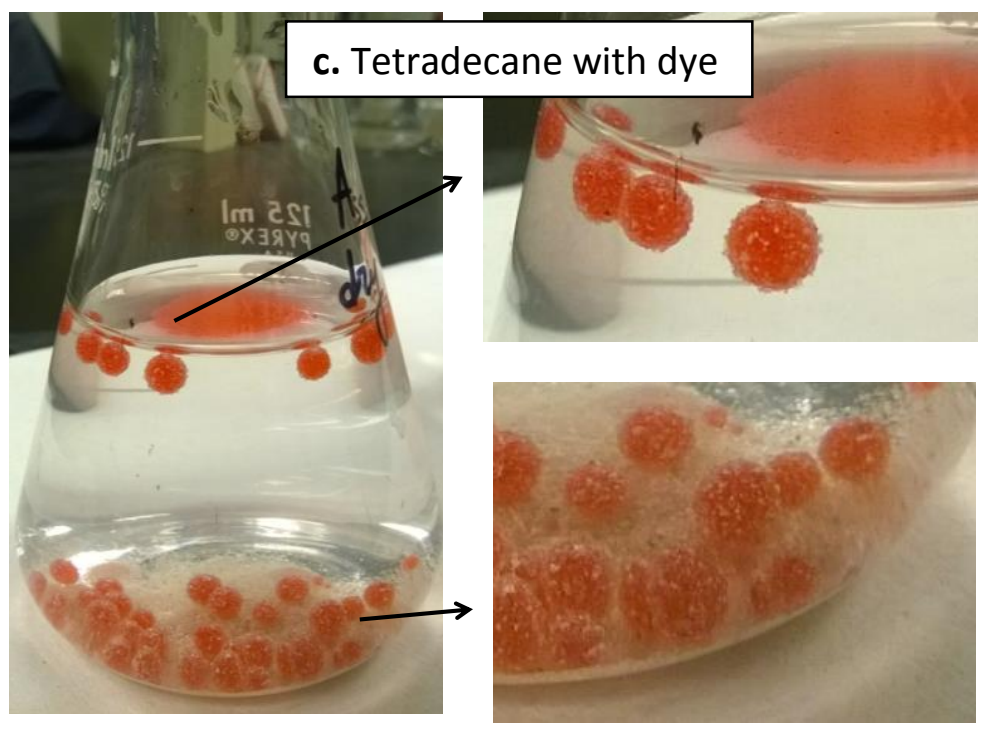

Figure 4.2. LHL-quartz sand interactions in aqueous solutions: a. and b. Aggregation of tetradecane (without dye and with dye) with fine quartz sand; and c. Quartz sandencapsulated tetradecane globules.

\section{Results and Discussion}

\section{Contact angle and encapsulation behavior in salt water}

When the HC drop was placed on the quartz crystal, all the HCs exhibited spreading and complete wetting characteristics when exposed to air (hence effectively a $0^{\circ}$ contact angle); however, when the quartz crystal with the HC drop was immersed in salt water, there was an instantaneous reduction in the wetting characteristics of the HCs as observed from contact angle measurements (Fig. 4.3a). When the salt water drop was placed on the quartz surface and exposed to air, it exhibited a much higher contact angle due to higher surface tension and cohesion forces (by ionic interactions), and hence, lower wetting than LHL. The droplet profile for salt water in air is presented in Fig. 4.3b; and the contact angle $(\theta)$ was measured as $88.2^{\circ}$. 
In air

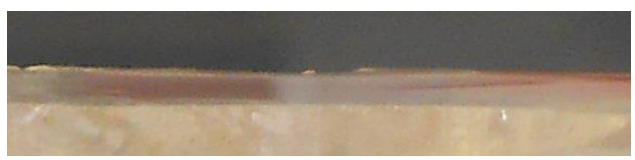

In salt water

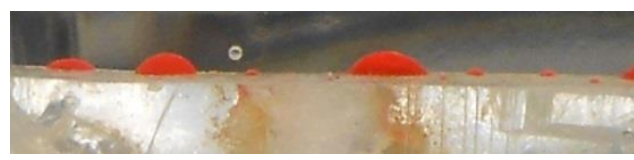

Decane

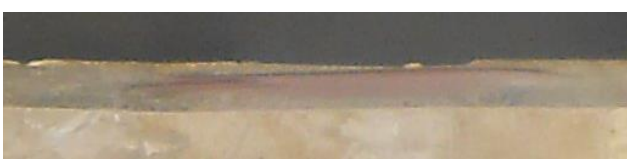

\section{Toluene}

Figure 4.3a. Comparison of wetting characteristics of decane and toluene on quartz surface; exposed to air (total wetting and spreading) and immersed in salt water (hydrophobic behavior).

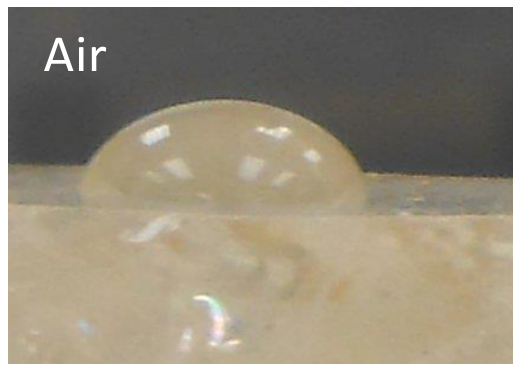

Figure 4.3b. Wetting characteristics of salt water on quartz surface (air as the medium).

Figure 4.3. Wetting characteristics on quartz surface and contact angle measurements.

Table 4.1 presents the contact angle measurements of the HCs on a quartz surface immersed in salt water. We observed increasing solid-liquid-liquid angle $\left(\theta_{w}\right)$ moving from alkanes to aromatics and 2-chlorotoluene (Table 4.1). For all the HCs used, we can consider our system as 'oil-wet', because $\theta_{w}$ were between $115-180^{\circ}$ (Rao, 2003). It should be noted that 2-cholorotoluene is polar while the other HCs studied are nonpolar. The high density and polar nature of 2-cholorotoluene contribute to its relatively higher contact angle $\theta_{w}$ and 
smaller contact angle $\theta$ (and more wetting of the quartz surface) in salt water system in comparison to other HCs.

Table 4.1. Contact angle estimates, density, liquid-water interfacial tension, and aggregated volume of the selected HCs (average of two measurements).

\begin{tabular}{|c|c|c|c|c|c|c|c|}
\hline $\begin{array}{l}\text { Hydrophobic } \\
\text { compound }\end{array}$ & $\begin{array}{c}\text { Contact } \\
\text { angle, } \theta_{w} \\
\text { (degrees) }\end{array}$ & $\operatorname{Cos} \theta_{w}$ & $\begin{array}{l}\operatorname{Cos} \theta \\
\text { [d] }\end{array}$ & $\begin{array}{c}\text { Density } \\
\left(\mathrm{kg} / \mathrm{m}^{3}\right)\end{array}$ & $\begin{array}{c}\text { Liquid- } \\
\text { water } \\
\text { interfacial } \\
\text { tension }^{[\mathrm{b}]} \\
(\mathrm{dyn} / \mathrm{cm})\end{array}$ & $\begin{array}{l}\text { Work of } \\
\text { adhesion } \\
(\mathrm{dyn} / \mathrm{cm})\end{array}$ & $\begin{array}{l}\text { Aggregated } \\
\text { volume } \\
\pm 1 \mathrm{SD}(\%)\end{array}$ \\
\hline Decane & 133.3 & -0.69 & 0.69 & 728 & 52.0 & 87.9 & $31.7 \pm 8.0$ \\
\hline Tetradecane & 142.4 & -0.79 & 0.79 & 758 & 52.2 & 93.4 & $31.8 \pm 16.2$ \\
\hline Hexadecane & 143.2 & -0.80 & 0.80 & 770 & 53.3 & 95.9 & $24.2 \pm 8.7$ \\
\hline Benzene & 158.4 & -0.93 & 0.93 & 873 & 35.0 & 67.6 & $66.9 \pm 8.9$ \\
\hline Toluene & 151.0 & -0.88 & 0.88 & 865 & 36.1 & 67.5 & $49.6 \pm 13.6$ \\
\hline Ethylbenzene & 149.5 & -0.86 & 0.86 & 865 & 38.4 & 71.4 & $67.1 \pm 9.3$ \\
\hline m-Xylene & 144.8 & -0.82 & 0.82 & 861 & 37.9 & 69.0 & $46.1 \pm 18.9$ \\
\hline $\begin{array}{l}\text { 2-Chloro- } \\
\text { toluene }\end{array}$ & 164.8 & -0.97 & 0.97 & 1077 & $37.1^{[\mathrm{c}]}$ & 73.1 & $91.3 \pm 5.5$ \\
\hline
\end{tabular}

Encapsulation and densification of LHLs with quartz sand

Quartz sand addition was most effective in densification of 2-chlorotoluene. In the 2-chlorotoluene-salt water system, almost all the floating phase settled with distinctly larger size globules. The density of 2-chlorotoluene is very close to the density of salt water, hence, even a small coverage with sand particles makes globules sink. Some of the 2-chlorotoluene globules did not have sand particles on the upper half of their surfaces but settled (by gravity) due to the high density of 2-chlorotoluene. Moreover, 2-chlorotoluene 
was the only hydrophobic compound (among the 8 studied) that formed globules of relatively large size (up to $8 \mathrm{~mm}$ in diameter).

Decane also had distinct behavior because of its low density and low wetting characteristics indicated by the smallest contact angle $\left(\theta_{w}=133.3^{\circ}\right.$, Table 4.1$)$ with quartz in salt water. As a result of the bouncing and impact of the globule upon reaching the bottom (as it settled by gravity), some sand particles were detached from the globule surface; reducing the globule density. Hence, a few of the globules (1-2 globules) formed in the decane-salt water system floated up in the salt water (Fig. 4.4a and 4.4b).

The total volume of globules formed and settled in relation to the initial amount of floating hydrophobic layer present in salt water for each HC is given in Table 4.1 (as the average of two replicate trials $\pm 1 \mathrm{SD})$. A positive correlation (Pearson $r(16)=0.75, p=$ 0.001) was observed between the aggregated (and gravity separated) volume of the hydrophobic liquids with the quartz-hydrophobic liquid contact angle (measured in salt water), as presented in Fig. 4.5. Relationship between the estimates of work of adhesion and the aggregated volume did not reveal clear direction. 


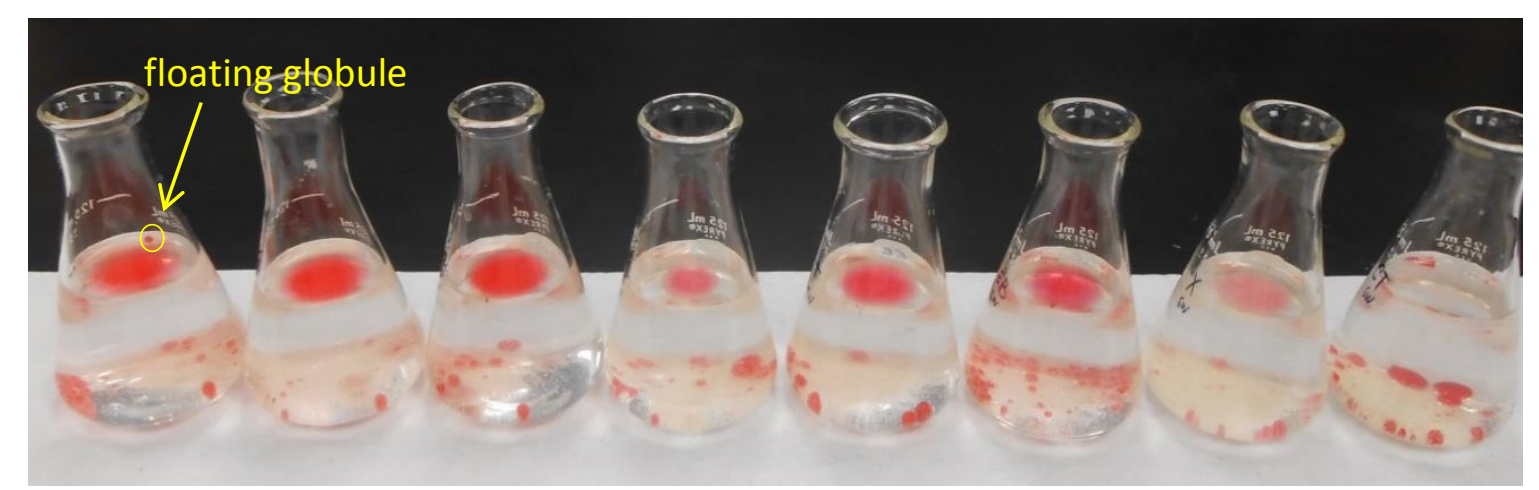

\begin{tabular}{|llllllll|}
\hline Decane & Tetradecane & Hexadecane & Benzene & Toluene & Ethylbenzene & m-Xylene & $\begin{array}{l}\text { 2-Choloro- } \\
\text { toluene }\end{array}$ \\
$\mathrm{C}_{10} \mathrm{H}_{22}$ & $\mathrm{C}_{14} \mathrm{H}_{30}$ & $\mathrm{C}_{16} \mathrm{H}_{35}$ & $\mathrm{C}_{6} \mathrm{H}_{6}$ & $\mathrm{C}_{7} \mathrm{H}_{8}$ & $\mathrm{C}_{8} \mathrm{H}_{10}$ & $\mathrm{C}_{8} \mathrm{H}_{10}$ & $\mathrm{C}_{7} \mathrm{H}_{7} \mathrm{Cl}$ \\
\hline
\end{tabular}

Figure 4.4a. Formation of encapsulated light hydrophobic globules after addition of quartz sand in LHL-salt water systems.
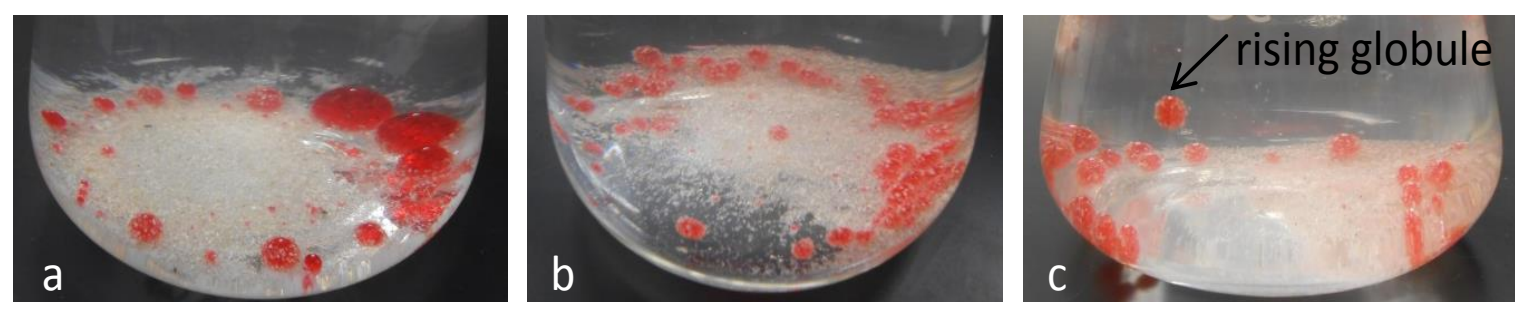

Figure 4.4b. Comparison of the encapsulated globules formed in LHL-salt water systems after addition of quartz sand. LHLs with higher densities formed larger globules with less sand coverage: a. 2-cholorotoluene $\left(\mathrm{d}=1077 \mathrm{~kg} / \mathrm{m}^{3}\right)$; b. Ethylbenzene $\left(\mathrm{d}=865 \mathrm{~kg} / \mathrm{m}^{3}\right)$; and c. Decane $\left(\mathrm{d}=728 \mathrm{~kg} / \mathrm{m}^{3}\right)$.

Figure 4.4. Encapsulation of hydrophobic liquids with fine quartz sand. 


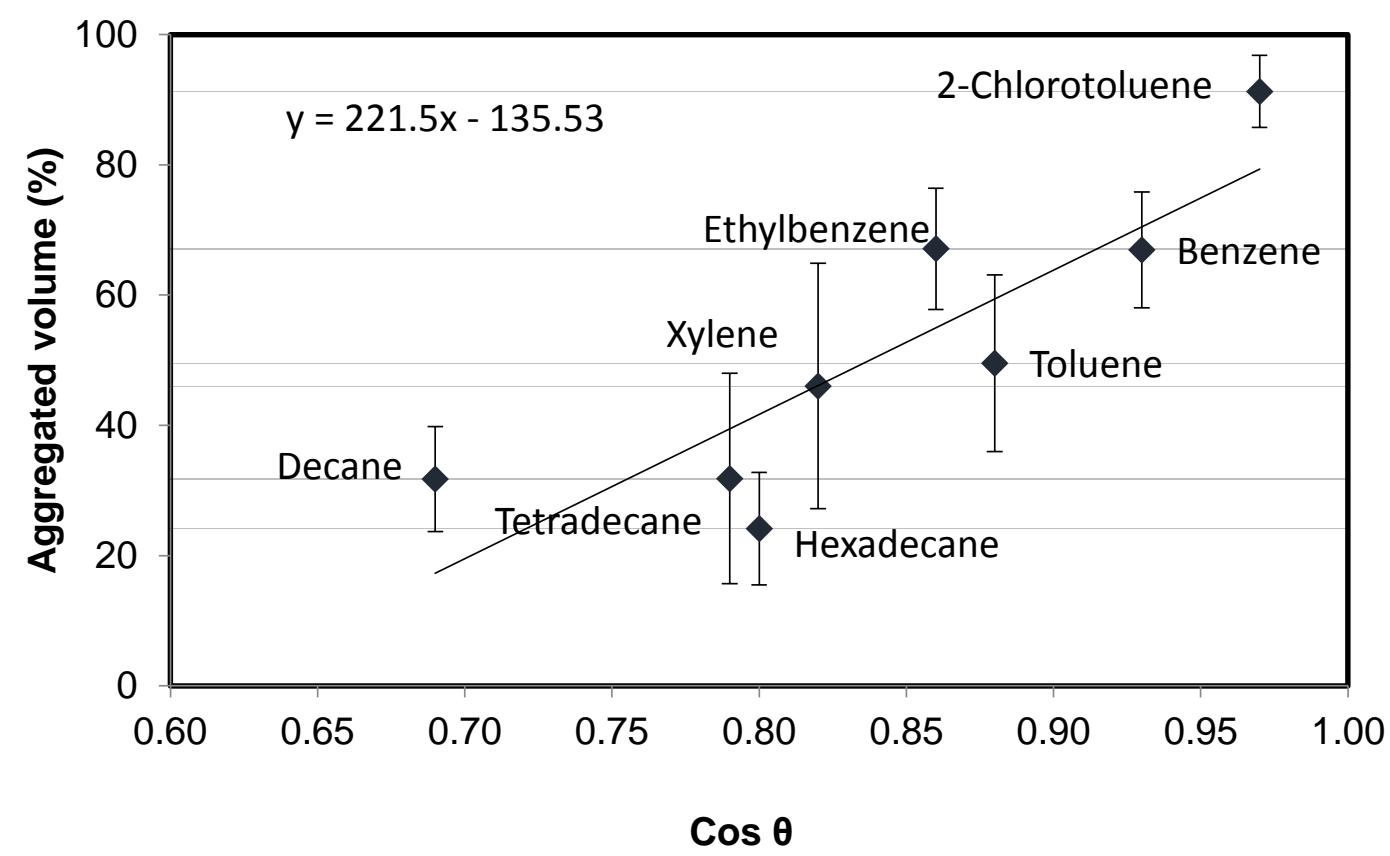

Figure 4.5. Correlation of the aggregated and gravity-separated volume of the hydrophobic liquids with the quartz-hydrophobic liquid contact angle (measured in salt water); $r^{2}=0.56$.

\section{Modeling of globule formation and behavior}

The density of the aggregated globule controls its settling behavior. If the density is larger than the density of the water phase (i.e., salt water, $1024 \mathrm{~kg} / \mathrm{m}^{3}$ at $34 \%$ salinity and $20^{\circ} \mathrm{C}$ ), it settles, and if it is smaller, the globule floats. The density of a globule can be estimated as follows:

$$
\rho_{g}=\frac{M h c+M p}{V g},
$$

where $M h c$ is mass of the $\mathrm{HC}$ that is being aggregated, $\mathrm{kg} ; M p$ is mass of the quartz particles that adhere to the globule's surface; $\mathrm{kg} ; \mathrm{Vg}$ is volume of the globule, $\mathrm{m}^{3}$.

Assuming spherical globules, the mass of $\mathrm{HC}$ in the globule is:

$$
M h c=\rho_{h c}\left(\frac{4}{3}\right) \pi r_{g}^{3}
$$


where $\rho_{h c}$ is density of the $\mathrm{HC} ; \mathrm{kg} / \mathrm{m}^{3} ; r_{g}$ is radius of the globule, $\mathrm{m}$.

Mass of particles:

$$
M p=n M
$$

where $n$ is number of the quartz particles on the globule's surface; $M$ is mass of one quartz particle, $\mathrm{kg}$.

Assuming a particle of quartz is a sphere with radius $r_{p}$, then:

$$
M=\rho_{p}\left(\frac{4}{3}\right) \pi r_{p}^{3}
$$

where $\rho_{p}$ is the density of quartz, $2650 \mathrm{~kg} / \mathrm{m}^{3} ; r_{p}$ : radius of quartz particles, $\mathrm{m}$.

The number of particles attached on the HC globule surface can be estimated by dividing the surface area of the globule by the area occupied by one particle (assuming the occupied area is circular):

$$
n=\frac{4 \pi r_{g}^{2}}{\pi r_{p}^{2}} K_{c o v}
$$

where $K_{c o v}$ is percentage of a globule surface, covered with sand particles.

After combining equations (4)-(7) with equation (3), the density of $\mathrm{HC}$ globule can be estimated by the following equation:

$$
\rho_{g}=\frac{\rho_{h c}\left(\frac{4}{3}\right) \pi r_{g}^{3}+\frac{4 \pi r_{g}^{2}}{\pi r_{p}^{2}} K_{c o v} M}{\left(\frac{4}{3}\right) \pi r_{g}^{3}}
$$

After simplification:

$$
\rho_{g}=\frac{\rho_{h c} r_{g}+4 K_{\operatorname{cov}} \rho_{p} r_{p}}{r_{g}}
$$

Figure 4.6 presents the estimated densities of the globules in relation to globule diameter and percentage of surface coverage by quartz sand particles for the eight HCs used in this study. The density-coverage model supported the experimental observations. 
For example, for the 2-chlorotoluene with density very close to the density of salt water; a small coverage with sand particles will make globules become unstable and sink. It should be noted that high cohesive behavior of the hydrophobic compounds allows formation of the larger globules especially when their density is closer to that of salt water.

However, formation of a gas bubble on top of aggregated globules of HCs was observed a couple of hours later. Equation (3) then becomes:

$$
\rho_{g}=\frac{M h c+M p+M g a s}{V g+V g a s}
$$

That is

$$
\rho_{g}=\frac{\rho_{h c}\left(\frac{4}{3}\right) \pi r_{g}^{3}+\frac{4 \pi r_{g}^{2}}{\pi r_{p}^{2}} K_{c o v} M+\rho_{\text {gas }}\left(\frac{4}{3}\right) \pi r_{\text {gas }}^{3}}{\left(\frac{4}{3}\right) \pi r_{g}^{3}+\left(\frac{4}{3}\right) \pi r_{g a s}^{3}}
$$

where $\rho_{\text {gas }}$ is density of the gas bubble, $\mathrm{kg} / \mathrm{m}^{3} ; r_{\text {gas }}$ is radius of the gas bubble, $\mathrm{m}$.

After simplification:

$$
\rho_{g}=\frac{\rho_{h c} r_{g}^{3}+4 K_{\operatorname{cov}} \rho_{p} r_{p} r_{g}^{2}+\rho_{g a s} r_{g a s}^{3}}{r_{g}^{3}+r_{g a s}^{3}}
$$

Figure 4.6 presents results for the freshly formed globules (right after the sand application), when gas phase has not been developed yet.

Gas phase may be caused by air entrapment and volatilization of the liquids. The amount of air entrapped within the globule is highly correlated with the way sand is applied. When sand application is gradual, air entrapment is relatively small. The gas bubble emerged over time (volatilization inside the encapsulation) and eventually escaped from the globule. Therefore, in most cases, the bubble did not alter the behavior of the globules, as care was taken to minimize air entrapment. 
This model can be applied to any HC-particle pairs of interest. The globule size may be controlled by the interaction of particle characteristics (size, density), wettability, and particle application method, as it will affect the number of granular particles covering a globule (coverage percent $K_{\text {cov }}$ ) and, thus, densification rates of a LHL. Additional experiments to evaluate, for example, the impact of particle size, composition, and surface properties and surface treatments, as well as the number (mass) of particles and their method of application to the slick, could be devised to optimize capture and settling.
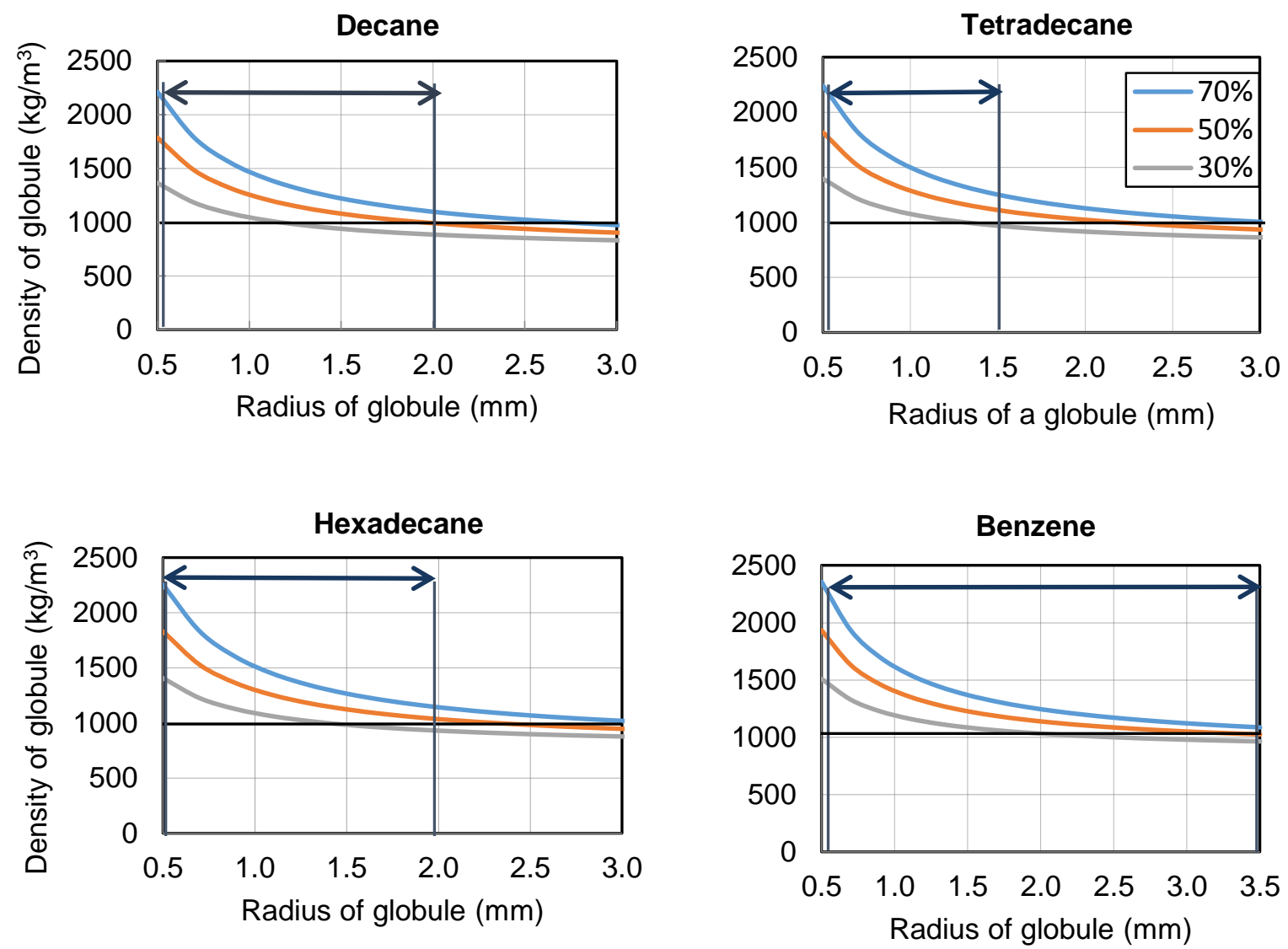

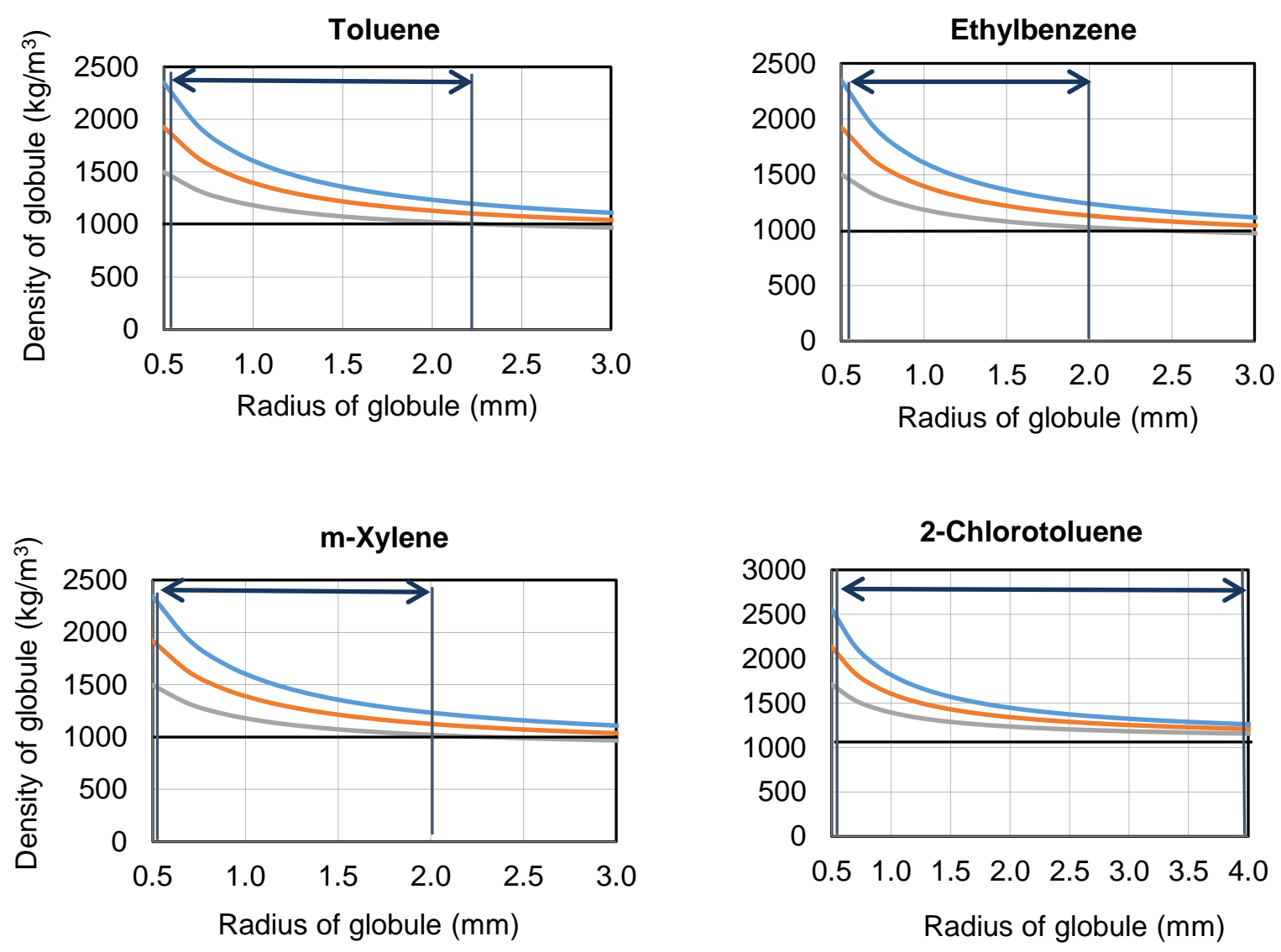

Figure 4.6. Variation of globule density with its radius $(\mathrm{mm})$ and surface coverage $(\%)$ by fine quartz particles. Blue, red, and gray lines: 70,50 , and $30 \%$ of sand coverage of a globule respectively. Globules with densities greater than the water phase (shown as a horizontal black line) settle. Vertical lines show the range of observed globule size formed during the experiments. Modelling was made for the globules of fresh formation (gas phase on top of a globule was not accounted for).

\section{Conclusions}

Surface application of quartz sand effectively captured the floating hydrophobic phase by forming globules of hydrophobic liquids that settled by gravity. The quartz sand particles were observed to encapsulate the globules of hydrophobic liquids, with partial coverage on the hydrophobic phase-salt water interface. All hydrophobic liquids formed stable globules encapsulated with fine quartz sand particles that settled by gravity. A few 
globules formed in the decane-salt water system floated back due to loss of granular particles (due to the globules bouncing off the bottom) and the low density of decane.

Even though the polar compound (2-chlorotoluene) had the highest aggregated volume $(91.3 \pm 5.5 \%)$ and better wettability with quartz, experimental results reveal the ability of nonpolar constituents of crude oil (alkanes and aromatics) to aggregate with sand particles. The abovementioned assumption (see Introduction) that crude oil aggregates with mineral particles because of the presence of polar compounds in its content, does not satisfy our experimental results.

A positive correlation was observed between the wettability of quartz by the hydrophobic liquids and the amount of hydrophobic liquid captured (as encapsulated globules) by application of quartz sand (Pearson $r=0.75, p=0.001$ ). Use of granular materials for densification of floating hydrophobic liquids can be an effective technology for controlling mobility, hence, reducing the impact radius of oil slicks at sea.

\section{Acknowledgments}

Partial funding for this research has been provided by Gulf of Mexico Research Initiative (GoMRI) through funding to Consortium for the Molecular Engineering of Dispersant Systems (CMEDS).

\section{References}

Abkarian, M., Protiere, S., Aristoff, J.M., and Stone, H.A. 2013. Gravity-induced encapsulation of liquids by destabilization of granular rafts. Nature Communications, 4 (1895). 
Bandara, U.C., Yapa, P.D., and Xie, H. 2011. Fate and transport of oil in sediment laden marine waters. Journal of Hydro-environmental Research, 5 (3): 145-156.

Boglaienko, D., and Tansel, B. 2015. Instantaneous stabilization of floating oils by surface application of natural granular materials (beach sand and limestone). Marine Pollution Bulletin, 91 (1): 107-112.

Bragg, J.R., and Owens, E.H. 1995. Shoreline cleansing by interactions between oil and fine mineral particles. In Proceedings of the International Oil Spill Conference, Feb. 22-Mar 2, Long Beach, CA, American Petroleum Institute, Washington, DC, Publication No. 4620, pp. 219-227.

Bragg, J.R, and Yang, S.H. 1995. Clay-oil flocculation and its effects on the rate of natural cleansing in Prince William Sound following the Exxon Valdez oil spill. In: Wells, P.G., Butler, J.N., Hughes, J.S. (Eds.), Exxon Valdez Oil Spill - Fate and Effects in Alaskan Waters. American Society for Testing and Materials, Philadelphia, PA. ASTM STP 1219, pp. 178-214.

Demond, A.H., and Lindner, A.S. 1993. Estimation of interfacial tension between organic liquids and water. Environmental Science \& Technology, 27: 2318-2331.

EPA/600/R-03/072. USEPA. 2003. Characteristics of spilled oils, fuels, and petroleum products: 1 . Composition and properties of selected oils.

Gong, Y., Zhao, X., Cai, Z., O'Reilly, S.E., Hao, X., and Zhao, D. 2014. A review of oil, dispersed oil and sediment interactions in the aquatic environment: influence on the fate, transport and remediation of oil spills. Marine Pollution Bulletin, 79 (1-2): 1633 .

Guyomarch, J., Le Floch, S., and Merlin, F.-X. 2002. Effect of suspended mineral load, water salinity and oil type on the size of oil-mineral aggregates in the presence of chemical dispersant. Spill Science \& Technology Bulletin, 8 (1): 95-100.

Israelachvili, J.N. 1985. Intermolecular and surface forces: with applications to colloidal and biological systems. London: Academic Press.

Jasper, J.J., and Seitz, H.R. 1958. The temperature-interfacial tension studies of some halogenated toluenes against water. The Journal of Physical Chemistry, 62 (10): 1331-1333.

Khelifa, A., Stoffyn-Egli, P., Hil, P.S., and Lee, K. 2002. Characteristics of oil droplets stabilized by mineral particles: effects of oil type and temperature. Spill Science \& Technology Bulletin, 8 (1): 19-30. 
Muschenheim, D.K., and Lee, K. 2002. Removal of oil from the sea surface through particulate interactions: review and prospectus. Spill Science \& Technology Bulletin, 8 (1): 9-18.

Payne, R.J., Clayton, Jr. J.R., and Kirstein, B.E. 2003. Oil/Suspended particulate material interactions and sedimentation. Spill Science \& Technology Bulletin, 8 (2): 201221.

Rao, D.N. 2003. The concept, characterization, concerns, and consequences of contact angles in solid-liquid-liquid systems. In: Mittal, K. L. (Ed.), Contact Angle, Wettability and Adhesion, Vol. 3. Leiden, NLD: Brill Academic Publishers.

Stoffyn-Egli, P., and Lee, K. 2002. Formation and characterization of oil-mineral aggregates. Spill Science \& Technology Bulletin, 8 (1): 31-44.

Wood, P.A., Lunel, T., Daniel, F., Swannell, R., Lee, K., and Stoffyn-Egli, P. (1998). Influence of oil and mineral characteristics on oil-mineral interaction. Proceedings of the twenty-first Arctic and marine oil spill program (AMOP) technical seminar. Environment Canada, Ottawa, Ont., pp. 51-77.

Yaws, C.L. 1999. Chemical properties handbook: physical, thermodynamic, environmental, transport, safety, and health related properties for organic and inorganic chemicals. McGrown-Hill. 


\title{
CHAPTER V
}

\section{ENCAPSULATION OF LIGHT HYDROPHOBIC LIQUIDS WITH FINE QUARTZ SAND: PROPERTY BASED CHARACTERIZATION AND STABILITY IN AQUEOUS MEDIA WITH DIFFERENT SALINITIES}

Boglaienko, D., and Tansel, B. 2016. Chemical Engineering Science, 145: 90-96.

DOI:10.1016/j.ces.2016.02.010

\begin{abstract}
A novel method for capturing oil slicks in aquatic environments with granular solids was investigated. Experiments were conducted with pure hydrophobic liquids (HL) (decane, tetradecane, hexadecane, benzene, toluene, ethylbenzene, and m-xylene) representing the main groups in crude oil (alkanes and aromatics), and fine quartz sand (passing sieve No. 40 with $0.425 \mathrm{~mm}$ openings). The amounts of HL captured in the HL/sand globules were correlated with the properties the HLs. Positive correlations between the aggregated HL volume with density and surface tension were observed. Aggregation number $\left(A_{N}\right)$ is introduced to estimate the amount of HL aggregated with fine quartz sand (using $0.5 \mathrm{~mL} \mathrm{HL}$ and $1.0 \mathrm{~g}$ sand; floating HL layer thickness to particle diameter ratio > 4:1). Change in the aggregated HL volume over time was examined and dissolution rate constants were obtained. Benzene and toluene completely dissolved from the aggregated globules within 3-4 days after the aggregation, while the other HLs exhibited relatively small change in the globule size over time. Salinity of water did not affect the aggregation rates of HLs but slightly decreased the dissolution rate constants for some HLs.
\end{abstract}




\section{Introduction}

Floating oil slicks can be effectively captured and submerged with dry fine granular materials, such as quartz sand or limestone (Abkarian, 2013; Boglaienko and Tansel, 2015). This method is unique in its simplicity and availability of natural granular materials in coastal areas; therefore, it can be an effective method for use in marine environments to control spreading of floating oil slicks. Once the granular material comes into contact with the floating slick of a hydrophobic liquid (e.g., crude oil); it accumulates on the floating slick, gradually increasing its density. As the oil slick carrying the granular particles gets heavier by the addition of the dense granular particles; liquid/solid globular aggregates sink rapidly when the coverage of globules is sufficient to create denser than water aggregates. The shape and characteristics of the HL/solid particle globules depend on the particle size of the granular solid and characteristics of the hydrophobic liquid (HL). The gravityinduced capture and transformation of an oil slick from the light floating phase to heavy sinking phase has certain advantages from an environmental impact perspective. Transforming floating oil from water surface to subsurface allows better control of the fate and transport of oil slicks in the environment by keeping the impact footprint smaller, and allow use of controlled addition of nutrients for faster decomposition of the oil in the subsurface (i.e., underwater bioremediation).

In the studies that examined aggregation of suspended mineral particles with dispersed in water oil droplets (Bragg and Owens, 1995; Bragg and Yang, 1995; Wood et al., 1998; Muschenheim and Lee, 2002; Stoffyn-Egli and Lee, 2002; Guyomarch et al., 2002; Khelifa et al., 2002; Payne et al., 2003; Bandara et al., 2011; Gong et al., 2014), amount of polar compounds in oil was referred to as a crucial factor in creation of 
hydrophobic bonding between minerals and the surface of oil droplets (Stoffyn-Egli and Lee, 2002). Oils with higher content of polar compounds (resins and asphaltenes) were found to aggregate better with fine particulate matter but the impact of higher viscosity of these oils on the better aggregation rates was not considered (Sørensen, 2014). The main goal of this research was to study the aggregation rates of HLs in relation to different properties (density, viscosity, surface and interfacial tensions). As the representatives of alkane group we chose decane, tetradecane, and hexadecane; and of aromatic group benzene, toluene, ethylbenzene, and m-xylene (BTEX). For the purpose to compare the mentioned nonpolar compounds to polar ones, we utilized only 2-chlorotoluene (electronegativity of $\mathrm{Cl}$ is $3.0, \mathrm{C}$ is 2.5 , and $\mathrm{H}$ is 2.2 (Schwarzenbach, 1993), as the most polar liquids dissolve in water very well and are not suitable for the experiments. Experiments were carried out in salt water and deionized water, as it was stated in literature that saline water is the prerequisite for suspended oil and clay interactions (Lee, 2002). The statement that cations of salt water act as electrical bridges helping aggregation of suspended clay particles and dispersed oil (Brag and Yang, 1995) may not be true for our case, when the fine particles hit the floating slick before reaching water.

However, as our preliminary results showed, for the system of the direct application of granular particles on surface of the floating oil, even nonpolar compounds as alkanes (tested $\mathrm{C} 5, \mathrm{C} 10, \mathrm{C} 14$, and C16) create globular aggregates. We relate this to the Van der Waals or dispersion forces between HLs and quartz sand particles that cover the surface of the aggregated globules (Israelochvilli, 1985; Hunter, 2001).

Considering toxicity of aromatic compounds, which are the most toxic group in crude oil content (Atlantic, 1988), we looked at the dissolution rates of the selected HL 
from the aggregated globules with time, both in salt and deionized water. We expected the aggregates to be more stable in salt water due to "salting out" effect, which explains decreased solubility of nonpolar organics in water with ions of inorganic salts (Schwarzenbach, 1993).

Presented experiments were done for the quartz sand only, however, various materials may have different aggregation rates due to surface chemistry (Sterling, 2004).

In our study, we investigated the effect of salinity on the aggregation rates $(34 \%$ o synthetic saltwater versus deionized water); the correlations between the granular aggregation rates and the properties of the selected HLs; and the stability of the aggregates over time. The HLs we evaluated represent the main constituents of crude oil, alkanes and aromatics.

\section{Materials and Methods}

Decane, tetradecane, hexadecane, benzene, toluene, ethylbenzene, and m-xylene were obtained from Fisher Scientific (purity > 99.0\%), and 2-chlorotoluene from Aldrich (99.0\% pure). The hydrocarbons were dyed with a few crystals of Sudan IV dye (additional trials showed that the dye did not affect the aggregation rates). Synthetic sea salt 'Instant Ocean' (Aquarium Systems, Mentor, OH) was used to prepare 34\%o salt water. Fine quartz sand (passed sieve No. 40 with opening $0.425 \mathrm{~mm}$ ) was used for aggregation and applied at the amount of $1.00 \pm 0.01 \mathrm{~g}$ to the surface of the floating hydrophobic liquid $(0.50 \pm$ $0.06 \mathrm{~mL}$ ). The amount of sand was chosen according to our previous experimental results (Boglaienko and Tansel, 2015), where the range of granular material to oil ratios was studied utilizing an orbital shaker to simulate sea turbulence (150 rpm). Aggregation 
experiments were performed in a $125 \mathrm{~mL}$ Erlenmeyer flask filled with $100 \mathrm{~mL}$ of salt water or with $100 \mathrm{~mL}$ of deionized water.
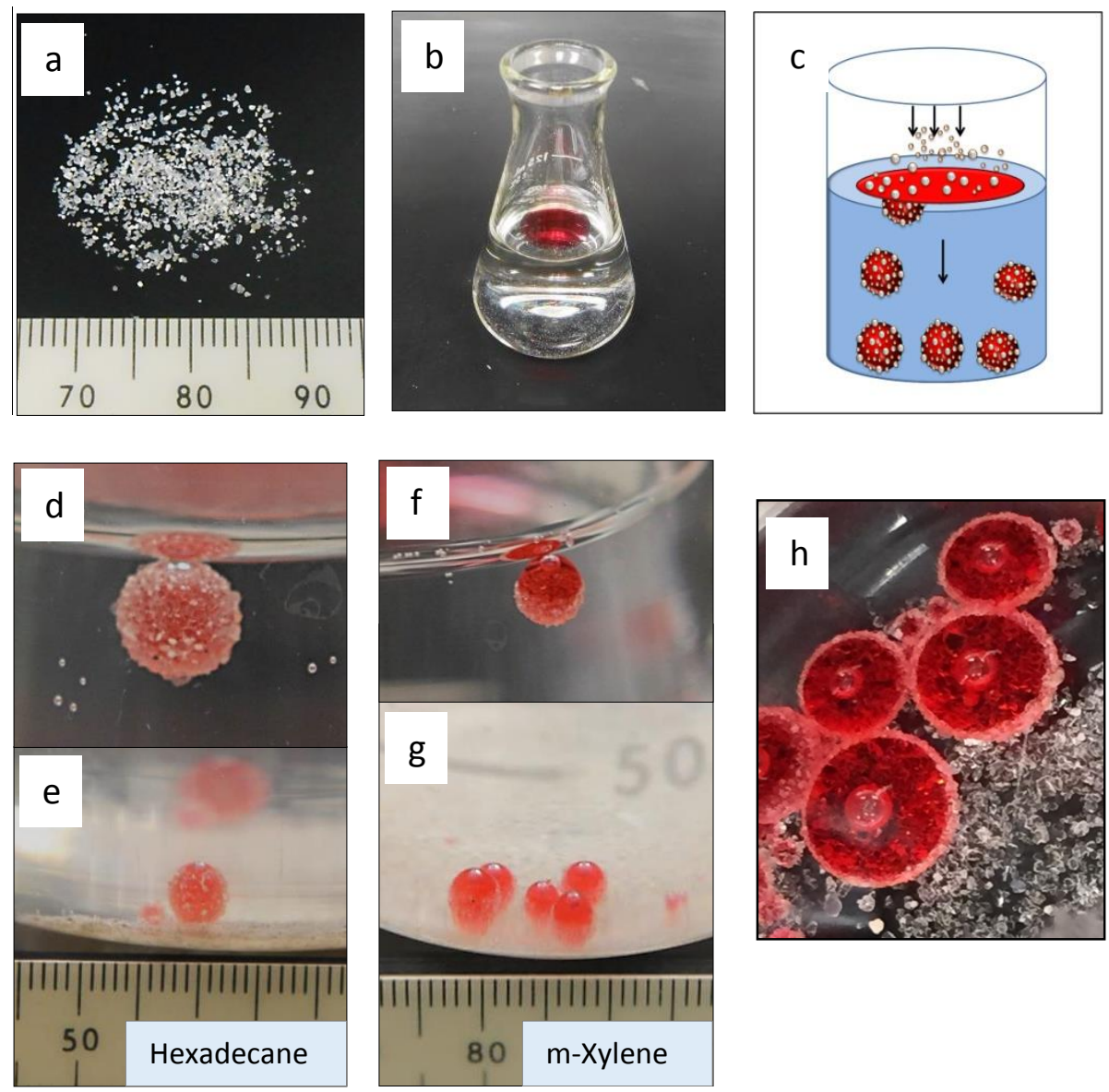

Figure 5.1. Materials and aggregated globules: a. Fine quartz sand; b. Erlenmeyer flask with salt water $(100 \mathrm{~mL})$ and floating layer of a dyed $\mathrm{HL}(0.5 \mathrm{~mL})$; c. Conceptual schematic of adding sand to HL slick; d. and e. Floating and submerged globules of hexadecane aggregated with fine quartz sand; f. and g. Floating and submerged globules of $m$-xylene aggregated with fine quartz sand; and $\mathbf{h}$. Close up view (top) of the submerged globules of $\mathrm{m}$-xylene aggregated with fine quartz sand.

Sand particles, reaching the floating layer, initiated instantaneous aggregation of the floating hydrophobic liquid (HL) to form globules ranging from 1 to $8 \mathrm{~mm}$ in diameter (size range varied depending on the HL). The total volume of the aggregated HL was 
determined from the number of globules formed and their diameters. The globules formed were spherical or near spherical shape. The size and number of globules formed were monitored every three days during a 10 -day period $\left(1^{\text {st }}, 4^{\text {th }}, 7^{\text {th }}\right.$, and $10^{\text {th }}$ day $)$. The globules formed with the most volatile compounds, benzene and toluene, were monitored every day during the first four days due to noticeable change in the globule size. The experiments were performed four times, twice with salt water and twice with deionized water. The estimated volume of the aggregated HL on the first day (fresh) were utilized for the property analysis, and the data on the dynamics of the volume of aggregated HL within 10 days were used for the time dependent analysis (i.e., rate of change in captured volume).

In analogy to adsorption coefficient, $K_{d}\left[\mathrm{~L}^{3} / \mathrm{M}\right]$, that describes the adsorption capacity of a substance to solid material, we defined the aggregation coefficient, $K_{a g}$ $\left[\mathrm{L}^{3} / \mathrm{M}\right]$, which represents the volume of the aggregated HL per unit mass of granular solid material:

$$
K_{a g}=\frac{V_{a g}}{M_{\text {solids }}}
$$

where, $V_{a g}$ is volume of HL liquid (mL), $M_{\text {solids }}$ is the mass of granular particles $(\mathrm{g})$, and $K_{a g}$ is the aggregation coefficient $(\mathrm{mL} / \mathrm{g})$.

\section{Results}

Table 5.1 summarizes the total volume of HL aggregates and the percent from the initial volume in salt water and deionized water (first day). Experiments were conducted under conditions when the thickness of the floating HL layer was more than 4 times larger than the particle size (estimated knowing the geometry and volume of the HL floating layer 
and the particle size passing through the sieve No. 40). The aggregation rates were correlated with the physical properties of the HLs (Table 5.2, Fig. 5.2).

Table 5.1. Aggregation coefficient $K_{a g}(\leq 0.5 \mathrm{~mL})$ and percent of the aggregated volume from the initial $(0.5 \mathrm{~mL})$ for each HL. Data are averages of two sets; standard deviation is given in parenthesis. Layer thickness to particle diameter ratio is $>4: 1$.

\begin{tabular}{llllll}
\hline \multirow{2}{*}{$\begin{array}{l}\text { Hydrophobic } \\
\text { liquid }\end{array}$} & \multicolumn{3}{c}{ Salt water } & & \multicolumn{2}{c}{ Deionized water } \\
\cline { 2 - 3 } \cline { 5 - 6 } & $K_{a g}, \mathrm{~mL} / \mathrm{g}$ & $\begin{array}{l}\text { Volume, \% of } \\
\text { initial } 0.5 \mathrm{~mL}\end{array}$ & & $K_{a g}, \mathrm{~mL} / \mathrm{g}$ & \multicolumn{1}{l}{$\begin{array}{l}\text { Volume, \% of initial } \\
0.5 \mathrm{~mL}\end{array}$} \\
\hline Decane & 0.17 & $34.9(4.9)$ & & 0.15 & $29.7(9.1)$ \\
Tetradecane & 0.08 & $16.8(11.9)$ & & 0.12 & $23.5(5.5)$ \\
Hexadecane & 0.12 & $23.1(3.0)$ & & 0.13 & $25.9(13.9)$ \\
Benzene & 0.24 & $48.1(13.8)$ & & 0.20 & $40.3(8.3)$ \\
Toluene & 0.15 & $30.8(2.6)$ & & 0.27 & $53.5(0.9)$ \\
Ethylbenzene & 0.26 & $52.1(5.9)$ & & 0.24 & $48.4(10.1)$ \\
m-Xylene & 0.14 & $28.0(0.7)$ & & 0.29 & $57.6(6.5)$ \\
2-Chlorotoluene & 0.47 & $94.4(4.2)$ & & 0.47 & $94.9(1.7)$ \\
\hline
\end{tabular}

Table 5.2. Selected properties of the hydrophobic liquids.

\begin{tabular}{|c|c|c|c|c|c|c|c|}
\hline $\begin{array}{l}\text { Hydrophobic } \\
\text { liquid }\end{array}$ & Formula & $\begin{array}{l}\text { Molecular } \\
\text { weight }^{[\mathrm{a}]} \\
(\mathrm{g} / \mathrm{mol})\end{array}$ & $\begin{array}{c}\text { Density }^{[\mathrm{a}]} \\
(\mathrm{g} / \mathrm{mL})\end{array}$ & $\begin{array}{l}\text { Viscosity } \\
(\mathrm{mPa}]\end{array}$ & $\begin{array}{l}\text { Surface } \\
\text { tension }{ }^{[\mathrm{a}]} \\
\left(\mathrm{mN} / \mathrm{m}^{2}\right)\end{array}$ & $\begin{array}{l}\text { Interfacial } \\
\text { tension } \\
\text { with } \\
\text { water } \\
(\mathrm{m} \text { [b],[c] } \\
\left(\mathrm{m}^{2}\right)\end{array}$ & $\begin{array}{l}\text { Solubility in } \\
\text { salt water / } \\
\text { Solubility in } \\
\text { water }{ }^{[\mathrm{a}],\left[{ }^{*}\right]}\end{array}$ \\
\hline Decane & $\mathrm{C}_{10} \mathrm{H}_{22}$ & 142.285 & 0.728 & 0.863 & 23.41 & 52.0 & $0.0385 / 0.0520$ \\
\hline Tetradecane & $\mathrm{C}_{14} \mathrm{H}_{30}$ & 198.392 & 0.758 & 2.110 & 26.15 & 52.2 & $0.0017 / 0.0022$ \\
\hline Hexadecane & $\mathrm{C}_{16} \mathrm{H}_{34}$ & 226.446 & 0.770 & 3.063 & 27.09 & 53.3 & $0.0004 / 0.0009$ \\
\hline Benzene & $\mathrm{C}_{6} \mathrm{H}_{6}$ & 78.114 & 0.873 & 0.606 & 28.21 & 35.0 & $1354.0 / 1755.0$ \\
\hline Toluene & $\mathrm{C}_{7} \mathrm{H}_{8}$ & 92.141 & 0.865 & 0.548 & 27.93 & 36.1 & $402.0 / 542.4$ \\
\hline Ethylbenzene & $\mathrm{C}_{8} \mathrm{H}_{10}$ & 106.167 & 0.865 & 0.629 & 28.59 & 38.4 & $111.0 / 165.1$ \\
\hline m-Xylene & $\mathrm{C}_{8} \mathrm{H}_{10}$ & 106.167 & 0.861 & 0.559 & 28.26 & 37.9 & $106.0 / 174.0$ \\
\hline $\begin{array}{l}2- \\
\text { Chlorotoluene }\end{array}$ & $\mathrm{C}_{7} \mathrm{H}_{7} \mathrm{Cl}$ & 126.585 & 1.077 & 0.932 & 32.95 & $37.1^{[\mathrm{c}]}$ & n.d. / 106.3 \\
\hline
\end{tabular}



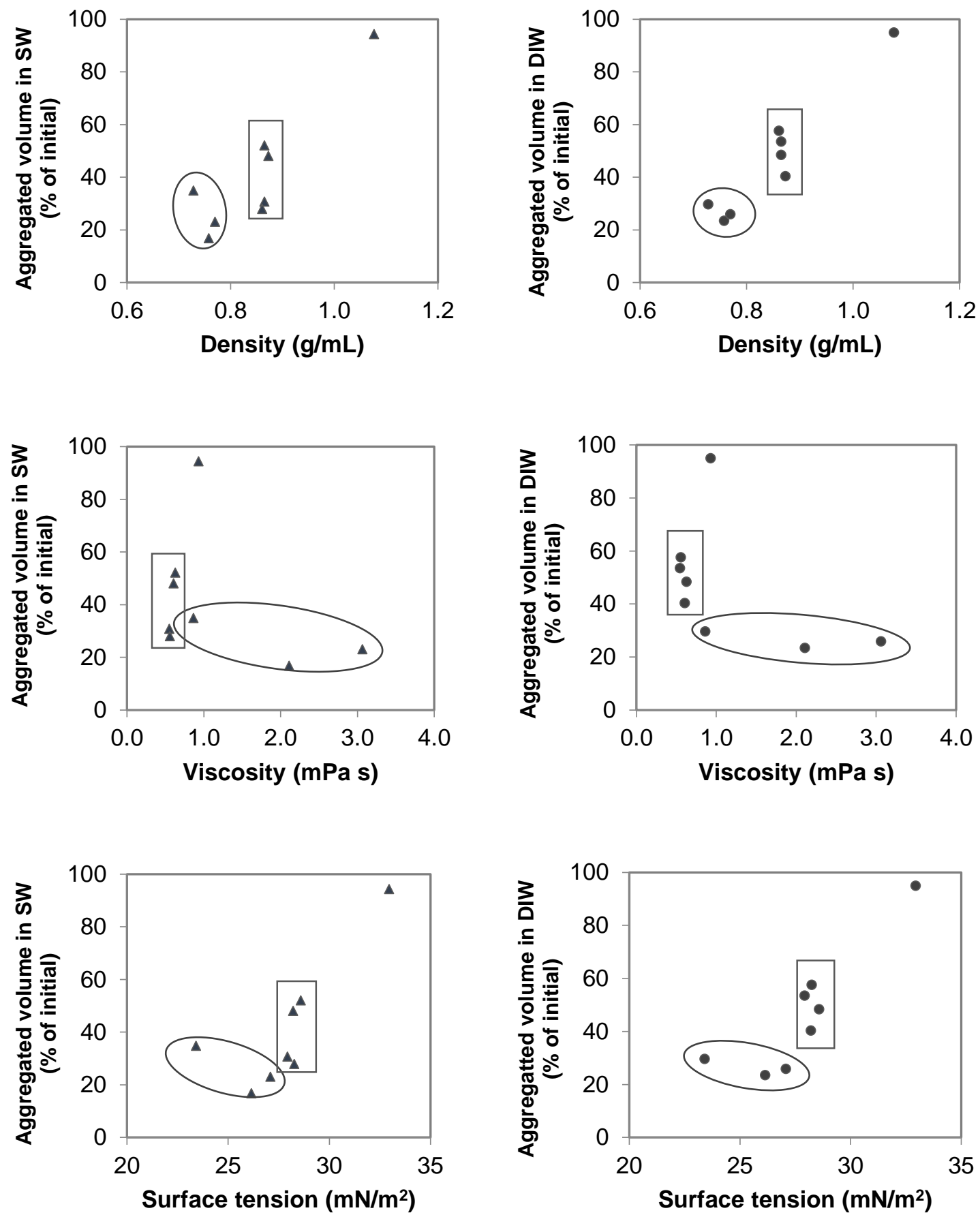

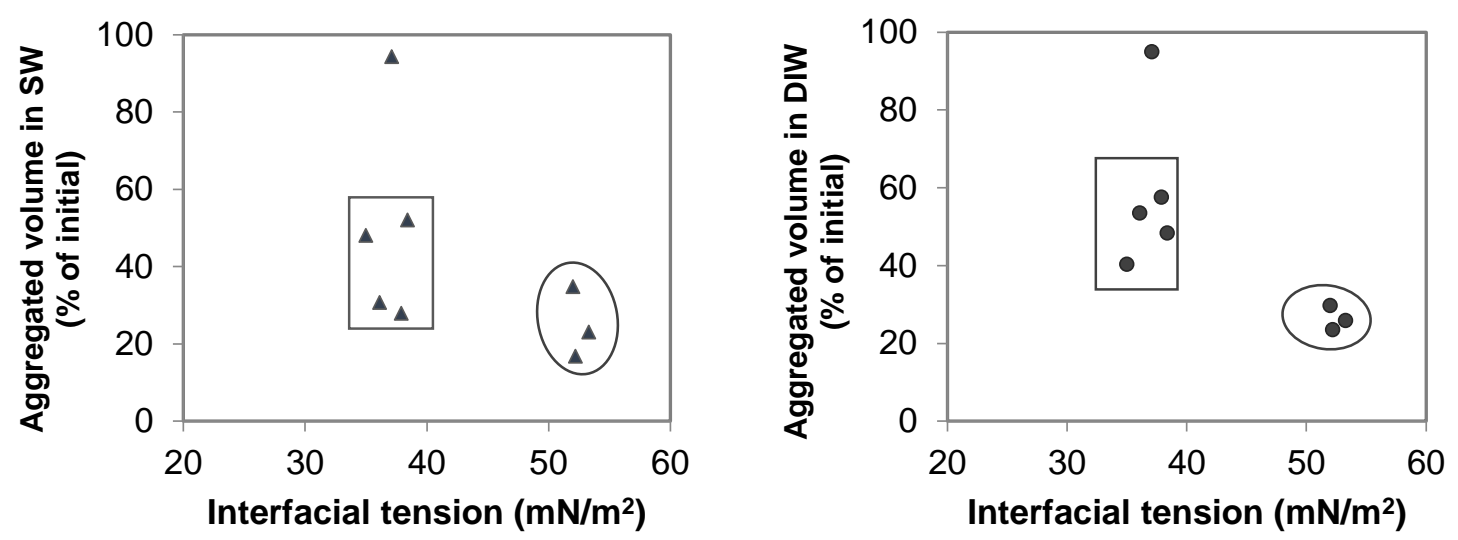

Figure 5.2. Volume of the aggregated HLs in salt water (SW) and deionized water (DIW) versus selected physical properties. Selection in ellipse indicates alkanes (decane, tetradecane, and hexadecane); in rectangle - BTEX group (benzene, toluene, ethylbenzene, and $\mathrm{m}$-xylene); 2-chlorotoluene is the one outside groupings.

Analysis of the data in Fig. 5.2 allowed us to develop formulas (1) and (2) that relate the amount of the aggregates to the properties of HLs. The aggregation number $\left(A_{N}\right)$ of a HL relative to the water properties was introduced for two cases:

1) Kinematic viscosity of the HL is lower than the kinematic viscosity of water $\left(n_{w}=10^{-6} \mathrm{~m}^{2} / \mathrm{s}\right) ;$ or $n_{H L}<n_{w}$, then:

$$
A_{N}=\frac{\rho_{H L} \gamma_{a i r} n_{w}}{\rho_{w} \gamma_{w} n_{H L}}
$$

2) Kinematic viscosity of a HL is higher than the kinematic viscosity of water $\left(n_{w}=10^{-6} \mathrm{~m}^{2} / \mathrm{s}\right)$ or $n_{H L}>n_{w}$, then:

$$
A_{N}=\frac{\rho_{H L} \gamma_{\text {air }}}{\rho_{w} \gamma_{w}} \log \frac{n_{H L}}{n_{w}}
$$

Mass and volume of the aggregated HL can be estimated by the following equations:

$$
M_{A}=A_{N} M_{H L}
$$




$$
V_{A}=A_{N} V_{H L}
$$

where $\rho_{H L}$ and $\rho_{w}$ are densities of a HL and water; $\gamma_{a i r}$ and $\gamma_{w}$ are surface tension of the $\mathrm{HL}$ and interfacial $\mathrm{HL}$ and water tension; $n_{H L}$ and $n_{w}$ are kinematic viscosities of a HL and water, respectively; and $M_{H L}$ is the mass of the floating $\mathrm{HL}, M_{H L}=V_{H L} \rho_{H L}$. Note that $M_{A}$ does not include mass of the adhered particles on the surface of the aggregates (globules).

Theoretical results and the experimentally observed data are presented in Fig. 5.3.
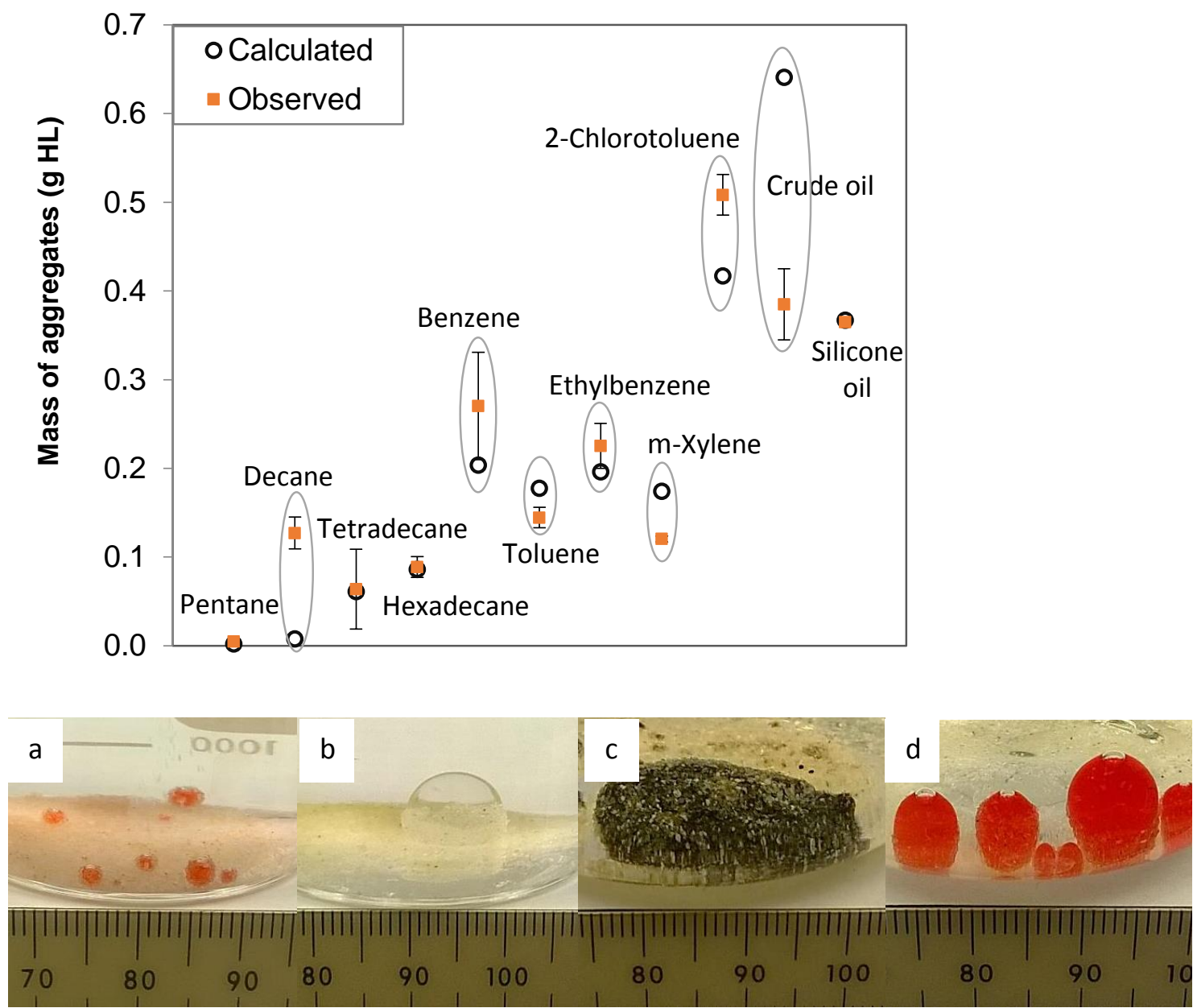

Figure 5.3. Observed and calculated mass of aggregates of the HLs in $\mathrm{SW}(0.5 \mathrm{~mL}$ of $\mathrm{HL}$ and $1 \mathrm{~g}$ of fine quartz sand; layer thickness to particle diameter ratio is > 4:1); error bars show \pm 1SD. a. Pentane; b. Silicone oil (viscosity $50 \mathrm{cSt}$ ); and c. Crude oil (South Louisiana crude) are added as additional HLs for validation of the equations 2-4. Among 
the eight initially tested HLs, d. 2-chlorotoluene showed the biggest range in size distribution (globule size) and it was the most stable in terms of its shape and captured volume in the globules (air bubbles are clearly visible on top of the globules).

Figure 5.4a summarizes the data collected on the behavior of the eight HLs over time (decrease in volume, \%) for the experiments conducted with deionized water and saltwater. The rate constants for the change in the aggregated volume (loss due to solubilization) were estimated for each HL in salt water and deionized water (Table 5.3, Fig. 5.4b).

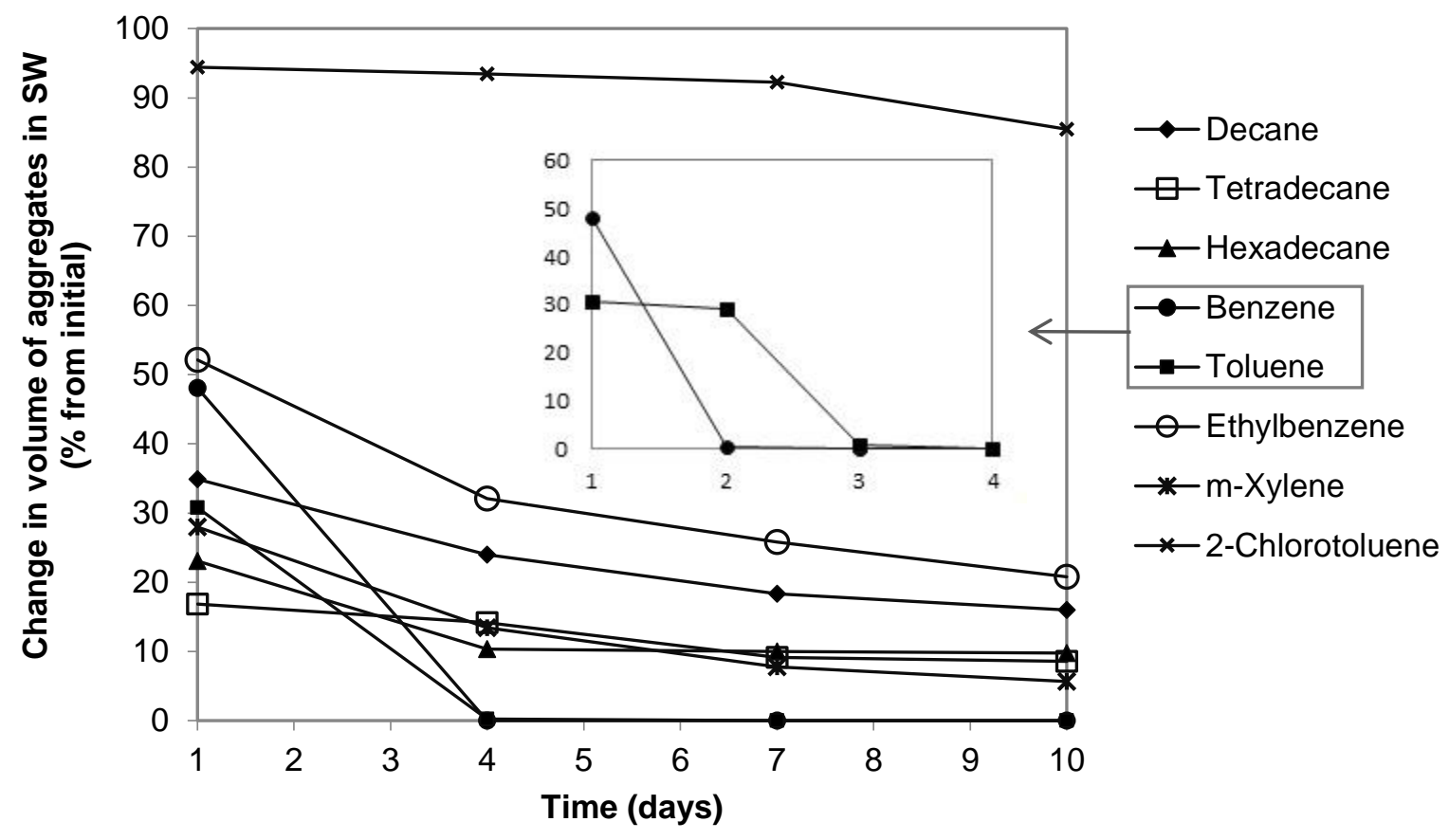




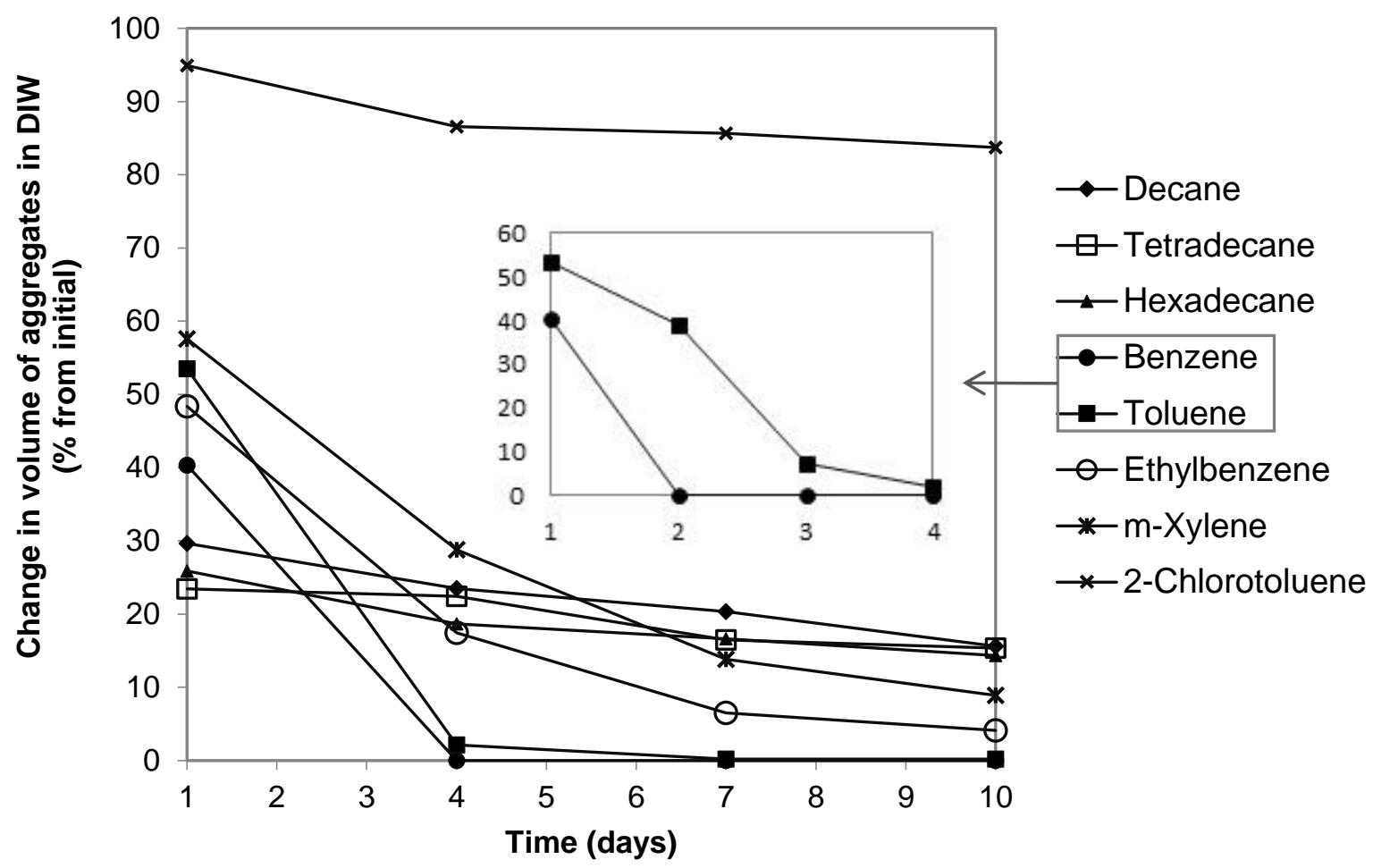

Figure 5.4a. Decrease in total volume of the aggregated globules of HLs during 10 days. Data are averages of two series of experiments conducted with salt water (SW) and two series in deionized water (DIW).
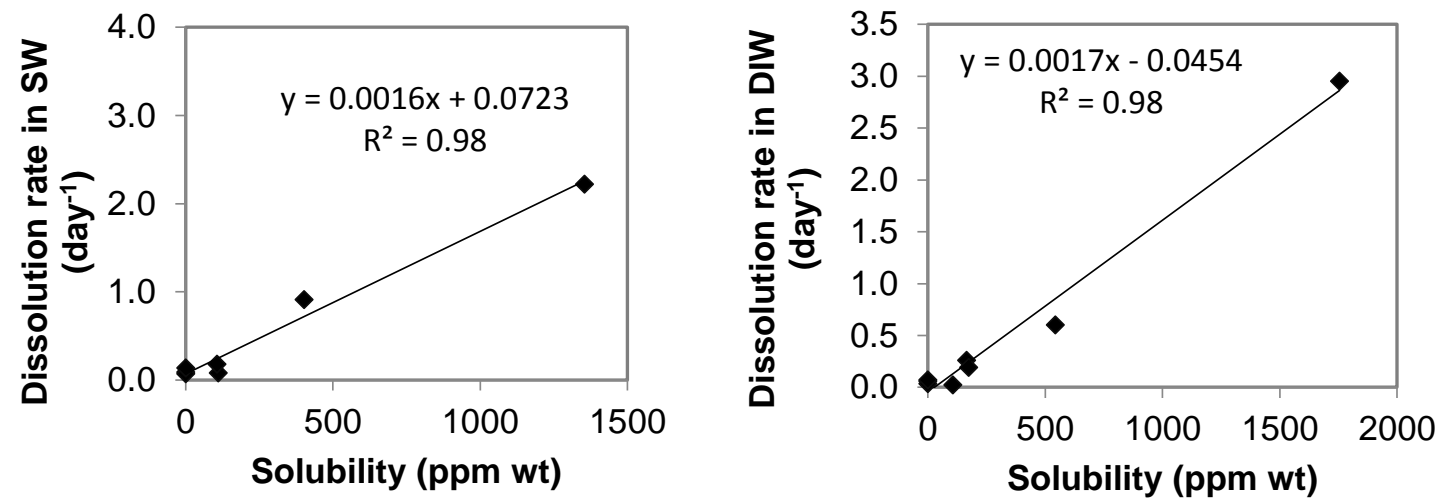

Figure 5.4b. Estimated dissolution rates in relation to solubility of the HLs.

Figure 5.4. Time dependency of the aggregated volume of globules and dissolution rates $\left(\right.$ day $\left.^{-1}\right)$ of the HLs. 
Table 5.3. Dissolution rate constants $\left(\mathrm{day}^{-1}\right)$ of the volume loss in aggregated globules of HL (time period $=10$ days).

\begin{tabular}{lll}
\hline Hydrophobic liquid & Salt water, day ${ }^{-1}$ & Deionized water, day ${ }^{-1}$ \\
\hline Decane & 0.09 & 0.06 \\
Tetradecane & 0.07 & 0.04 \\
Hexadecane & 0.14 & 0.07 \\
Benzene & 2.22 & 2.95 \\
Toluene & 0.91 & 0.60 \\
Ethylbenzene & 0.08 & 0.26 \\
m-Xylene & 0.18 & 0.19 \\
2-Chlorotoluene & 0.01 & 0.02 \\
\hline
\end{tabular}

\section{Discussion and Conclusion}

Experiments were conducted with HLs which formed certain thickness of the floating layer (layer thickness to particle diameter ratio was > 4:1) and a solid granular material (fine quartz sand, diameter $<0.425 \mathrm{~mm}$ and $>0.075 \mathrm{~mm}$ ). Under these conditions, we observed direct relationships between the amount of the HL aggregated as globules and HL density and surface tension (Fig. 5.2). Interfacial tension (HL and water) and viscosity showed nonlinear inverse correlations with the total volume the HL captured in the globules formed by sand addition. The data exhibited similar trends for salt water and deionized water (Fig. 5.2). The aggregation number $\left(A_{N}\right)$ (relative to water) was introduced to correlate the properties of HLs and the aggregation rates (by weight or by volume). The aggregation number was developed for two cases, depending on the kinematic viscosity of the HL (higher or lower than the viscosity of water, $n_{w}=1 \mathrm{cSt}$ ). Calculated values of the predicted aggregated mass of the HLs were plotted versus observed data (Fig. 5.3). In addition, three liquids were tested for validation of the equations derived. These included pentane $(n=0.038 \mathrm{cSt})$, crude South Louisiana oil $(n=11.7 \mathrm{cSt})$, and silicone oil $(n=$ 
$50 \mathrm{cSt}$ ). Pentane and silicone oil showed good agreement with the calculated values; however, the calculated value for the crude oil was higher than the predicted value (estimated as $0.64 \mathrm{~g}$ versus measured as $0.38 \mathrm{~g}$ ) obtained (data are averages of duplicate tests). This may be explained by the higher complexity of the crude oil as the mixtures of numerous organic compounds with different properties and polarities. Experimental aggregation data can also vary depending on the manner the granular material is applied (i.e., sprinkling vs all at once).

Once formed, globules of the aggregated HLs with fine quartz sand sank to the bottom of the Erlenmeyer flask. Globules were fully or partially covered by sand particles and had air bubbles (on top) due to entrapment of air during the rapid formation and sinking of the globules (Fig. 5.1d, 5.1f, 5.1g, 5.1h, and 5.3d). Some of fully sand covered globules (alkanes group, the lowest densities of the tested HLs) and partially sand covered globules (BTEX group, higher densities) slowly ascended to the water surface and collapsed (or floated) on the water surface after a few hours or days as the sand was released from the globule surface (Fig. 5.1d and 5.1f). The amount of the aggregated HLs in the globules was also altered over time, as the size of the globules decreased due to dissolution of HL from globules (Fig. 5.4a). Dissolution rate constants were found to be highly correlated with the solubility of HLs $\left(\mathrm{R}^{2}=0.98\right.$ for both salt water and deionized water, Fig. 5.4b). The globules formed with the most volatile and soluble HLs tested, benzene and toluene (dissolution rate constants up to $2.95 \mathrm{day}^{-1}$ and $0.91 \mathrm{day}^{-1}$, respectively), were monitored more frequently (every day during the first four days until they disappeared completely, with no aggregated form remaining) (Fig. 5.4a). The dissolution rates of some of the HLs 
were higher in the deionized water than those measured in salt water (Fig. 5.4a and Table 5.3), as expected from the solubility properties (Table 5.2).

As a potential environmentally friendly method for capturing floating oil slicks (light hydrophobic liquids) with natural granular materials (such as sand) can provide benefits for reducing the impact radius after spills of HLs in water, and developing controlled subsurface remediation methods for the captured HLs. Higher aggregation tendency was observed for the HLs with relatively high densities (but lower than the density water) and HLs with high surface tension. Aggregated HLs dissolved in water from the globules over time, depending on their solubility. In the case of a crude oil slick captured with sand, aromatic compounds such as benzene and toluene are likely to disappear from the aggregated form within the first 3-4 days. In order to capture and recover such compounds, the response time should be planned accordingly.

\section{Acknowledgments}

Partial funding for this research has been provided by Gulf of Mexico Research Initiative (GoMRI) through funding to Consortium for the Molecular Engineering of Dispersant Systems (C-MEDS).

\section{References}

Abkarian, M., Protiere, S., Aristoff, J.M., and Stone, H.A. 2013. Gravity-induced encapsulation of liquids by destabilization of granular rafts. Nature Communications, 4 (1895).

Atlantic, O.C.S. 1988. Synthesis of effects of oil on marine mammals. Geraci, J.R., Aubin, D.J.S. (Eds.), Department of the Interior, Minerals Management Service, Atlantic OCS Region. 
Bandara, U.C., Yapa, P.D., and Xie, H. 2011. Fate and transport of oil in sediment laden marine waters. Journal of Hydro-environmental Research, 5 (3): 145-156.

Boglaienko, D., and Tansel, B. 2015. Instantaneous stabilization of floating oils by surface application of natural granular materials (beach sand and limestone). Marine Pollution Bulletin, 91 (1): 107-112.

Bragg, J.R., and Owens, E.H. 1995. Shoreline cleansing by interactions between oil and fine mineral particles. Proc. Intl. Oil Spill Conf. Feb. 22-Mar 2 1995, Long Beach, CA, American Petroleum Institute, Washington, DC, 4620: 219-227.

Bragg, J.R., and Yang, S.H. 1995. Clay-oil flocculation and its effects on the rate of natural cleansing in Prince William Sound following the Exxon Valdez oil spill, in: Exxon Valdez Oil Spill - Fate and Effects in Alaskan Waters; Wells, P.G., Butler, J.N., Hughes, J.S. (Eds.), American Society for Testing and Materials, Philadelphia, PA. ASTM STP, 1219, 178-214.

Demond, A.H., and Lindner, A.S. 1993. Estimation of interfacial tension between organic liquids and water. Environmental Science \& Technology, 27: 2318-2331.

Gong, Y., Zhao, X., Cai, Z., O'Reilly, S.E., Hao, X., and Zhao, D. 2014. A review of oil, dispersed oil and sediment interactions in the aquatic environment: influence on the fate, transport and remediation of oil spills. Marine Pollution Bulletin, 79 (1-2): 1633.

Guyomarch, J., Le Floch, S., and Merlin, F.-X. 2002. Effect of suspended mineral load, water salinity and oil type on the size of oil-mineral aggregates in the presence of chemical dispersant. Spill Science \& Technology Bulletin, 8 (1): 95-100.

Hunter, R.J. 2001. Foundations of colloid science. Oxford University Press.

Israelachvili, J.N. 1985. Intermolecular and surface forces: With applications to colloidal and biological systems. Academic press: New York.

Jasper, J.J., and Seitz, H.R. 1958. The temperature-interfacial tension studies of some halogenated toluenes against water. The Journal of Physical Chemistry, 62 (10): 1331-1333.

Khelifa, A., Stoffyn-Egli, P., Hil, P.S., and Lee, K. 2002. Characteristics of oil droplets stabilized by mineral particles: effects of oil type and temperature. Spill Science \& Technology Bulletin, 8 (1): 19-30.

Lee, K. 2002. Oil-particle interactions in aquatic environments: influence on the transport, fate, effect and remediation of oil spills. Spill Science \& Technology Bulletin, 8 (1): 3-8. 
Muschenheim, D.K., and Lee, K. 2002. Removal of oil from the sea surface through particulate interactions: review and prospectus. Spill Science \& Technology Bulletin, 8 (1): 9-18.

Payne, R.J., Clayton, Jr. J.R., and Kirstein, B.E. 2003. Oil/Suspended particulate material interactions and sedimentation. Spill Science \& Technology Bulletin, 8 (2): 201221.

Schwarzenbach, R.P., Gschwend, P.M., and Imboden, D.M. 1993. Environmental organic chemistry. John Wiley \& Sons.

Sørensen, L., Melbye, A.G., and Booth, A.M. 2014. Oil droplet interaction with suspended sediment in the seawater column: influence of physical parameters and chemical dispersants. Marine Pollution Bulletin, 78: 146-152.

Sterling, M.C., Bonner, J.S., Page, C.A., Fuller, C.B., Ernest, A.N.S., and Autenrieth, R.L. 2004. Modeling crude oil droplet-sediment aggregation in nearshore waters. Environmental Science \& Technology, 38 (17): 4627-4634.

Stoffyn-Egli, P., and Lee, K. 2002. Formation and characterization of oil-mineral aggregates. Spill Science \& Technology Bulletin, 8 (1): 31-44.

Wood, P.A., Lunel, T., Daniel, F., Swannell, R., Lee, K., and Stoffyn-Egli, P. 1998. Influence of oil and mineral characteristics on oil-mineral interaction. Proceedings of the twenty-first Arctic and marine oil spill program (AMOP) technical seminar. Environment Canada, Ottawa, Ont., 51-77.

Yaws, C.L. 1999. Chemical properties handbook: physical, thermodynamic, environmental, transport, safety, and health related properties for organic and inorganic chemicals. McGrown-Hill. 


\title{
CHAPTER VI
}

\section{GRAVITY INDUCED DENSIFICATION OF FLOATING CRUDE OIL BY \\ GRANULAR MATERIALS: EFFECT OF PARTICLE SIZE AND SURFACE MORPHOLOGY}

Boglaienko, D., and Tansel, B. 2016. Science of The Total Environment, 556: 146-153.

DOI: 10.1016/j.scitotenv.2016.01.214

\begin{abstract}
Densification and sedimentation of floating crude oil to the bottom of water column reduces the radius of a spill and its mobility, preventing direct contamination of beaches, coastal flora and fauna. Performances of different natural granular materials were evaluated for capturing efficiency of floating fresh South Louisiana crude oil. The granular materials studied were quartz sand with medium (20-30 mesh) and fine (40-100 mesh) particle size, limestone with coarse (4-10 mesh) and medium (16-40 mesh) particle size, beach sand (2080 mesh), and clay (kaolin with ferric oxide; passing 200 mesh). Beach sand (mixture of quartz and limestone 20-80 mesh) and limestone (16-40 mesh) demonstrated better performance for capture, densification and submergence of the crude oil among the materials evaluated. The behavior of granular particles with the hydrophobic phase can be classified as (1) immersion entrapment inside the hydrophobic phase, and (2) partial encapsulation of the hydrophobic phase by a single layer of particles. With crude oil, the particles were primarily entrapped within the hydrophobic phase. Study of the effect of particle size and morphology (i.e., porosity) of the granular materials on capture performance showed that average surface pore size did not have a significant effect on
\end{abstract}


aggregation with oil, however, higher capture efficiency was observed with materials of higher surface porosity (beach sand and limestone). The experiments revealed that there is a critical particle size range (passing 10 mesh) which resulted in more effective aggregation of the granular materials with crude oil.

\section{Introduction}

The World oil production has increased from 8.5 million tons in 1985 to 11.7 million tons in 2000 in parallel with the number of offshore platforms also increased from a few thousand in 1985 to about 8,300 in 2000 (Kvenvolden and Cooper, 2003; Input of Oil, 2003). The amount of oil entering marine waters globally is estimated at 1.3 million tons per year with 0.6 million tons from natural seeps, 0.150 due to spills during transportation, and 0.450 million tons from spills during consumption of petroleum (Input of Oil, 2003). Oil spills at sea have significant impacts in both open seas and near shore environments due to spreading behavior of the floating oil (Tansel, 2014). The effects of a petroleum release depend on the rate of release, characteristics of the petroleum, and the local physical and biological character of the exposed ecosystems (Input of Oil, 2003). Fresh spills of crude or petroleum based oils at sea move with currents and mixing due to wind and sea conditions, quickly enlarging the impact radius. Spills near coastal areas effect the ecosystems that are nesting grounds of both land and sea organisms as well as economically important marine resources (e.g., fish, shrimp beds, beaches) (Tansel et al., 2013; Tansel et al., 2014). For large oil spills at sea, the most commonly used response method is the dispersant application by spraying. However, dispersants, as primary response method for oil spills, have limitations for use near coastal areas and can be applied 
in areas farther than $5.6 \mathrm{~km}$ (or 3 nautical miles) from shore and in waters deeper than 30 feet (or 10 m) (Making Decisions, 2005; Dispersant Use, n.d.).

Transport of oil to sediments is considered as important natural removal mechanism (Payne et al., 1987; Bandara et al., 2011; Gong et al., 2014) and is called 'surf washing' (Lee, 2002; Owens and Lee, 2003; Sterling et al., 2004). In coastal areas, crude oil has a tendency to form clay-oil flocs that add to shoreline cleansing (Bragg and Owens, 1995). It was proposed to accelerate clay-oil flocculation process via spreading the mineral fines (clays) on the shoreline (Bragg and Yang, 1996). Overall, it was summarized that between 20 to $30 \%$ of the total amount spilled can be naturally submerged and transported to the sea bed as clay-oil flocs (Muschenheim and Lee, 2002); the other scenario simulations go up to $65 \%$ of removed oil in the aggregated form with sediments (Bandara et al., 2011).

Our previous studies showed that capture (and densification) and aggregation efficiency of fresh South Louisiana crude oil with granular materials can be as much as $80 \%$ when dry granular materials (i.e., limestone or quartz sand) are applied directly on the floating oil slick (Boglaienko and Tansel, 2015; Boglaienko et al., 2016). When granular particles are applied to the floating oil layer, they contact with the oil phase first, hence, become hydrophobic before reaching the water phase. This is a significantly different process from the above mentioned studies, and allows achieving much higher efficiency for aggregation and capture of floating oil. The granular particle induced submergence of floating oil, referred as raft formation (as a special case) by granular particles, was also identified as a potential oil spill remediation method by Abkarian et al. (2013) for silicone oil or mineral oils. Estimation of the amount of granular material ( $\mathrm{kg}$ or $\mathrm{lbs}$ ) needed to 
capture a fresh oil spill of a certain volume (liters or barrels) was performed by Boglaienko and Tansel (2015) based on the experiments conducted with South Louisiana crude oil.

Sedimentation of floating crude oil to the bottom of water column does not eliminate the toxic compounds from being released to the environment, but reduces their mobility (i.e., keeping the impact radius small) and prevents direct contamination of beaches, birds, and coastal flora. The objectives of this study were to compare the performance of different natural granular materials for capturing floating crude oil in relation to their particle size and surface morphology. Based on the experimental results, we evaluated the differences in granular aggregation efficiencies of different natural granular materials with crude oil.

\section{Materials and Methods}

South Louisiana crude (SLC) oil was obtained from the BP America Production Company (Houston, TX). Quartz sand with medium particle size (20-30 mesh) was obtained from Spectrum Chemical MFG. Quartz sand with fine particle size (40-100 mesh) was obtained from Acros Organics. Limestone with medium particle size (16-40 mesh), beach sand (20-80 mesh), and clay (kaolin with ferric oxide; passing 200 mesh) were classified by sieving using standard sieves. The geophysical characteristics of the granular materials are presented in Table 6.1.

Bulk density was measured as the ratio of dry mass to bulk volume, using a $25-\mathrm{mL}$ graduated cylinder and an analytical balance. Particle surfaces were examined by Scanning Electron Microscopy (SEM) in combination with the qualitative analysis by Energy Dispersive Spectroscopy (Field Emission SEM JEOL 6330 with EDS “Thermo"). Pore 
sizes were measured during the SEM imaging; and specific pore surface area was estimated with ImageJ 1.48v software (National Institutes of Health, USA).

Aggregation experiments were performed in 125-mL Erlenmeyer flasks filled with $100 \mathrm{~mL}$ tap water and $0.5 \mathrm{~mL}$ fresh SLC oil. For the experiments, $1.00 \pm 0.01 \mathrm{~g}$ granular material was applied directly to the floating crude oil. Experiments conducted with different amounts of granular material showed that this was the most effective granular material to oil ratio for the materials used (Boglaienko and Tansel, 2015). The thickness of the oil slick was $0.7 \mathrm{~mm}$ on average.

Table 6.1. Granular materials: particle size and bulk density; composition by Energy Dispersive Spectroscopy (EDS) and images by Scanning Electron Microscopy (SEM): X15 for medium quartz sand, fine quartz sand, and beach sand; X20 for limestone and clay.

\begin{tabular}{|c|c|c|c|c|}
\hline Material & $\begin{array}{l}\text { Mesh size / } \\
\text { opening, } \\
\text { mm }\end{array}$ & $\begin{array}{l}\text { Bulk } \\
\text { density, } \\
\mathrm{g} / \mathrm{cm}^{3}\end{array}$ & $\begin{array}{l}\text { Composition, } \\
\text { weight } \%\end{array}$ & Image $^{[1]}$ \\
\hline $\begin{array}{l}\text { Medium } \\
\text { quartz sand }\end{array}$ & $\begin{array}{l}20-30 / \\
0.85-0.60\end{array}$ & 1.68 & $\begin{array}{l}\text { Si: } 54.12 \\
\text { O: } 45.88\end{array}$ & \\
\hline $\begin{array}{l}\text { Fine quartz } \\
\text { sand }\end{array}$ & $\begin{array}{l}40-100 / \\
0.42-0.15\end{array}$ & 1.56 & $\begin{array}{l}\text { O: } 51.98 \\
\text { Si: } 48.02\end{array}$ & \\
\hline
\end{tabular}




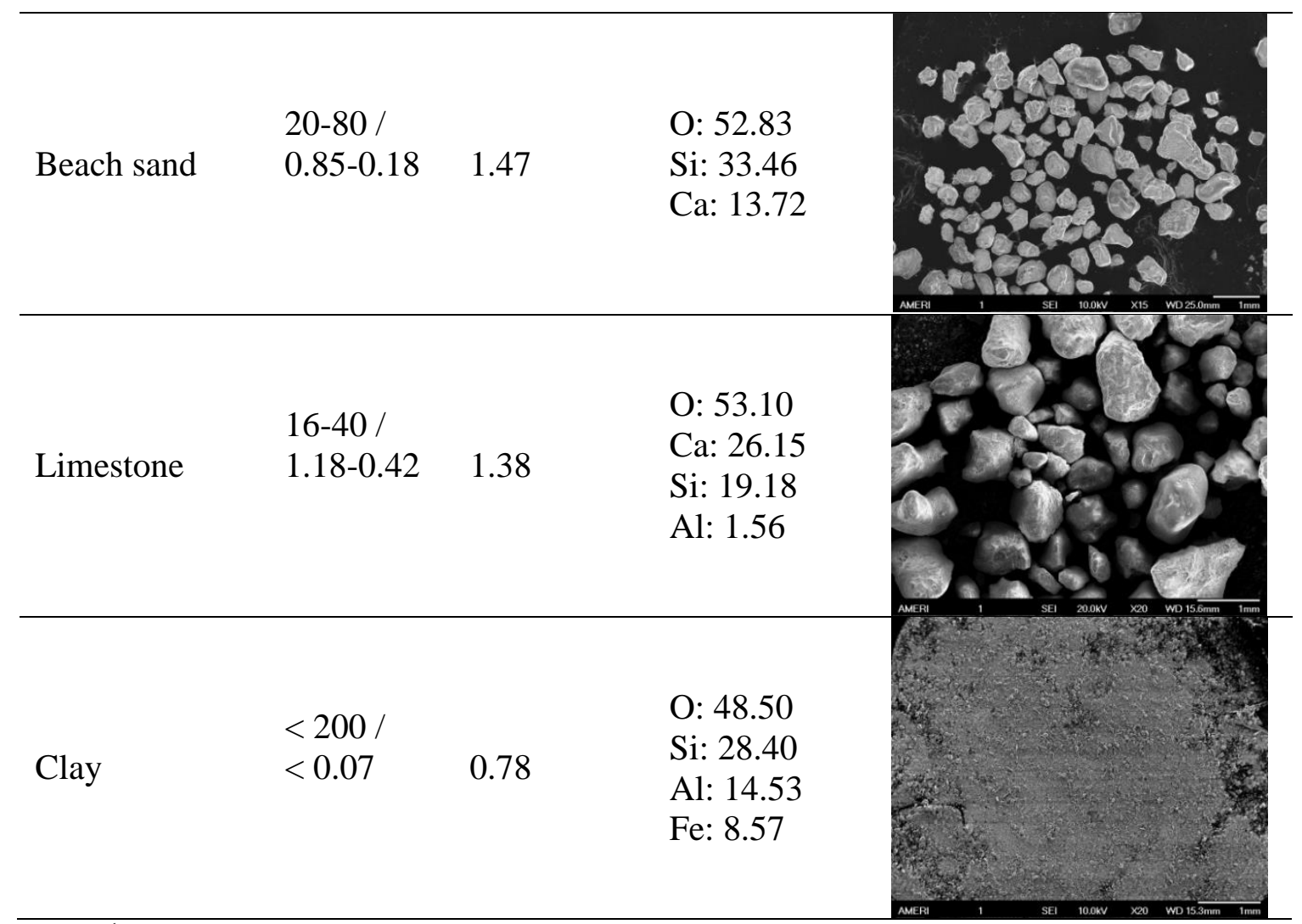

${ }^{1}$ Scale bar: $1 \mathrm{~mm}$

Preliminary tests showed that application rate of the granular material (quickly or slowly by sprinkling) made a noticeable difference in the aggregation and submergence of the floating oil with granular particles. Preliminary experiments were conducted to evaluate the effect of different rates of sand addition to the floating oil layer. Experimental observations showed that fast sand addition was more effective than slow addition (i.e., sprinkling) for submerging the floating oil. We have performed multiple tests which showed good repeatability of the results, when sand was preweighted and added to the oil layer from about $6 \mathrm{~cm}$ distance from the oil surface rapidly (using a small container which is enough to hold the sand). For the experiments, the granular material was weighed in a 
small container and applied all at once to the oil surface from about $6 \mathrm{~cm}$ distance (without mixing).

\section{Results}

Figures $6.1 \mathrm{a}$ and $6.1 \mathrm{~b}$ present the aggregation of SLC oil with different granular materials (6.1a floating oil, and $6.1 \mathrm{~b}$ close up of the submerged aggregate). Coarse limestone (4-10 mesh; 4.75-2.00 mm) was added to study the effect of particle size (Fig. 6.1a). However, coarse limestone did not aggregate well with crude oil. Hence, it was considered as ineffective and not included in Fig. 6.1b. Images in Fig. 6.1a are presented in the order from finer to coarser particle size.

Figure 6.2 presents the SEM images of the five granular materials studied at X6000 magnification. Medium sand (a), quartz sand (b) and beach sand (c) had relatively smooth surfaces. Limestone (d) has softer and more porous characteristics. Clay (e) particles exhibited light and fluffy characteristics and remained floating on the water surface for a couple of minutes.

Table 6.2 compares the characteristics of the granular particles with their oil capture potential. The residual oil remaining was quantified from the film thickness by developing a visual calibration scale. The calibration was performed by preparing a series of Erlenmeyer flasks (10 flasks with same size used in experiments) and adding different amounts of crude oil. The visual comparison of the residual oil with the calibration system allowed determination of the amount of residual oil. This was the best method to quantify the residual oil remaining as it was difficult to transfer the liquids due to smearing of crude

oil to the container resulting in loss of floating material. Beach sand was the most effective 
granular material for capturing floating oil. Beach sand also had the largest pores on the surface as well as the largest specific pore surface area measured as $\mathrm{nm}^{2}$ pores $/ \mathrm{nm}^{2}$ total surface. Fine quartz sand was the least effective and had the smallest specific pore surface area. Relationships between specific pore surface area, pore size (nm) and ratio of captured oil to the granular material $(\mathrm{mL} / \mathrm{g})$ are shown in Fig. 6.3.

The densification behavior of floating crude oil with granular materials is significantly different from that with pure hydrophobic liquids. Figure 6.4 compares the differences in the aggregation characteristics of a pure hydrophobic liquid (decane) of the alkane group (alkanes are the major component of the crude oil) and crude oil as a complex mix of hydrocarbons. 


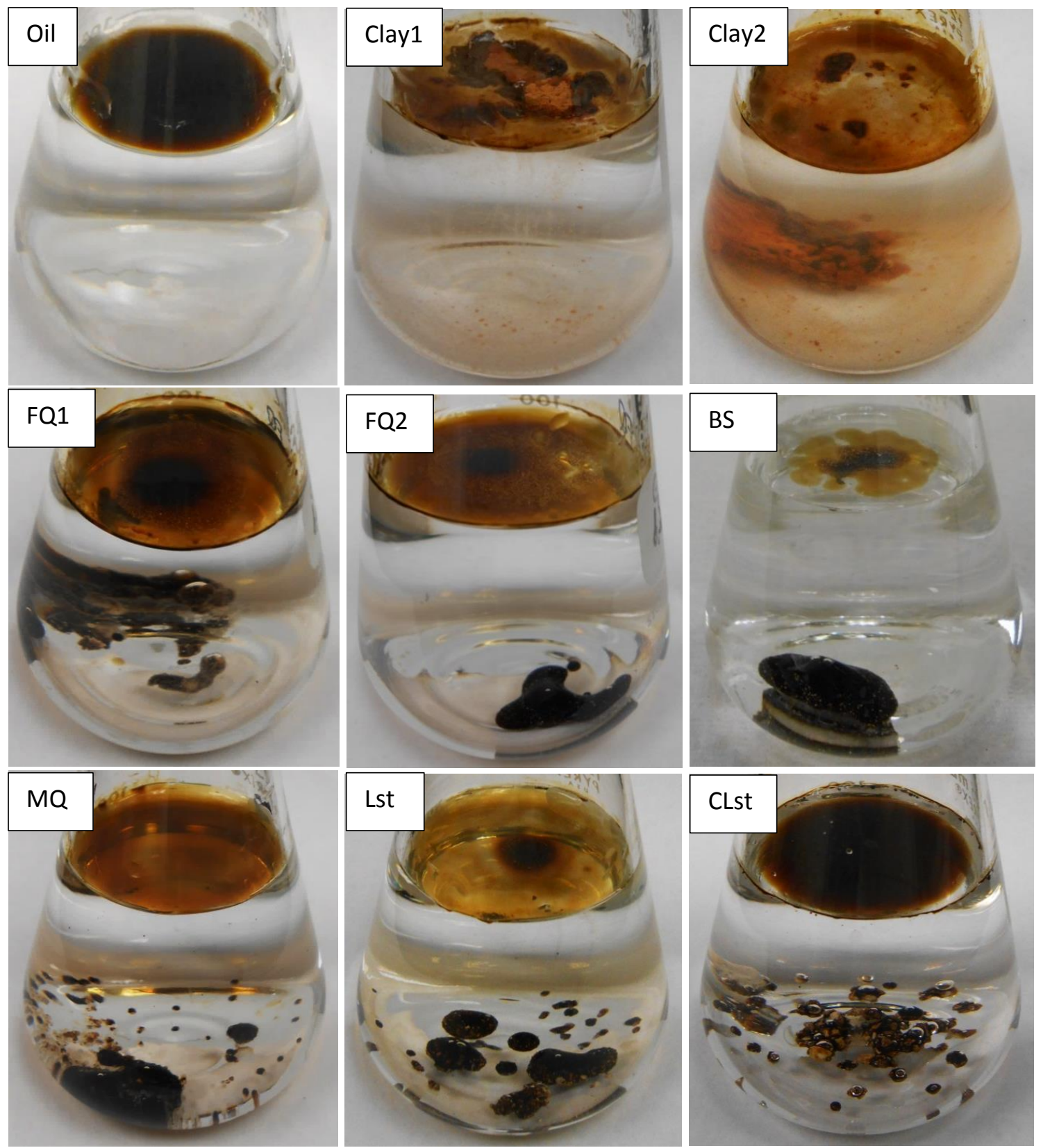

Figure 6.1a. Profile views of the aggregation of crude oil and different materials: Oil as the control; Clay1: right upon clay application; Clay2: 1.5 min after the application; FQ1: fine quartz sand, slow addition; FQ2: fine quartz sand, fast addition; BS: beach sand; MQ: medium quartz sand; Lst: limestone; and CLst: coarse limestone. 

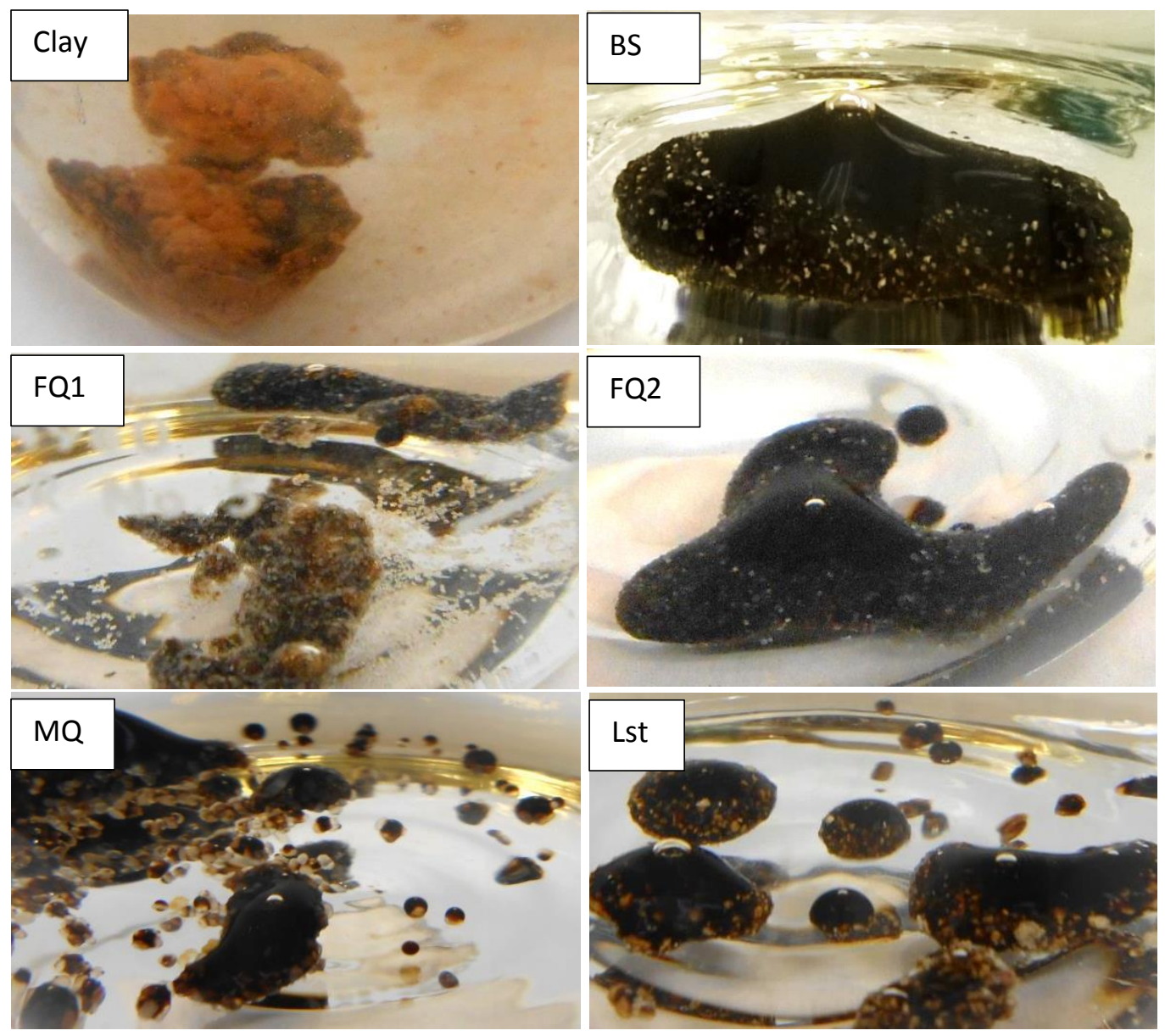

Figure 6.1b. Close up views on the aggregated materials with crude oil (bottom of a flask). Clay; BS: beach sand; FQ1: fine quartz sand, slow addition; FQ2: fine quartz sand, fast addition; MQ: medium quartz sand; and Lst: limestone.

Figure 6.1. Aggregation of SLC oil and different granular materials. 

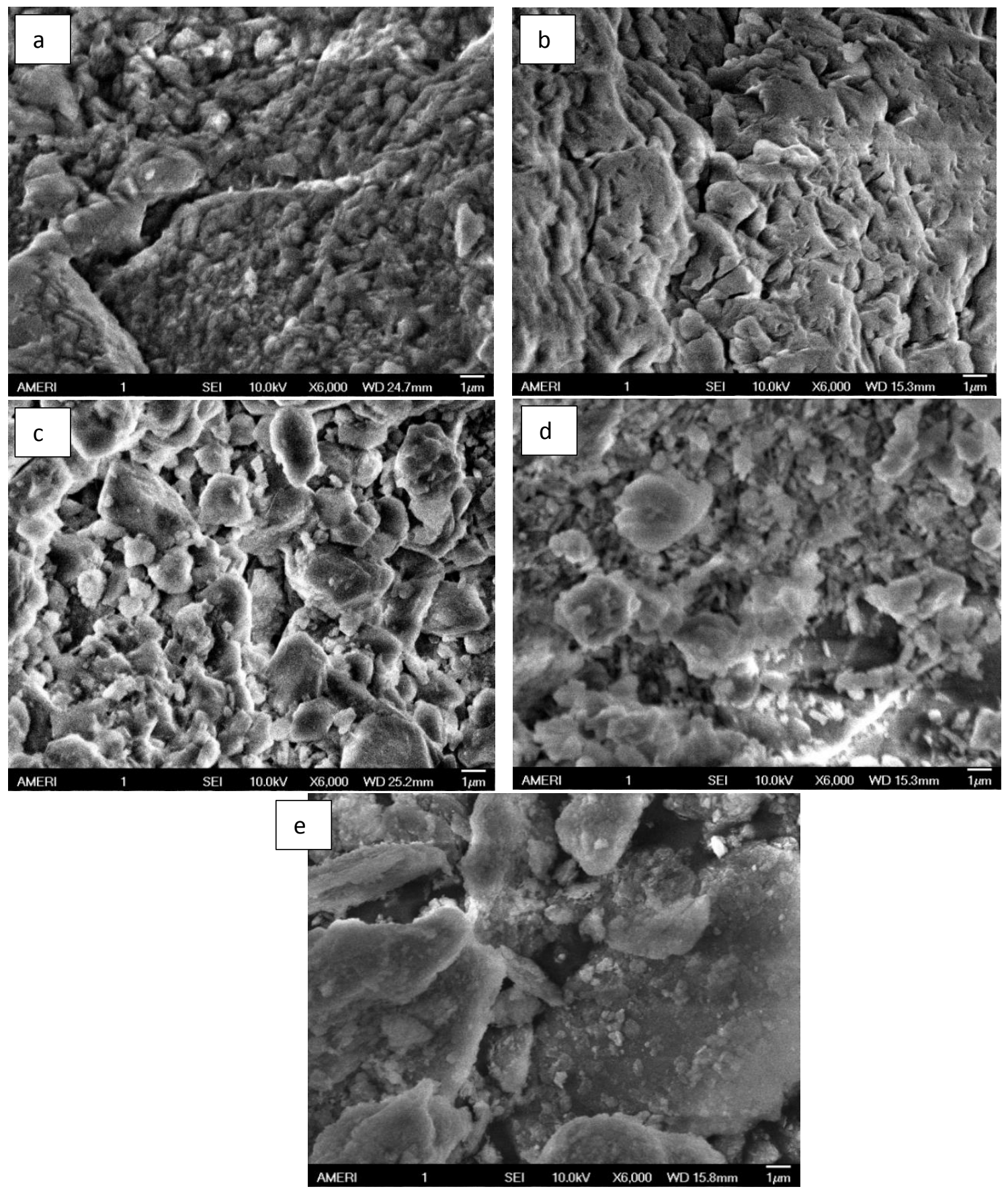

Figure 6.2. SEM images for the granular materials observed at the magnification X6000 (scale bar corresponds to $1 \mu \mathrm{m}$ ): a. Medium quartz sand; b. Fine quartz sand; c. Beach sand; d. Limestone; and e. Clay. 
Table 6.2. Porosity analyses of the granular materials (SEM - ImageJ) and aggregation ranking.

\begin{tabular}{|c|c|c|c|c|c|c|}
\hline Granular material & $\begin{array}{l}\text { Average } \\
\text { surface pore } \\
\text { size (St. } \\
\text { Dev.), nm }\end{array}$ & $\begin{array}{l}\text { Specific pore } \\
\text { surface area, } \\
\mathrm{nm}^{2} \text { pores / } \\
\mathrm{nm}^{2} \text { total } \\
\text { surface }\end{array}$ & $\begin{array}{l}\text { Granular } \\
\text { material to } \\
\text { oil } \\
\text { volumetric } \\
\text { ratio }\end{array}$ & $\begin{array}{l}\text { Captured } \\
\text { oil to } \\
\text { granular } \\
\text { material } \\
\text { ratio, } \\
\mathrm{mL} / \mathrm{g}\end{array}$ & $\begin{array}{l}\text { Floating } \\
\text { oil } \\
\text { remai- } \\
\text { ning, \% }\end{array}$ & $\begin{array}{l}\text { Rank } \\
\text { order of } \\
\text { agrega- } \\
\text { tion } \\
\text { effective } \\
\text {-ness } \\
\text { [1] }\end{array}$ \\
\hline $\begin{array}{l}\text { Medium quartz } \\
\text { sand }\end{array}$ & $208.7(115)$ & 0.12 & 1.18 & 0.30 & 40 & 4 \\
\hline Fine quartz sand & $482.8(402)$ & 0.11 & 1.28 & 0.12 & 75 & 5 \\
\hline Beach sand & 449.9 (329) & 0.31 & 1.36 & 0.45 & 10 & 1 \\
\hline Limestone & $348.4(195)$ & 0.18 & 1.44 & 0.40 & 20 & 2 \\
\hline Clay & $225.0(116)$ & 0.17 & 2.56 & 0.37 & 25 & 3 \\
\hline
\end{tabular}
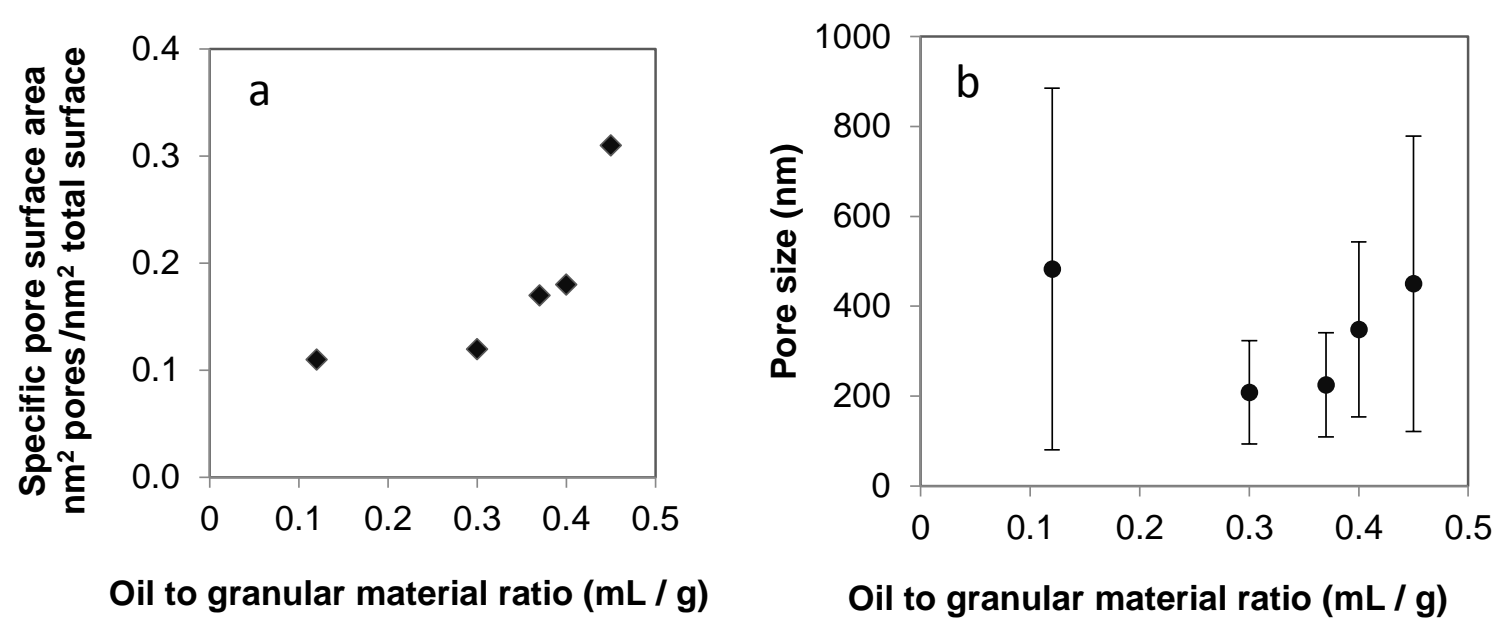

Figure 6.3. Variation of oil capture efficiency in relation to specific pore surface area (a) and pore size (b). 

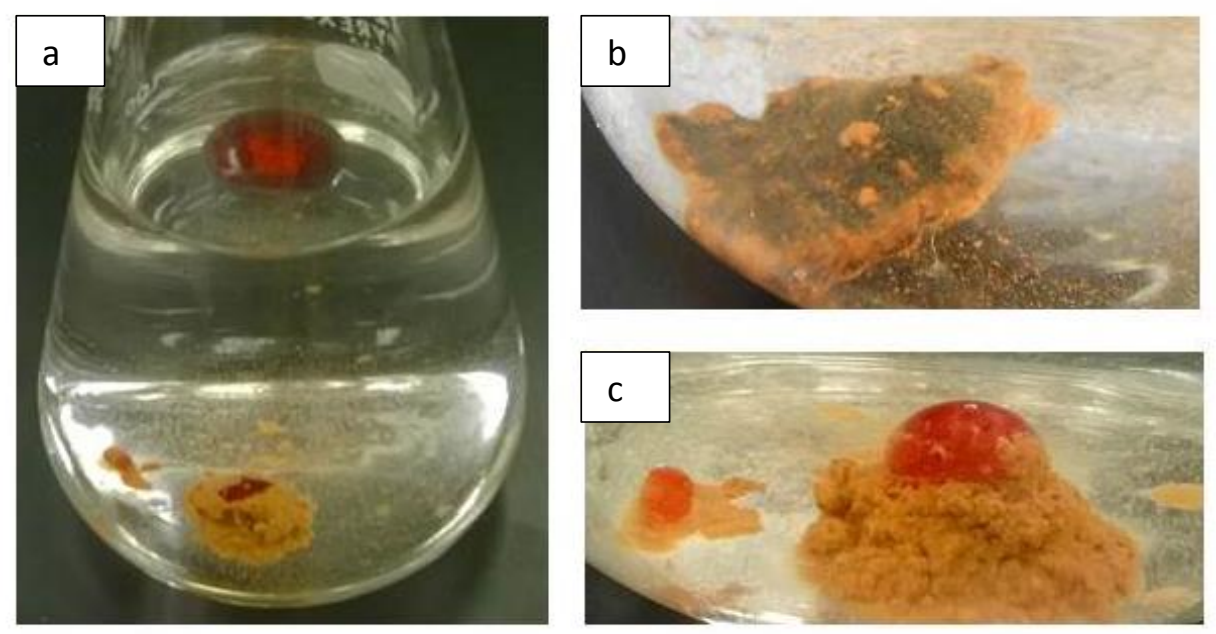

Figure 6.4. Comparison of aggregation of decane (dyed with Sudan IV) and SLC with clay: a. Decane immediately after clay addition; b. SLC oil after clay addition; and c. Decane 3 minutes after clay addition.

\section{Discussion}

Effectiveness of different types of granular materials were evaluated experimentally for capturing floating crude oil. The practical importance of the results provides a novel method for immobilizing floating crude oil after spills at sea. Differences in the aggregation charcateristics of the granular materials with SLC oil were observed and presented in Fig. 6.1, as the comparison of visual data.

Interaction of granular materials with floating hydropbic liquids (HL) results in formation of aggregates which are significantly different from those that form with pure HL as presented in Fig. 6.5. 


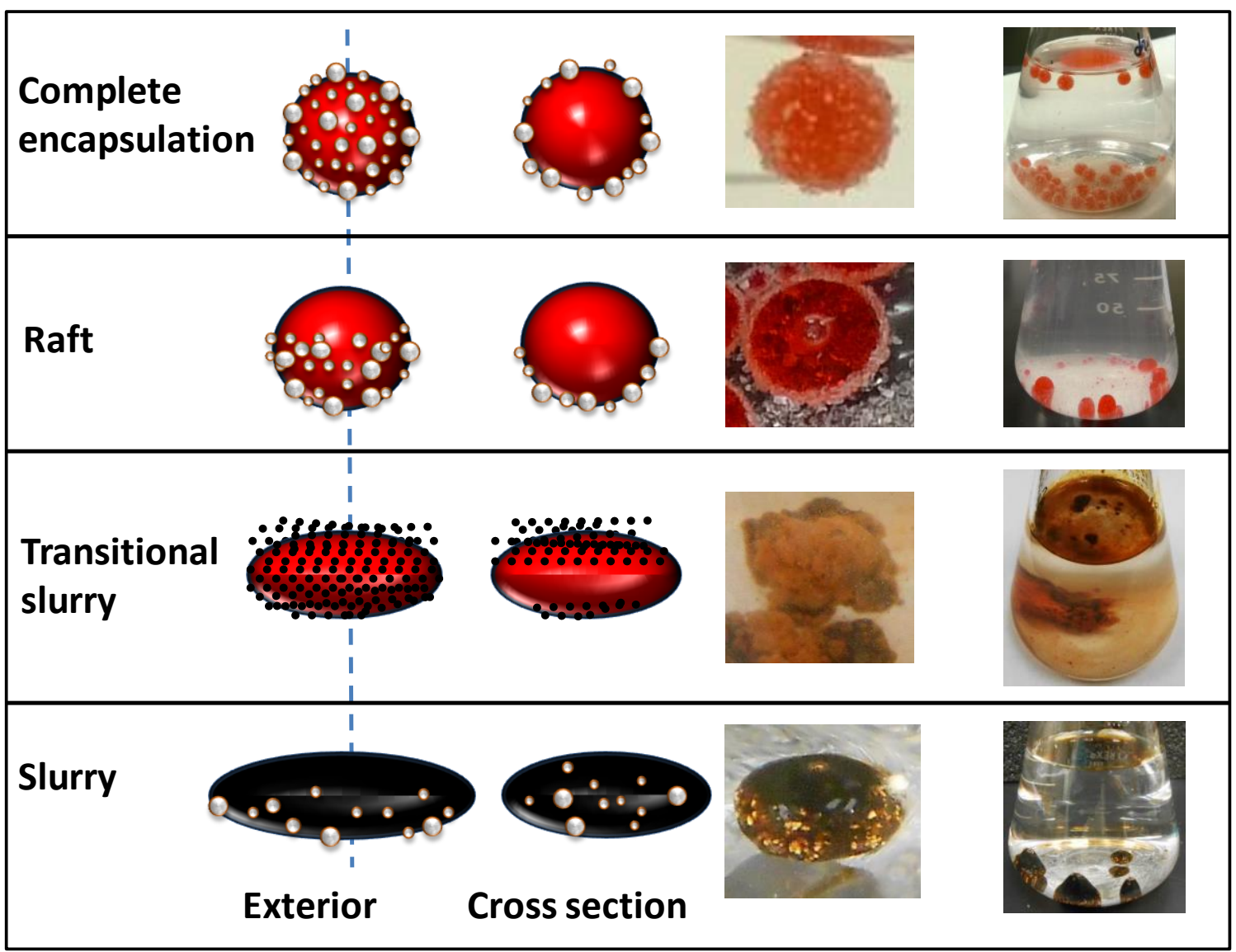

Figure 6.5. Different types of aggregates formed with granular particles and hydrophobic liquids. Complete encapsulation formed with a mixture of alkanes (decane, tetradecane, and hexadecane) and beach sand particles, raft formed with 2-chlorotoluene and beach sand particles, transitional slurry formed with SLC and clay, and slurry formed with limestone. 
Table 6.3. Comparison of experimental conditions in studies on submerging floating oils with granular particles with raft and slurry formation.

\begin{tabular}{|c|c|c|c|c|}
\hline \multirow{3}{*}{$\begin{array}{l}\text { System parameters } \\
\text { studied }\end{array}$} & \multicolumn{4}{|c|}{ Type of HL-granular particle aggregate formed } \\
\hline & $\begin{array}{c}\text { Raft } \\
\text { Slurry }{ }^{[1]}\end{array}$ & Raft & Raft & $\begin{array}{c}\text { Slurry } \\
\text { Transitional slurry }\end{array}$ \\
\hline & $\begin{array}{l}\text { Abkarian et al. } \\
2013\end{array}$ & $\begin{array}{c}\text { Tavacoli et al. } \\
2012\end{array}$ & $\begin{array}{c}\text { Boglaienko et al. } \\
2016\end{array}$ & This study \\
\hline \multicolumn{5}{|l|}{$\begin{array}{l}\text { 1. Hydrophobic } \\
\text { liquid }\end{array}$} \\
\hline - Type & Pure HLs & Pure HLs & Pure HLs & Crude oil \\
\hline $\begin{array}{l}\text { - Density, } \\
\mathrm{kg} / \mathrm{m}^{3}\end{array}$ & $\begin{array}{l}838 \text { (mineral oil) } \\
970 \text { (silicone oil) }\end{array}$ & $\begin{array}{l}\text { Considered for } \\
\text { dodecane (not } \\
\text { provided) } \\
\text { Considered for } \\
\text { octane (not } \\
\text { provided) }\end{array}$ & $\begin{array}{l}728 \text { (decane) } \\
758 \text { (tetradecane) } \\
770 \text { (hexadecane) } \\
873 \text { (benzene) } \\
865 \text { (toluene) } \\
865 \\
\text { (ethylbenzene) } \\
861 \text { (m-xylene) } \\
1077 \text { (2-chloro } \\
\text { toluene) }\end{array}$ & $\begin{array}{l}856 \text { (South } \\
\text { Louisiana crude) }{ }^{[5]}\end{array}$ \\
\hline $\begin{array}{l}\text { - Interfacial } \\
\text { tension } \\
\text { (oil-water), } \\
\mathrm{mN} / \mathrm{m}\end{array}$ & $\begin{array}{l}51 \text { (mineral oil) } \\
30 \text { (silicone oil) }\end{array}$ & Considered & - & $\begin{array}{l}\text { Experimental } \\
\text { observations } \\
\text { showed this to be } \\
\text { an important } \\
\text { parameter }\end{array}$ \\
\hline $\begin{array}{l}\text { - Surface } \\
\text { tension } \\
\text { (oil-air), } \\
\mathrm{mN} / \mathrm{m}\end{array}$ & - & - & - & $\begin{array}{l}\text { Experimental } \\
\text { observations } \\
\text { showed this to be } \\
\text { an important } \\
\text { parameter }\end{array}$ \\
\hline $\begin{array}{l}\text { - Viscosity, } \\
\mathrm{mPa} \text { s }\end{array}$ & 30 (mineral oil) & - & - & $\begin{array}{l}\text { Experimental } \\
\text { observations } \\
\text { showed this to be } \\
\text { an important } \\
\text { parameter }\end{array}$ \\
\hline $\begin{array}{l}\text { - Floating } \\
\text { HL layer } \\
\text { thickness, } \\
\mathrm{mm}\end{array}$ & 10 & Not specified & $1.0-1.6$ & 0.7 \\
\hline \multicolumn{5}{|l|}{$\begin{array}{l}\text { 2. Granular } \\
\text { particles }\end{array}$} \\
\hline $\begin{array}{l}\text { - Density, } \\
\mathrm{kg} / \mathrm{m}^{3}\end{array}$ & $\begin{array}{l}3,800(69 \% \\
\text { zirconia) } \\
6,000(95 \% \\
\text { zirconia) } \\
\end{array}$ & $\begin{array}{l}\text { Considered (not } \\
\text { provided) }\end{array}$ & $\begin{array}{l}\text { 2,650 (quartz } \\
\text { sand) }\end{array}$ & $\begin{array}{l}2,650 \text { (quartz) } \\
2,710 \text { (limestone) } \\
2,350 \text { (clay) }\end{array}$ \\
\hline $\begin{array}{l}\text { - Radius } \\
\text { (average), } \\
\mathrm{mm}\end{array}$ & $\begin{array}{l}0.175(69 \% \\
\text { zirconia) } \\
0.2 \text { (95\% zirconia) }\end{array}$ & $\begin{array}{l}0.0015 \\
\text { (melamine) } \\
0.01 \text { (glass } \\
\text { beads) }\end{array}$ & 0.2 & $\begin{array}{l}0.03 \text { (clay) } \\
0.14 \text { (fine quartz) } \\
0.26 \text { (beach sand) }\end{array}$ \\
\hline
\end{tabular}




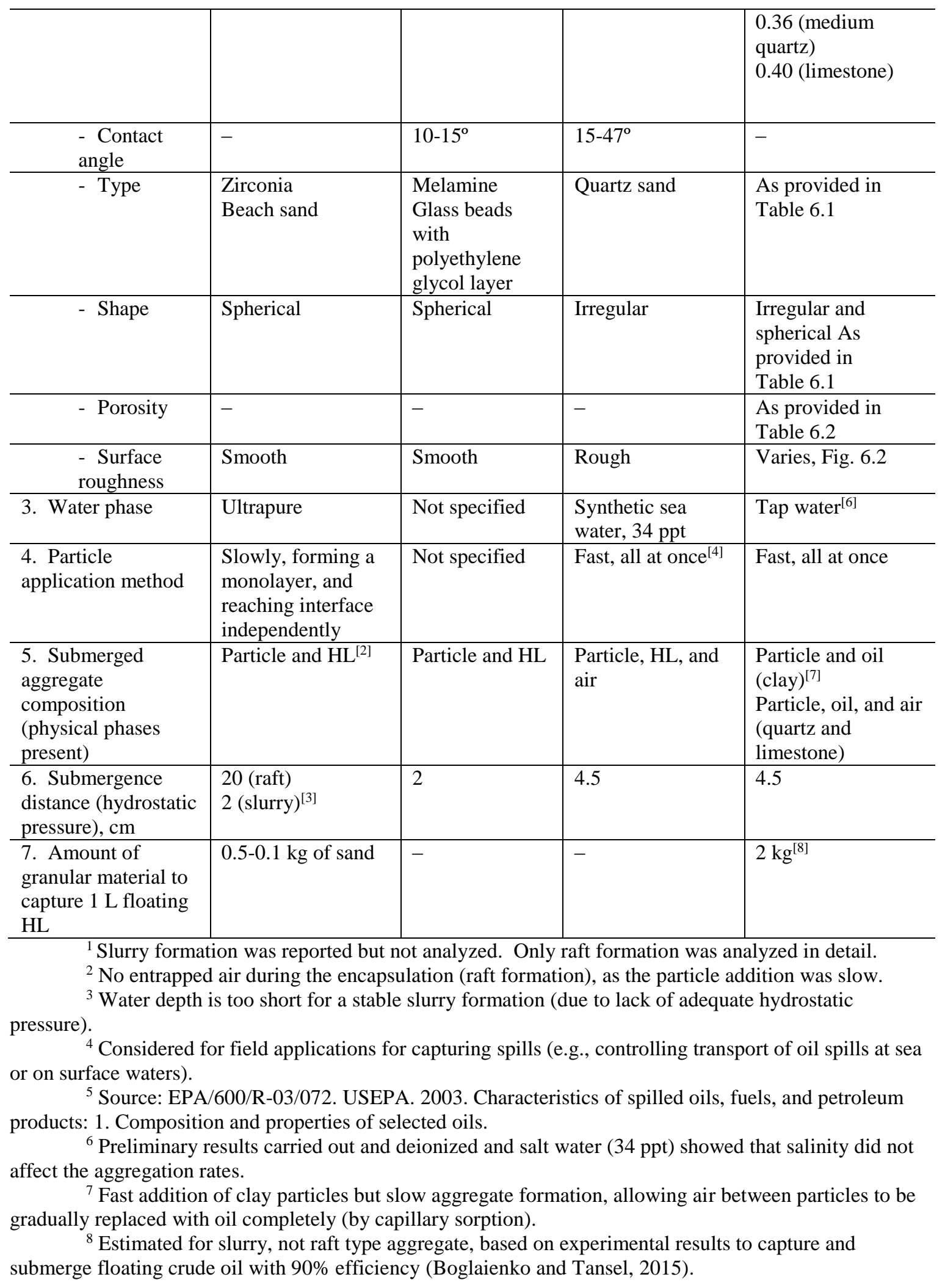


Studies with pure liquids have reported on raft formation or encapsulation with granular particles aligning at the HL-water inteface. The characteristics of the rafts have been studied in terms of particle and HL charecteristics and experimental conditions as summarized in Table 6.3.

However, the characteristics of the rafts (or encapsulation) forming with pure liquids are very different from those forming with crude oil (Fig. 6.5). With SLC, the majority of the granular particles remained impacted inside the HL phase with only a small fraction visible at the HL-water interface with fine quartz, beach sand, and limestone. With clay particles, the aggregates formed very slowly by capillary movement of oil into clay globules. Mathematical equations developed for raft formation scenarios are not applicable due to different physical interactions and physical orientatation of the particles within the aggregate.

Different aggregation types that formed with SLC can be classified as transitional slurry formed by capillary mechanism (i.e., particles are layered outside the hydrophobic phase, as with clay), agglomeration (i.e., particles are outside but partially submerged in the hydrophobic phase, as with medium quartz), and slurry (i.e., some particles are partially outside but majority of the particles are entrapped within the hydrophobic phase, as with fine quartz, beach sand, and limestone).

Clay particles floated on the oil slick allowing oil to be slowly absorbed by capillary forces while floating. Clay particles stayed together mainteining interparticle air space, which was eventually replaced with oil. Once the aggregate was formed, its density increased, causing it to sink. We did not observed air within the submerged aggregate because the process was very slow, allowing air to be gradually replaced by oil. 
The granular materials with larger particle sizes had higher bulk density (Table 6.1) and aggregation occurred instanteneously. However, fine quartz, beach sand and limestone still exhibited some capillary behavior, with partially or fully immersed particles into the hydrophobic globule of crude oil (Fig. 6.1b and 6.1c). The behavior of granular particles with the hydrophobic phase can be grouped as (1) immersion by particle entrapment inside the hydrophobic phase (i.e., slurry) and (2) partial encapsulation by forming a single layer of particles outside the hydrophobic phase (i.e., raft formation), Fig. 6.5. The immersion behavior can be attributed to capillary immersion forces (Kralchevsky and Nagayama, 1994) and may be directed and ordered by electrostatic forces (Danov et al., 2006; Nikolaides et al., 2002). However, this theory is developed for Pickering emulsions (for colloidal particles), where electrostatic forces are relatively important for formation of larger aggregates. Conversely, the systems with granular particles also have significant gravitational forces, which effect the penetration of particles into the hydrophobic phase. Further investigations are needed to characterize the behavior of granular particles in relation to encapsulation of the hydrophobic phase vs immersion and entrapment within the hydrophic phase while densifying and immobilizing the floating phase in aqueous medium.

Quartz sand with medium particle size did not form slurry type aggregation, but rather an agglomerate with smaller wetting angles, hence, they were mostly positioned outside the aggregate. SEM images revealed that the surface of the medium quartz sand particles is smoother than the limestone particles (with similar particle size) (Fig. 6.2a and 6.2d); specific pore surface measured as the relation of the surface pores to total surface were 0.12 and 0.18 , respectively (Table 6.2). As it can be seen from Figure $6.2 \mathrm{~d}$, the pores 
within the limestone particles penetrate deeper into the body of the material (sponge-like), which may explain better wetting properties and higher aggregation efficiency with oil in comparison to that for quartz sand with similar grain size. The specific pore surface areas of quartz sand with fine and medium particle sizes are similar, 0.11 and 0.12 respectively. Despite the average size of the surface pores of the fine quartz particles is more than twice the average pore size of the medium quartz particles (Fig. 6.2a and 6.2b), the aggregation of medium quartz sand with oil has higher rank and less floating oil remaining (Table 6.2). Beach sand is considered as a mixture of quartz and limestone particles (Table 6.1, ESM data on composition). The performance of beach sand was the highest among the granular materials studied (Fig. 6.1a) but it was noticed that aggregation rates may vary depending on the method the granular material is applied. Specific pore surface area and rank of aggregation of beach sand were the highest ( 0.31 and rank 1$)$ amongst the materials studied. Finally, coarse limestone showed very small aggregation with crude oil, due to its relatively larger particle size, which may have caused the particles to move through the floating layer rapidly, preventing them to aggregate and capture oil effectively (Fig. 6.1a).

The observations and experimental data showed that particle size, and surface porosity (or roughness) of granular particles affect their aggregation potential with crude oil, however, the size of the surface pores appear to be not a signifiant factor (Fig. 6.3). It is in agreement with the findings of the authors Halsey and Levine (1998), who concluded that surface roughness of such material as sand controlls the strengths of capillary bridges. Also, there appears to be an optimum particle size range $(<2.00 \mathrm{~mm}$ of a mesh opening $)$ that allows effective aggregation while the particles pass through the floating oil layer. 
Additional experiments were performed with clay and decane (as a representative of the alkane group, the main constituent of crude oil) to compare the effect of polar functional groups that are present in crude oil with a nonpolar hydrophobic substance (decane). As shown in Fig. 6.4, the presence of the polar compounds (asphaltenes) in crude oil effects the interaction of granular particles with crude oil significantly. Asphaltenes are known to induce wettability of crude oil on mineral surfaces (Yan et al., 1997; Zhen et al., 2001), and as demonstrated by the experiments, the aggregation characteristics of clay with crude oil versus clay and decane occur by significantly different mechanisms.

\section{Conclusions}

In this study, we experimentally evaluated the performance of granular materials for capturing floating crude oil. Sand (two particle sizes), limestone (two particle sizes), beach sand and clay were used as the granular materials. Densification and submergence of the floating oil occurred by partially or fully entrapment of particles within the oil phase. This is a different mechanisms than the encapsulation (or raft formation) reported with pure oils (Abkarian et al., 2013) and pure hydrophobic liquids (i.e., silicone oil, alkanes). The observed aggregates formed with initially floating crude oil can be classified as transitional slurry formed by capillary mechanism (clay), agglomeration (medium quartz), and slurry (fine quartz, beach sand, and limestone). High surface porosity of the granular particles resulted in higher capture efficiencies of the floating oils per unit weight of granular material applied. However, there is an optimum particle size range (smaller than $2.00 \mathrm{~mm}$ opening of 10 mesh) that allows effective aggregation as the particles pass through the floating oil layer. 
Surface pore size did not have a significant effect on oil capture efficiency. The experimental results provide the insight to investigate the capillary and electrostatic forces acting on granular particles in oil aggregates. These systems with granular particles are different from the well-studied emulsions and colloidal suspensions.

\section{Acknowledgments}

Partial funding for this research has been provided by Gulf of Mexico Research Initiative (GoMRI) through funding to Consortium for the Molecular Engineering of Dispersant Systems (CMEDS).

\section{References}

Kvenvolden, K.A., and Cooper, C.K. 2003. Natural seepage of crude oil into the marine environment. Geo-Marine Letters, 23: 140-146.

Input of Oil to the Sea. Oil in the Sea III: Inputs, Fates, and Effects [Online]. National Academy of Sciences, National Academies Press, Washington, DC, 2003; pp 6588. http://www.nap.edu/read/10388/chapter/4\#67 (accessed Oct 8, 2015).

Tansel, B. 2014. Propagation of impacts after oil spills at sea: Categorization and quantification of local vs regional and immediate vs delayed impacts. International Journal of Disaster Risk Reduction, 7: 1-8.

Tansel, B., Lee, M., and Tansel, D.Z. 2013. Comparison of fate profiles of PAHs in soil, sediments and mangrove leaves by QSAR and QSPR. Marine Pollution Bulletin, 73: $258-262$.

Tansel, B., Lee, M., Berbakov, J., Tansel, D.Z., and Koklonis, U. 2014. Dispersion of Louisiana crude oil in salt water environment by Corexit 9500A in the presence of natural coastal materials. Estuarine, Coastal and Shelf Science, 143: 58-64.

Making Decisions about Dispersant Use. Oil Spill Dispersants: Efficacy and Effects [Online]. National Academy of Sciences, National Academies Press. Washington, DC, 2005; pp 21-50. http://www.nap.edu/read/11283/chapter/4 (accessed Oct 8, 2015). 
Dispersant Use Approvals in the United States. Factsheet No. 5. Oil Spill Prevention. http://www.oilspillprevention.org/ /media/oil-spill-prevention/spillprevention/rand-d/dispersants/5-dispersant-use-approvals-in-the-united.pdf (accessed Oct 8, 2015).

Payne, J. R., Kirstein, B. E., Clayton, J. R., Clary, C., Redding, R., McNabb, D., and Farmer, G. Integration of suspended particulate matter and oil transportation study; Final Report: Science Application International Corp., San Diego, CA, September 1984-September 1987. No. PB-88-116421/XAB; SAIC-87/ETG-2.

Bandara, U. C., Yapa, P. D., and Xie, H. 2011. Fate and transport of oil in sediment laden marine waters. Journal of Hydro-environmental Resources, 5: 145-156.

Gong, Y., Zhao, X., Cai, Z., O’Reilly, S. E., Hao, X., and Zhao, D. 2014. A review of oil, dispersed oil and sediment interactions in the aquatic environment: influence on the fate, transport and remediation of oil. Marine Pollution Bulletin, 79: 16-33.

Lee, K. 2002. Oil-particle interactions in aquatic environments: influence on the transport, fate, effect and remediation of oil spills. Spill Science \& Technology Bulletin, 8: 38.

Owens, E. H., and Lee, K. 2003. Interaction of oil and mineral fines on shoreline: review and assessment. Marine Pollution Bulletin, 47: 397-405.

Sterling, M.C., Bonner, J.S., Page, C.A., Fuller, C.B., Ernest, A.N., and Autenrieth, R.L. 2004. Modeling crude oil droplet-sediment aggregation in nearshore waters. Environmental Science \& Technology, 38: 4627-4637.

Bragg, J. R., and Owens, E. H. 1995. Shoreline cleansing by interactions between oil and fine mineral particles. In Proc. Int. Oil Spill Conf., Long Beach, CA, American Petroleum Institute, Washington, DC, Publication No. 4620, Feb 22-Mar 2, 1995; pp 219-227.

Bragg, J.R., and Yang, S. H. 1996. Method for forming mineral solids-oil floccules. U.S. Patent US5490940 A. Feb 13, 1996.

Muschenheim, D.K., and Lee, K. 2002. Removal of oil from the sea surface through particulate interactions: review and prospectus. Spill Science \& Technology Bulletin, 8: 9-18.

Boglaienko, D., and Tansel, B. 2015. Instantaneous stabilization of floating oils by surface application of natural granular materials (beach sand and limestone). Marine Pollution Bulletin, 91: 107-112. 
Boglaienko, D., Tansel, B., and Sukop, M.C. 2016. Granular encapsulation of light hydrophobic liquids (LHL) in LHL-salt water systems: Particle induced densification with quartz sand. Chemosphere, 144: 1358-1364.

Abkarian, M., Protiere, S., Aristoff, J.M., and Stone, H.A. 2013. Gravity-induced encapsulation of liquids by destabilization of granular rafts. Nature Communications, 4 (1895).

Kralchevsky, P.A., and Nagayama, K. 1994. Capillary forces between colloidal particles. Langmuir, 10: 23-36.

Danov, K.D., Kralchevsky, P.A., Ananthapadmanabhan, K.P., and Lips, A. 2006. Particleinterface interaction across a nonpolar medium in relation to the production of particle-stabilized emulsions. Langmuir, 22: 106-115.

Nikolaides, M.G., Bausch, A.R., Hsu, M.F., Dinsmore, A.D., Brenner, M.P., Gay, C., and Weitz, D.A. 2002. Electric-field-induced capillary attraction between like-charged particles at liquid interfaces. Nature, 420 (6913): 299-301.

Halsey, T.C., and Levine, A.J. 1998. How sandcastle fall. Physical Review Letters, 80: 3141-3144.

Yan, J., Plancher, H., and Morrow, N.R. 1997. Wettability changes induced by adsorption of asphaltenes. SPE Production \& Facilities, 12: 259-266.

Zheng, J., Behrens, S.H., Borkovec, M., and Powers, S.E. 2001. Predicting the wettability of quartz surfaces exposed to dense nonaqueous phase liquids. Environmental Science \& Technology, 35: 2207-2213.

Tavacoli, J.W., Katgert, G., Kim, E.G., Cates, M.E., and Clegg, P.S. 2012. Size limit for particle-stabilized emulsion droplets under gravity. Physical Review Letters, 108: 268306 (5). 


\title{
CHAPTER VII
}

\section{SUBMERGENCE PATTERNS OF FLOATING CRUDE OIL BY GRANULAR PARTICLES}

Boglaienko, D., and Tansel, B. 2017. Chemical Engineering Journal, 314: 548-553.

DOI: 10.1016/j.cej.2016.12.012

\begin{abstract}
Granular particles added to floating oils can transform the floating layer to submerged state. Here we show that there is a critical thickness at which the floating oil can hold large amounts of particles by forming an elastic oil-particle bowl while keeping some particles in completely dry state. Furthermore, we observed that at high particle application rates, a vertical channel forms in water through which floating oil submerges rapidly. Transformation of floating liquid to high strength elastic state has important implications for particle induced reinforcement at liquid-liquid interfaces; while formation of liquid-in-liquid channels by granular particles enables pipeless and rapid transfer of floating liquids.
\end{abstract}

\section{Introduction}

Understanding the behavior of light hydrophobic liquids in water and their interaction with granular particles is needed for controlling the fate of oil spills in the environment, as well as for development of treatment processes and oil spills mitigation strategies (Abkarian et al., 2013; Boglaienko and Tansel, 2015). 
The aggregation of light hydrophobic liquids with granular materials is similar to liquid marble formation, however, unlike liquid marbles, which are liquid droplets encapsulated by hydrophobic particles exposed to air (Walker et al., 2013; Aussillous and Quere, 2001; Aussillous and Quere, 2006), the particle-oil aggregates are hydrophobic liquids encapsulated by granular solids in water (Boglaienko and Tansel, 2016a; Boglaienko and Tansel, 2016b; Tavacoli et al., 2012).

Encapsulation or, in our case, aggregation of hydrophobic liquids with granular particles depends on factors that can be divided into two major categories: physical and chemical properties, and application mode. Physical properties of hydrophobic liquids, such as density, viscosity, and surface and interfacial tension affect aggregation rates with granular materials (Boglaienko and Tansel, 2016a; Bormashenko et al., 2013). Particles size, wettability, and surface morphology are related to aggregation efficiency (Boglaienko and Tansel, 2016b; Nguyen et al., 2010), and capillary cohesion (Domenech and Velankar, 2015). Chemical composition of both liquids and granular materials influences transport and positioning of particles at the hydrophobic liquid and water interface (Boglaienko and Tansel, 2017). Application mode is a less studied category of parameters, as it depends on the experimental design and test conditions. Particle to liquid ratio (Boglaienko and Tansel, 2015), or volume fraction (Tavacoli et al., 2012), has been considered as the most important application factor for the particle-oil formation. However, application rate has not been evaluated for this type of particle and oil interactions. Application rate (mass of particles per unit of time) was controlled in this study as the main parameter. Different submergence and aggregation patterns of oil occurred with granular particles (i.e., vertical liquid bridging, or roping state, and capillary bridging for particle retention, or bowl state). Thus, 
the main objective was to investigate the interactions of granular particles, which were applied at different flow rates, with floating oil layer of different thicknesses.

Regarding the application mode of particles, different flow regimes have been studied which affect mobility characteristics of the granular particles (Forterre and Pouliquen, 2008; Coussot, 2005; Coussot and Ancey, 1999; Ovarlez et al., 2010). These flow regimes have been classified as solid, liquid, and gaseous types depending on interaction of particles (Forterre and Pouliquen, 2008). Behavior of particles is very different in each flow regime and in the transition states (i.e., jamming transition from liquid to solid flow (Ovarlez et al., 2010). However, the interaction of the granular particle flow with immiscible liquids involving a hydrophobic liquid-water interface and different submergence patterns have not been reported in the literature.

The investigation of submergence patterns of floating oil by granular particles opens up the possibilities for transformation of light hydrophobic liquids to different states. These transformations can be used to explore novel technologies for capturing and stabilizing light hydrophobic liquids in a controlled manner. Further investigations of the particle-oil interactions can lead to development of efficient treatment methods which can be as effective as the conventional methods employed for oil and grease removal (Pintor et al., 2016). Additionally, the submergence patterns and characteristics of the particle-oil aggregates provide insight into the mechanisms for breaking the floating oils under the influence of surface tension and gravity forces. 


\section{Materials and Methods}

Experiments were conducted with South Louisiana crude oil (MC 252), obtained from the BP America Production Company (Houston, TX), and quartz sand with two different particle sizes. Pure quartz sand with fine particle size (40-100 mesh that corresponds to particle size range of $0.42-0.15 \mathrm{~mm}$; average $0.28 \mathrm{~mm}$ ) was obtained from Acros Organics; pure quartz sand with medium particle size (20-30 mesh with particle size range of 0.85-0.60 mm; average $0.72 \mathrm{~mm}$ ) was obtained from Spectrum Chemical MFG.

Submergence experiments were performed in a $50 \mathrm{~mL}$ beaker filled with tap water and placed on an electronic balance to monitor the addition rate of the particles. Quartz sand was applied to the floating South Louisiana crude oil layer through a funnel equipped with a stopper. The application rate of sand was controlled by using funnels with different size openings. The distance from the funnel opening to the floating oil surface was maintained the same $(8 \mathrm{~cm})$. The experiments were carried out at the room temperature $\left(22{ }^{\circ} \mathrm{C}\right)$.

Sand particles, reaching the floating layer, initiated instantaneous aggregation followed by retention and/or submergence of the particle-oil aggregates. Transition time for the submergence patterns varied, but for the majority of the experiments it occurred within several seconds. The granular particles passed through the floating oil without aggregation in less than one second. Vertical transfer of the floating oil by roping also occurred within one second. The time needed for the transition of oil from the floating to the submerged state as a small aggregate was $1-2 \mathrm{sec}$, and, as a large aggregate with a significant amount of sand retained (bowl formation), was $10 \mathrm{sec}$ on average. 
The thickness $(h, \mathrm{~mm})$ of crude oil layer was varied during the experiments. The amount (volume, $V, \mathrm{~mL})$ of oil added was estimated by mass $(m, \mathrm{~g})$ and density $\left(\rho, \mathrm{g} / \mathrm{cm}^{3}\right)$ of the oil and the radius $(r, \mathrm{~mm})$ of the oil slick $\left(h=V / \pi r^{2}\right.$, or $\left.h=m / \rho \pi r^{2}\right)$.

Impact force of a particle was calculated as $F=m_{p} g h_{l} / d$, knowing mass of a particle $\left(m_{p}, \mathrm{~kg}\right)$; acceleration due gravity $\left(\mathrm{g}, \mathrm{m} / \mathrm{s}^{2}\right)$; distance from a funnel opening to liquid surface $(h,, \mathrm{~m})$; and travel distance of a particle in oil after impact $(d, \mathrm{~m})$.

\section{Results and Discussion}

The experiments conducted with crude oil and granular sand particles showed that there were six distinct submergence patterns of the particle-oil aggregates depending on the floating oil thickness, particle size, and particle addition rate (Table 7.1). These patterns were described as pass through (particles coated with thin oil film and submerged discretely), small aggregates (particles aggregated within submerging liquid oil drop), small and large aggregates, bowl formation (particles retained in the floating state, while forming a strong elastic paste), aggregates followed by bowl formation, and liquid roping (formation of a liquid pipe through which oil and particles submerged rapidly).

When the floating oil layer was thin $(0.03 \mathrm{~mm})$ and the ratio of particle size to oil layer thickness was high (up to particle size / oil layer $=25$ and higher), particles, regardless of their size and application rate, were able to pass through the oil rapidly by the gravitational forces (Fig. 7.1a, region I). As the particles passed through the thin oil layer, they became hydrophobic. The surface of particles was observed to be covered by thin oil film. Because the thickness of oil film did not allow capillary bridging between particles, 
they submerged discretely. We described this thin oil film coating on the particles as immobile oil shell (Fig. 7.1b, scheme a).

Increasing floating oil layer thickness led to formation of both small and large aggregates (Fig. 7.1a, regions IV and V respectively). As the particle size to oil thickness ratio decreased, or the floating oil layer thickness increased $(0.14-1.21 \mathrm{~mm})$, the particleoil aggregates became larger. The thicker liquid oil shell around the particles functioned as the joining medium for particle-oil aggregates (Fig. 7.1b, scheme c). The relatively high application rate $(0.87 \mathrm{~g} / \mathrm{s})$ of the particles at low ratios of particle size to oil thickness (around 1:1) resulted in higher amounts of oil to be captured in the aggregates that submerged through the water phase. The submerging aggregates contained oil in liquid state with the granular particles positioned at the oil-water interface and within the hydrophobic body of the aggregate, - slurry type (Boglaienko and Tansel, 2016b).

Further increase of the particle addition rate $(1.58 \mathrm{~g} / \mathrm{s}$ for the quartz sand with average particle size $0.28 \mathrm{~mm}$ ) resulted in a phenomenon that we call roping, or liquid pipe (Fig. 7.1a, region VI). Roping state occurred during the liquid type of granular flow regime (Forterre and Pouliquen, 2008). Regardless of the thickness of the floating oil layer, the impact force $(0.085 \mathrm{mN}$ per particle) caused formation of a tail behind the submerging particle-oil aggregates as the particles settled at a faster rate than the oil surrounding them. Vertical merging of liquid tails formed a liquid channel in water through which the floating oil submerged rapidly (Fig. 7.1b, scheme b). Formation the oil channel resulted in submergence of essentially the entire floating oil layer with no visible residual oil remaining in the floating state. 
Another observed phenomenon that has not been reported in the literature was the formation of so-called bowl state, at which the floating particle-oil aggregate was able to hold particles over 10 times the weight of the oil slick itself. At the bowl state, the granular particles formed a strong paste-like slurry which retained practically half of the particles in a completely dry state (without coming in contact with oil and water) while still floating. At this state, the oil-water interface exhibited high stretching ability with the increasing weight of the particles. In the series of experiments conducted (using both types of quartz sand with average particle sizes 0.28 and $0.72 \mathrm{~mm}$ ), the thickness of the oil layer at which the bowl formation occurred was between 0.06 to $0.17 \mathrm{~mm}$ for fine sand and between 0.06 to $0.15 \mathrm{~mm}$ for medium sand $(0.10 \mathrm{~mm}$ on average $)$, as shown in Fig. 7.2a,b. It is important to note that the addition rate of the granular particles should be small enough $(0.20-0.32$ g/s; gaseous type granular flow regime (Forterre and Pouliquen, 2008) not to break the floating particle-oil aggregate that is carrying the particles (Fig. 7.1a, region II). In the bowl state, the liquid oil shell saturates with particles, decreasing radius of the shell while immobilizing the particles as it transitions to the paste-like state. At this state the particles are held together tightly by capillary bridging (Fig. 7.1b, scheme d). Bowl formation resulted in capture and removal of almost all the floating oil when it eventually submerged with the added weight of the particles.

With the increasing rate of granular particle addition $(0.87 \mathrm{~g} / \mathrm{s})$ particle-oil aggregates submerged without forming a bowl (transition of the region II to region IV). When the amount of floating oil increased $(0.23-0.56 \mathrm{~mm})$, or the ratio of particle size to oil layer thickness was reduced, particle-oil aggregates formed and submerged until the oil 
layer reached the critical thickness for the bowl formation (transition of the region II to region III that has both aggregates and bowl formation states), Fig. 7.1a.

Figure 7.2 illustrates the observations from the four sets of experiments that resulted in the phenomena described above. Quartz sand with particle sizes 0.28 and $0.72 \mathrm{~mm}$ resulted in a floating granular reinforced state which formed the bowl state due to increased elasticity of the particle-oil aggregate at sand addition rates 0.20 and $0.32 \mathrm{~g} / \mathrm{s}$, respectively. When the oil thickness was above the critical thickness for the bowl state (over 0.15-0.17 $\mathrm{mm}$ ), particle-oil aggregates formed and submerged initially (Fig. 7.2a,b). Interestingly, the thicker the oil slick was at the start of the sand addition, the smaller the first particleoil aggregate formed and submerged from the floating phase, followed by formation of larger aggregates. The largest aggregate was formed just before transitioning of the floating oil to the bowl state. We relate this formation of the larger submerging aggregate to the increase in cohesive interactions due to proximity of the particles to each other. As the thickness of the remaining oil layer was reduced, the particle-particle interactions became stronger because of the bridging phenomenon. Overall, at the high addition rates with both particle sizes ( 1.58 and $0.87 \mathrm{~g} / \mathrm{s}$ ), simpler submergence patterns were observed (i.e., roping and formation of aggregates only), Fig. 7.2c,d.

We find bowl formation as a unique state of transformation of a hydrophobic liquid to a paste-like state that is able to retain its shape. Accumulation of particles starts in the middle of the impact area, growing to the sides of the oil layer, adsorbing floating oil while holding additional layers of particles. The bowl can accumulate a layer of sand over $5 \mathrm{~mm}$ thick (Fig. 7.3a-c). This thickness is above the capillary length for South Louisiana crude oil and water $\left(a=(\gamma / \rho g)^{1 / 2}, a=1.76 \mathrm{~mm}\right.$ for oil and $2.7 \mathrm{~mm}$ for water) and the sand 
particles accumulating on the top layers remain dry. Once the bowl stretches enough by the increasing weight of the granular particles, the edges become weaker. As the bowl continues to stretch, it breaks from the side, allowing water to enter, causing the bowl to break (Fig. 7.3a,b,c). An analogy can be drawn with the observations from wicking experiments (Raux et al., 2013), where glass beads (with $0.256 \mathrm{~mm}$ radius) were deposited as a floating layer at the interface between ethanol bath and air. The significant difference is that, once the pile of beads was impregnated by ethanol, a single bead was detached and submerged. In our experiments, the particle-oil bowl acted as a unified body holding all the particles while the hydrophobic-hydrophilic interface became reinforced. Other hydrophobic liquids were also tested for bowl formation and exhibited similar unified state. Fig. 7.3d,e shows decane (dyed with Sudan IV for ease of observation). Even after the bowl breaks and submerges, it continues to retain its shape.

Another notable hydrophobic liquid and particle aggregate formation, which was referred as raft (Abkarian et al., 2013), was also observed (Fig. 7.3e). This type of aggregation was reported by Abkarian et al. (2013), and it represented a specific state of interaction and submergence pattern of hydrophobic liquids with granular particles. 
Table 7.1. Submergence patterns of floating crude oil with granular particles. Identified regions refer to Fig. 7.1a.

\begin{tabular}{|c|c|c|}
\hline Submergence pattern & $\begin{array}{l}\text { Dominant effects and } \\
\text { forces }\end{array}$ & \\
\hline $\begin{array}{l}\text { Pass through } \\
\text { (Region I) }\end{array}$ & Gravitational & 5 \\
\hline $\begin{array}{l}\text { Small aggregates } \\
\text { (Region IV) }\end{array}$ & $\begin{array}{l}\text { Gravitational } \\
\text { Cohesion } \\
\text { Capillary bridging }\end{array}$ & \\
\hline $\begin{array}{l}\text { Small and large } \\
\text { aggregates } \\
\text { (Regions IV and V) }\end{array}$ & $\begin{array}{l}\text { Gravitational } \\
\text { Cohesion } \\
\text { Capillary bridging } \\
\text { Viscous stretching and } \\
\text { pinch off }\end{array}$ & \\
\hline $\begin{array}{l}\text { Bowl formation } \\
\text { (Region II) }\end{array}$ & $\begin{array}{l}\text { Gravitational } \\
\text { Cohesion } \\
\text { Capillary bridging } \\
\text { Viscous stretching (no } \\
\text { pinch off) } \\
\text { Elastic/Plastic } \\
\text { stretching }\end{array}$ & \\
\hline $\begin{array}{l}\text { Aggregates followed } \\
\text { by bowl formation } \\
\text { (Region III) }\end{array}$ & $\begin{array}{l}\text { Gravitational } \\
\text { Cohesion } \\
\text { Capillary bridging } \\
\text { Viscous stretching and } \\
\text { pinch off } \\
\text { Followed by: } \\
\text { Viscous stretching (no } \\
\text { pinch off) } \\
\text { Elastic/Plastic } \\
\text { stretching }\end{array}$ & \\
\hline $\begin{array}{l}\text { Roping } \\
\text { (Region VI) }\end{array}$ & $\begin{array}{l}\text { Gravitational } \\
\text { Cohesion } \\
\text { Inertial effects and } \\
\text { jetting } \\
\text { Viscous stretching (no } \\
\text { pinch off) }\end{array}$ & \\
\hline
\end{tabular}


a

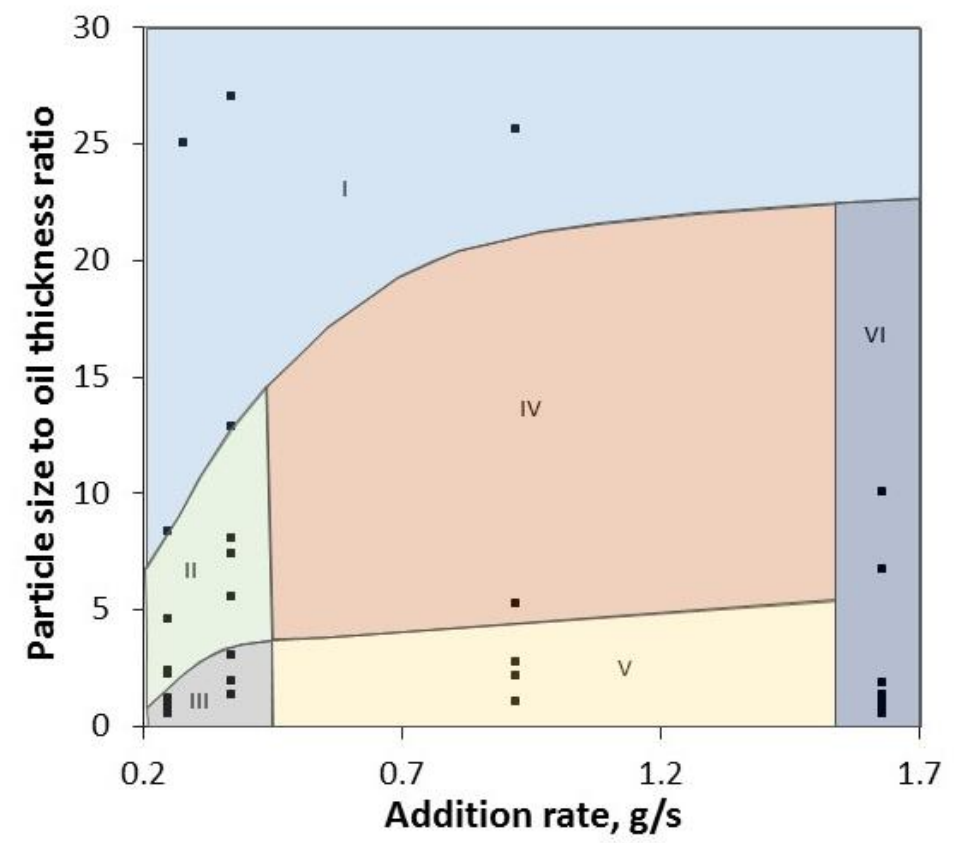

b

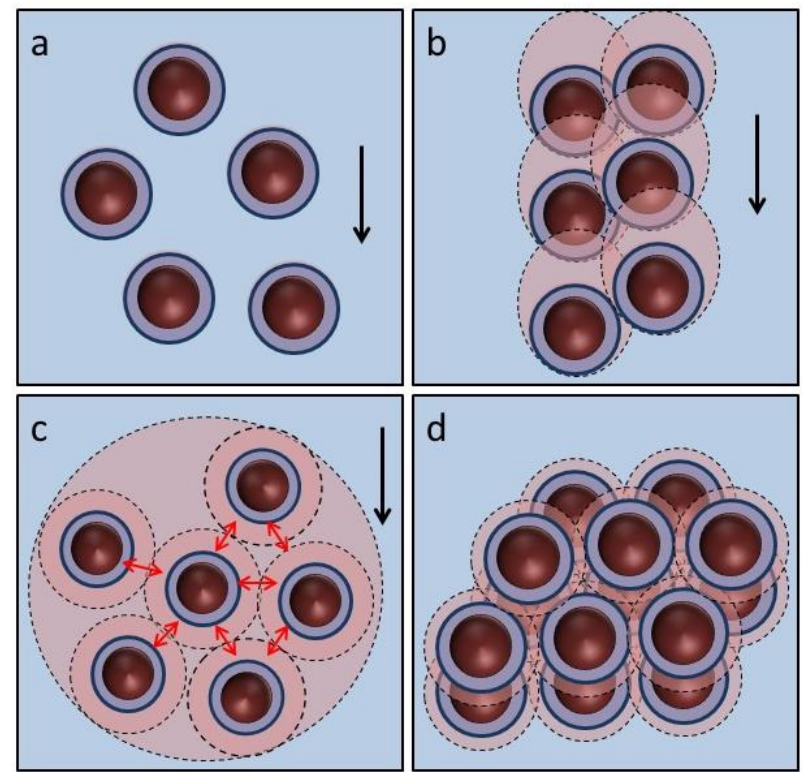

Figure 7.1. Submergence patterns and granular interactions: a. Conceptual submergence pattern diagram for South Louisiana crude oil and quartz sand (average particle sizes 0.28 and $0.72 \mathrm{~mm}$ ), regions are: I pass through, II bowl formation, III aggregate following bowl formation, IV small aggregates, V large aggregates, and VI roping state. Division lines between different submergence states are approximate. b. Behavior of granular particles in different states and interaction with oil: (a) immobile oil shell during pass through state (very thin oil film on the particles), (b) elongation of mobile oil shell due to viscous interactions during submergence in roping state, (c) mobile oil shell interactions during large aggregate formation, and (d) immobilization of mobile shell during bowl formation. 
a

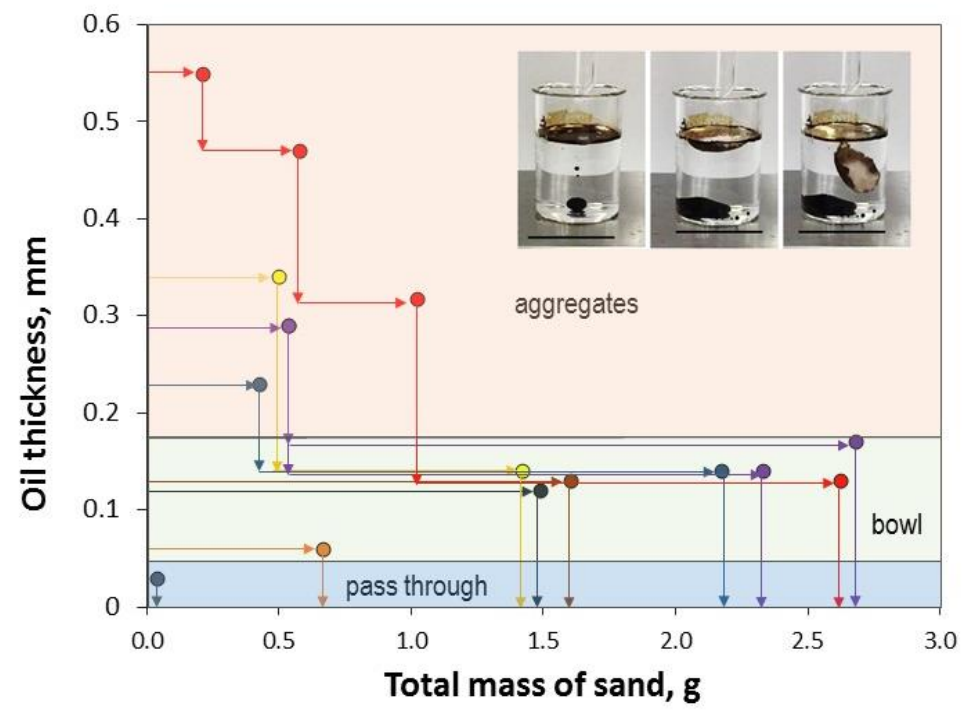

b

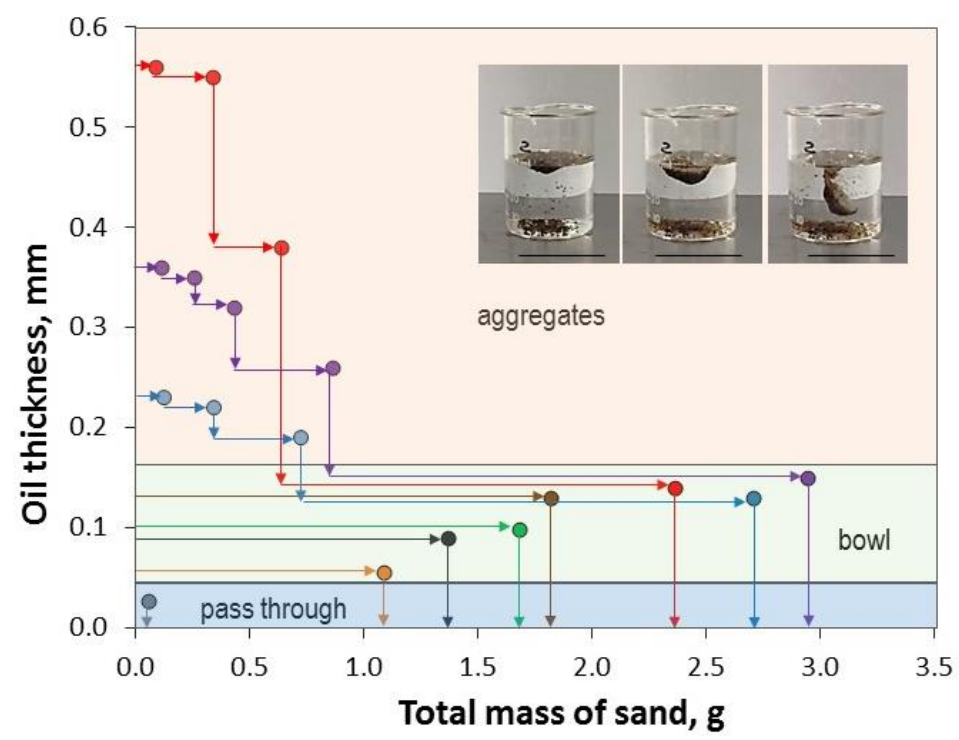




\section{C}
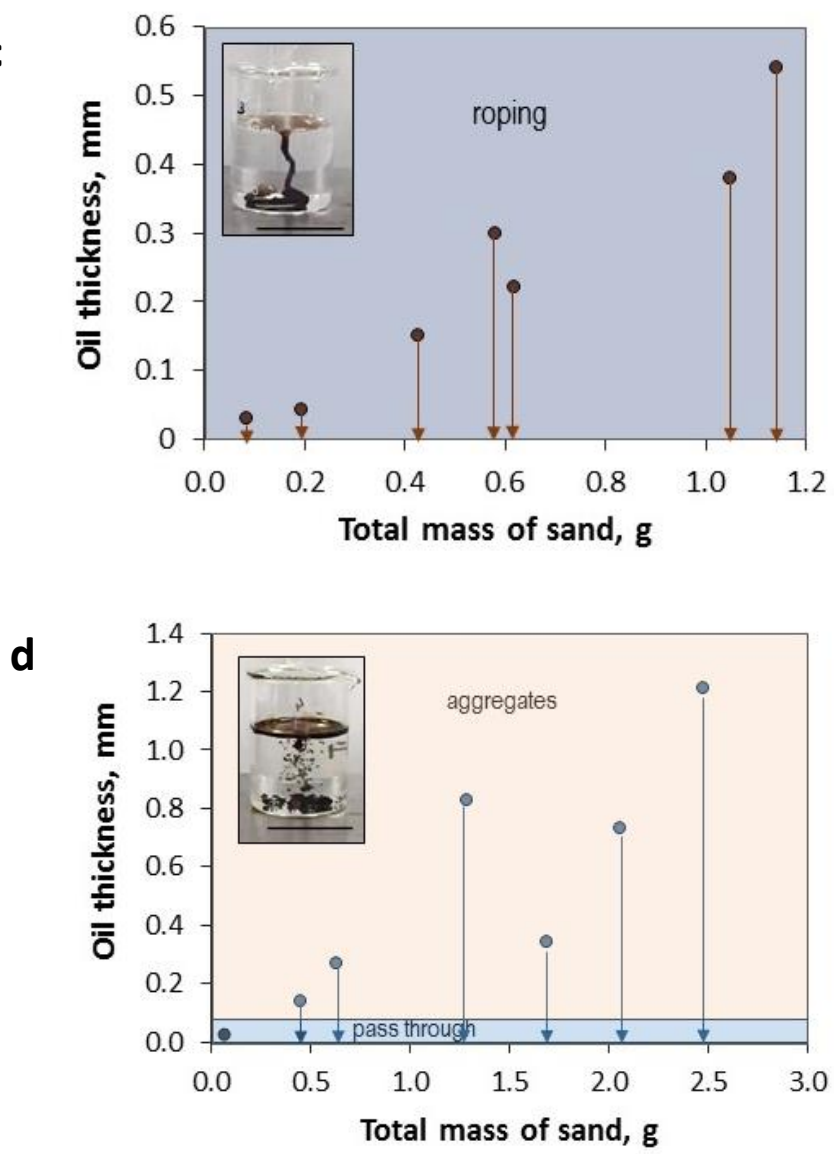

Figure 7.2. Submergence patterns of granular particles: a. Quartz particles with $0.28 \mathrm{~mm}$ average size at $0.20 \mathrm{~g} / \mathrm{s}$ application rate; b. Quartz particles with $0.72 \mathrm{~mm}$ average size at $0.32 \mathrm{~g} / \mathrm{s}$ application rate; c. Quartz particles with $0.28 \mathrm{~mm}$ average size at $1.58 \mathrm{~g} / \mathrm{s}$ application rate; and d. Quartz particles with $0.72 \mathrm{~mm}$ average size at $0.87 \mathrm{~g} / \mathrm{s}$ application rate. The same color represents the submergence states during each test condition (for example, aggregate formation-bowl-pass through; or bowl-pass through; or pass through only, depending on the oil thickness at the starting point). Scale bar on photos: $4 \mathrm{~cm}$. Colors identifying each region are the same as defined in Fig. 7.1a. 

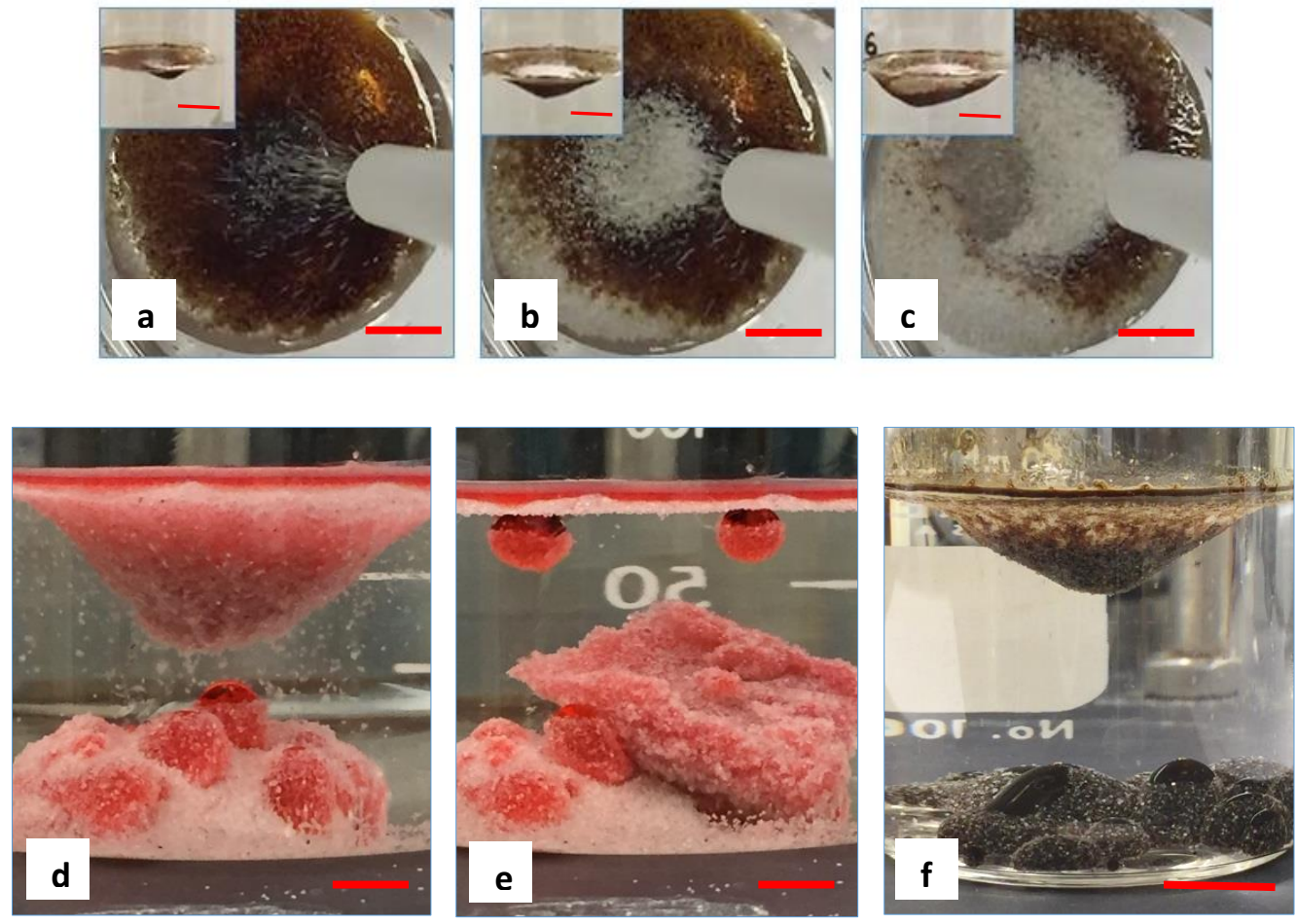

Figure 7.3. Occurrence of bowl state: a-c. Top and side views of bowl formation; d, e. Bowl formation with decane (dyed with Sudan red) and quartz particles $(0.28 \mathrm{~mm}) ;(\mathbf{e})$ submerged bowl (paste-like aggregate); near spherical globules below surface are the raft type of hydrophobic liquid-particle aggregates. f. Strength and elasticity of the floating aggregate that formed at the critical oil thickness which resulted in bowl formation (floating) and large aggregates that submerged when the oil layer was above the critical thickness (submerged) prior to bowl formation. Scale bar: $1 \mathrm{~cm}$.

\section{Conclusion}

In conclusion, our observations of the bowl and roping (liquid pipe) states provide possibilities for using granular materials to capture and control floating oils by phase transformation (i.e., as floating paste or submerged state). The floating bowl state can have applications for handling and processing immiscible liquids. The high strength of the particle-oil aggregate at the bowl state provides insight on the conditions that lead to loss of particle transfer through liquid-liquid interfaces. Occurrence of the critical state at which the particle transfer through the floating layer is prevented can be used for granular 
enforcement of liquid-liquid interfaces. Another important observation is the formation of the liquid pipe which provides transfer of the floating oil to the submerged state rapidly. This allows the concept of using granular particles for pipeless transfer in immiscible liquid systems. From a general application point of view, our results demonstrate use of granular particles for phase transformation of light hydrophobic liquids.

\section{Acknowledgments}

The authors acknowledge funding from Florida International University, Dissertation Year Fellowship.

References

Abkarian, M., Protiere, S., Aristoff, J.M., and Stone, H.A. 2013. Gravity-induced encapsulation of liquids by destabilization of granular rafts. Nature Communications, 4 (1895).

Boglaienko, D., and Tansel, B. 2015. Instantaneous stabilization of floating oils by surface application of natural granular materials (beach sand and limestone). Marine Pollution Bulletin, 91: 107-112.

Walker, G.M., McEleney, P., Al-Muhtaseb, A.H., and Bell, S.E.J. 2013. Liquid marble granulation using super-hydrophobic powders. Chemical Engineering Journal, 228: 984-992.

Aussillous, P., and Quere, D. 2001. Liquid marbles. Nature, 411: 924-927.

Aussillous, P., and Quere, D. 2006. Properties of liquid marbles. Proceedings of the Royal Society A, 462: 973-999.

Boglaienko, D., and Tansel, B. 2016a. Encapsulation of light hydrophobic liquids with fine quartz sand: property based characterization and stability in aqueous media with different salinities. Chemical Engineering Science, 145: 90-96. 
Boglaienko, D., and Tansel, B. 2016b. Gravity induced densification of floating crude oil by granular materials: effect of particle size and surface morphology. Science of The Total Environment, 556: 146-153.

Tavacoli, J.W., Katgert, G., Kim, E.G., Cates, M.E., and Clegg, P.S. 2012. Size limit for particle-stabilized emulsion droplets under gravity. Physical Review Letters, 108: 268306.

Bormashenko, E., Musin, A., Whyman, G., Barkay, Z., Starostin, A., Valtsifer, V., and Strelnikov, V. 2013. Revisiting the surface tension of liquid marbles: Measurement of the effective surface tension of liquid marbles with the pendant marble method. Colloid Surface A, 425: 15-23.

Nguyen, T.H., Hapgood, K., and Shen, W. 2010. Observation of the liquid marble morphology using confocal microscopy. Chemical Engineering Journal, 162: 396405 .

Domenech, T., and Velankar, S.S. 2015. On the rheology of pendular gels and morphological development in paste-like ternary systems based on capillary attraction. Soft Matter, 11: 1500-1516.

Boglaienko, D., and Tansel, B. 2017. Preferential positioning and phase exposure of granular particles at hydrophobic liquid-water interface. Journal of Cleaner Production, 142: 2629-2636.

Forterre, Y., and Pouliquen, O. 2008. Flows of dense granular media. Annual Review of Fluid Mechanics, 40: 1-24.

Coussot, P. 2005. Rheometry of Pastes, Suspensions, and Granular Materials. Wiley.

Coussot, P., and Ancey, C. 1999. Rheophysical classification of concentrated suspensions and granular pastes. Physical Review E, 59 (4445).

Ovarlez, G., Barral, Q., and Coussot, P. 2010. Three-dimensional jamming and flows of soft glassy materials. Nature Materials, 9: 115-119.

Pintor, A.M.A., Vilar, V.J.P., Botelho, M.S., and Boaventura, R.A.R. 2016. Oil and grease removal from wastewater: sorption treatment as an alternative to state-of-the-art technologies. A critical review. Chemical Engineering Journal, 297: 229-255.

Raux, P.S., Cockenpot, H., Ramaioli, M., Quere, D., and Clanet, C. 2013. Wicking in a powder. Langmuir, 29: 3636-3644. 


\begin{abstract}
APPENDIX
PREFERENTIAL POSITIONING AND PHASE EXPOSURE OF GRANULAR PARTICLES AT HYDROPHOBIC LIQUID-WATER INTERFACE
\end{abstract}

Boglaienko, D., and Tansel, B. 2017. Journal of Cleaner Production 142: 2629-

2636. DOI: $10.1016 /$ j.jclepro.2016.11.014

\begin{abstract}
The positioning of granular limestone and quartz particles at the hydrophobic liquid-water interfaces was evaluated and compared experimentally and analyzed by the theoretical analyses by considering the electrostatic interaction and gravitational effects. Experiments were performed with light hydrophobic liquids (tetradecane, silicone oil with viscosities 10 and $100 \mathrm{cSt}$, and South Louisiana crude oil) which were submerged by granular limestone and quartz with different particle sizes (average particle radius 0.25 , $0.10,<0.004 \mathrm{~mm})$. The particles of similar size but different materials (i.e., limestone, quartz) exhibited noticeably different behavior within the hydrophobic phase and in their positioning and transport towards the hydrophobic liquid-water interface. The comparison of the dimensionless Bond number for the systems evaluated showed discrepancies between the Bond number theory and experimental observations. The preferential behavior of less than millimeter sized granular particles at the hydrophobic liquid-water interface was found to be predominantly affected by the electrostatic force only.
\end{abstract}




\section{Introduction}

Granular materials (i.e., sand, limestone) possess an ability to aggregate with a hydrophobic phase (oil), capturing significant amounts of oil that floats on a water surface. The phenomenon of selective positioning (aggregation with oil) of granular materials at the non-colloidal scale at the hydrophobic liquid-water interfaces has not been extensively studied and reported in the scientific literature. However, it is a topic of an interest as the understanding of the granular particle behavior is required for the possibility of using readily available granular materials for capturing oil and controlling mobility of floating oils as a treatment method (Abkarian et al., 2013; Boglaienko and Tansel, 2015; Boglaienko and Tansel, 2016). A number of studies have been conducted with monolayers of particles at the hydrophobic liquid-water interfaces. The reported findings and theoretical analyses were either based on or developed for colloidal systems (particulate material of 0.01-0.05 mm or a few micrometers in size) (Tavacoli et al., 2012; Horozov et al., 2003; Nikolaides et al., 2002) or nanoscale particles (Kralchevsky et al., 1994; Danov et al., 2006). It is important to report the findings of our study as there are significant differences in the particle behavior at the hydrophobic liquid-water interface when the particles are of granular size. This study focuses on the behavior and positioning of relatively larger size particles $(0.20-0.50 \mathrm{~mm}$ in diameter) after they were introduced to a

floating hydrophobic liquid phase and submerged in water (forming a particle-hydrophobic liquid aggregate).

Gravitational and electric fields act on a particle in a given conventional space and time system. Prevalent action of one or the other is related to the particle mass (size). The 
magnitude of the gravitational force can be expressed by the Newton's law of universal gravitation:

$$
F=\frac{G m M}{r^{2}}
$$

The magnitude of the electrostatic interaction between the particles can be expressed by the Coulomb's law:

$$
F=\frac{k q Q}{r^{2}}
$$

The two laws have similarities but they are of different nature. Newton's law describes the interaction between bodies with particular masses (equation 1) and Coulomb's law describes the interaction between static electrically charged particles (equation 2) over the distance $r$. Unlike the gravitational force, electrostatic (Coulomb) force can be either attractive or repulsive (depending on the charges).

The balance of the forces acting on a particle in liquid media can be generally expressed as the summation of the gravitational and electrostatic forces as follows:

$$
F^{T}=F^{E}+F^{G}
$$

For systems with very small particles (e.g., particles of colloidal scale) in liquid media, gravitational force is neglected and the particle-interface interaction is based on the interaction of electric charges only $\left(F^{T}=F^{E}\right)$. This type of particle interaction forces is referred as electrostatic image force or electrodipping force. Electrostatic image force causes attraction of a particle to the oil-water interface and electrodipping force pushes a particle into the water phase (Tavacoli et al., 2012; Danov et al., 2004). In this study, for simplicity of descriptions we will call the force that affects positioning of a particle relative to the hydrophobic liquid-water interface as electrostatic force. 
Electrostatic force acting on a particle can be expressed as (Danov et al., 2006):

$$
F^{E}=\frac{\beta_{23} Q^{2}}{4 \epsilon_{2}(R+s)^{2}} f
$$

In order to make a change from the Gauss (CGSE) system of units to the International

System of Units (SI), we express the force as follows:

$$
F^{E}=\frac{\beta_{23} Q^{2}}{16 \pi \epsilon_{0} \epsilon_{2}(R+s)^{2}} f
$$

where $R$ is the particle radius (m); $s$ is the distance from the oil-water interface (m) as depicted in Fig. $1 ; \epsilon_{0}$ is permittivity of free space $\left(8.8542 \cdot 10^{-12} \mathrm{~F} / \mathrm{m}\right) ; \epsilon_{2}$ is the dielectric constant of a hydrophobic phase; $f$ is dimensionless force coefficient; and $\beta_{23}$ is a dimensionless parameter; and $Q$ is the total surface charge of a spherical particle.
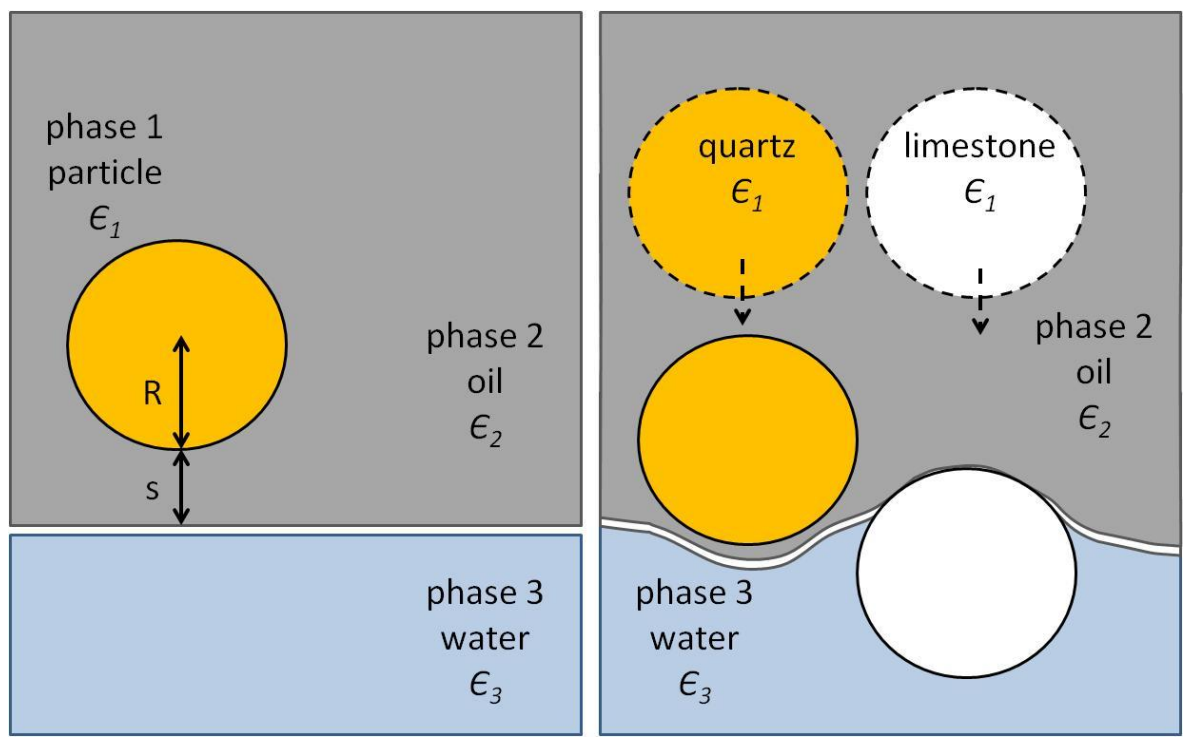

Figure 1. Schematic representation of the system with a particle and the oil-water interface (adapted from Danov et al., 2006). The particle (phase 1) of a radius $R$ is immersed in oil (phase 2) at the distance $s$ from the oil-water interface, water (phase 3). $\epsilon_{1}, \epsilon_{2}$, and $\epsilon_{3}$ are dielectric constants of particle, oil, and water phases respectively. 
In similar studies (Abkarian et al., 2013 and Tavacoli et al., 2012) that involved encapsulation of hydrophobic liquids by particles, Bond number (which compares the ratio of gravitational force acting on a particle in liquid phase to surface tension of the liquid) was the principal approach used for the numerical analyses and modeling. We also compared the relative importance of the gravitational force on the orientation of particles at the hydrophobic liquid-water interfaces (in view of the particle preferences towards one phase) using the dimensionless Bond number. The values of Bond number $(B o)$ were calculated for three hydrophobic liquids and two types of granular materials (lime stone and quartz) with fine and medium particle sizes as follows:

$$
B o=\frac{g \Delta \rho D^{2}}{\gamma}
$$

where $g$ is gravitational acceleration $\left(\mathrm{m} / \mathrm{s}^{2}\right) ; \Delta \rho$ is difference in densities of a granular material and a hydrophobic liquid $\left(\mathrm{kg} / \mathrm{m}^{3}\right) ; D$ is diameter of a particle $(\mathrm{m})$; and $\gamma$ is surface tension (in our case interfacial tension) of a hydrophobic liquid with water $(\mathrm{N} / \mathrm{m})$.

The purpose of this study was to analyze the observed differences in the behavior and positioning of natural granular particles (quartz and limestone) at the hydrophobic liquid-water interfaces and to evaluate the dominant force impact on the particles behavior. We conducted experiments using limestone and dyed quartz particles with $0.20 \mathrm{~mm}$ (fine) and $0.50 \mathrm{~mm}$ (medium) in diameter on average. The particles with different colors were used so that positioning of particles with different sizes could be visually observed in the hydrophobic liquid-water systems. The behavior of the particles at the hydrophobic liquid (tetradecane, silicone oil, and crude oil) and water interfaces were analyzed by application of the electrostatic image force theory. Relative importance of the gravitational force and 
interfacial tension on the orientation of particles at the hydrophobic liquid-water interfaces was compared using the dimensionless Bond number.

\section{Materials and Methods}

Experiments were performed with tetradecane (obtained from Fisher Scientific, purity $>99.0 \%$ ), silicone oils of viscosities 10 and $100 \mathrm{cSt}$ (obtained from Acros Organics), and South Louisiana crude oil (obtained from BP America Production Company, Houston, TX) as the hydrophobic liquids. Particles used in the experiments represent the natural granular materials, limestone and quartz sand. Colored (green and brown) quartz sand was used for the ease of observations and identification of different particle sizes during the tests when particles were applied as a mixture limestone and quartz sand or as a mixture of quartz sand with different particle sizes. Granular materials were classified by sieving using standard sieves No. 30, 40, 60 and 80 to obtain medium particle size $(<0.60 \mathrm{~mm}$ and $>$ $0.43 \mathrm{~mm}$ opening), labelled as L40 and Q40, and fine particle size $(<0.25 \mathrm{~mm}$ and $>0.18$ mm opening), labelled as L80 and Q80, for limestone (L) and quartz sand (Q). Compositions of granular materials were analyzed by Energy Dispersive Spectroscopy (Field Emission SEM JEOL 6330 with EDS “Thermo”). Experiments were performed with tap water and deionized water (room temperature $20-22{ }^{\circ} \mathrm{C}$ ). There was no difference in the aggregation phenomenon observed between tap water and deionized water, and the results reported here are given for the experiments conducted with tap water. The fact that electric force, which is caused by charges, $\sigma$, and pushes a particle to water phase, does not depend on salinity, or $\mathrm{NaCl}$ concentrations, Danov et al. (2004), supports the use of tap water. 
A 50-mL beaker was filled with $40 \mathrm{~mL}$ of tap water; $0.5 \mathrm{~mL}$ hydrophobic liquid was added on the water surface, using a 5-mL graduated pipette. The thickness of the floating hydrophobic liquid phase $(1.6 \pm 0.1 \mathrm{~mm})$ was controlled using a funnel with a cylindrical section which was partially submerged to the water. Hydrophobic liquid was added to the water surface and remained floating within the cylindrical section of the funnel, so that the thickness of the floating hydrophobic liquid could be maintained uniform before the particle addition. Total amount of granular material applied to the surface of the floating hydrophobic liquid was $1.0 \pm 0.1 \mathrm{~g}$.

Selected characteristics of the granular materials and physical properties of the liquids used during the experiments are provided in Table 1 and Table 2. The visual and SEM images of the granular materials are presented in Fig. 2.

Table 1. Characteristics of the granular materials.

\begin{tabular}{|c|c|c|c|}
\hline Granular material & $\begin{array}{c}\text { Average particle } \\
\text { radius } \\
(\mathrm{mm})\end{array}$ & $\begin{array}{c}\text { Dielectric } \\
\text { constant }^{\mathrm{a}}, \epsilon\end{array}$ & $\begin{array}{c}\text { Atomic } \\
\text { composition }^{\mathrm{b}} \\
(\%, \mathrm{w} / \mathrm{w})\end{array}$ \\
\hline $\begin{array}{l}\text { Limestone, medium } \\
\text { (L40) }\end{array}$ & 0.25 & 7 & $\begin{array}{c}\text { O } 53.10 \\
\text { Si } 19.18 \\
\text { Al } 1.56 \\
\text { Ca } 26.15 \\
\text { Total } 100\end{array}$ \\
\hline $\begin{array}{l}\text { Limestone, fine } \\
\text { (L80) }\end{array}$ & 0.10 & 7 & $\begin{array}{c}\text { O 53.10 } \\
\text { Si } 19.18 \\
\text { Al } 1.56 \\
\text { Ca 26.15 } \\
\text { Total } 100\end{array}$ \\
\hline $\begin{array}{l}\text { Quartz sand, medium } \\
\text { (brown) } \\
\text { (Q40) }\end{array}$ & 0.25 & 4 & $\begin{array}{c}\text { C } 41.72 \\
\text { O } 34.00 \\
\text { Al } 2.82 \\
\text { Na } 1.11 \\
\text { Si } 19.06 \\
\text { K } 1.30 \\
\text { Total } 100\end{array}$ \\
\hline
\end{tabular}




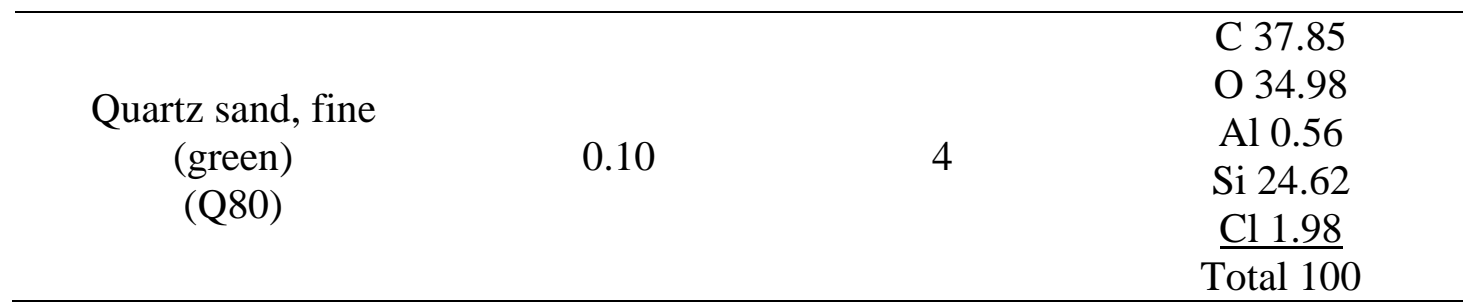

${ }^{a}$ Hubbard et al., 1997.

${ }^{\mathrm{b}}$ Determined by Energy Dispersive Spectroscopy (EDS).

Table 2. Selected physical properties of the liquids.

\begin{tabular}{ccccc}
\hline Liquid & $\begin{array}{c}\text { Viscosity } \\
(\mathrm{cSt})\end{array}$ & $\begin{array}{c}\text { Density } \\
\left(\mathrm{kg} / \mathrm{m}^{3}\right)\end{array}$ & $\begin{array}{c}\text { Interfacial tension } \\
\text { with water } \\
(\mathrm{mN} / \mathrm{m})\end{array}$ & $\begin{array}{c}\text { Surface } \\
\text { tension } \\
(\mathrm{mN} / \mathrm{m})\end{array}$ \\
\hline Tetradecane & $2.1^{\mathrm{a}}$ & $758^{\mathrm{a}}$ & $52.2^{\mathrm{b}}$ & $26.2^{\mathrm{a}}$ \\
\hline Silicone oil & 10 & $930^{\mathrm{c}}$ & $45.8^{\mathrm{d}}$ & $20.1^{\mathrm{e}}$ \\
\hline $\begin{array}{c}\text { South Louisiana } \\
\text { crude oil }\end{array}$ & $1005^{\mathrm{c}}$ & $46.2^{\mathrm{d}}$ & $20.9^{\mathrm{f}}$ \\
\hline Water & $856^{\mathrm{f}}$ & $15.5^{\mathrm{f}}$ & $26.1^{\mathrm{f}}$ \\
\hline
\end{tabular}

\footnotetext{
${ }^{a}$ Yaws, 1999.

${ }^{\mathrm{b}}$ Demond and Lindner, 1993.

c Power Chemical, Silicone oil, 2006.

${ }^{\mathrm{d}}$ Padron Aldana, 2005.

e Sigma Aldrich, Silicone oils, 2016.

${ }^{\mathrm{f}}$ US EPA, 2003.

${ }^{\mathrm{g}}$ Water-air at $25^{\circ} \mathrm{C}$.
} 

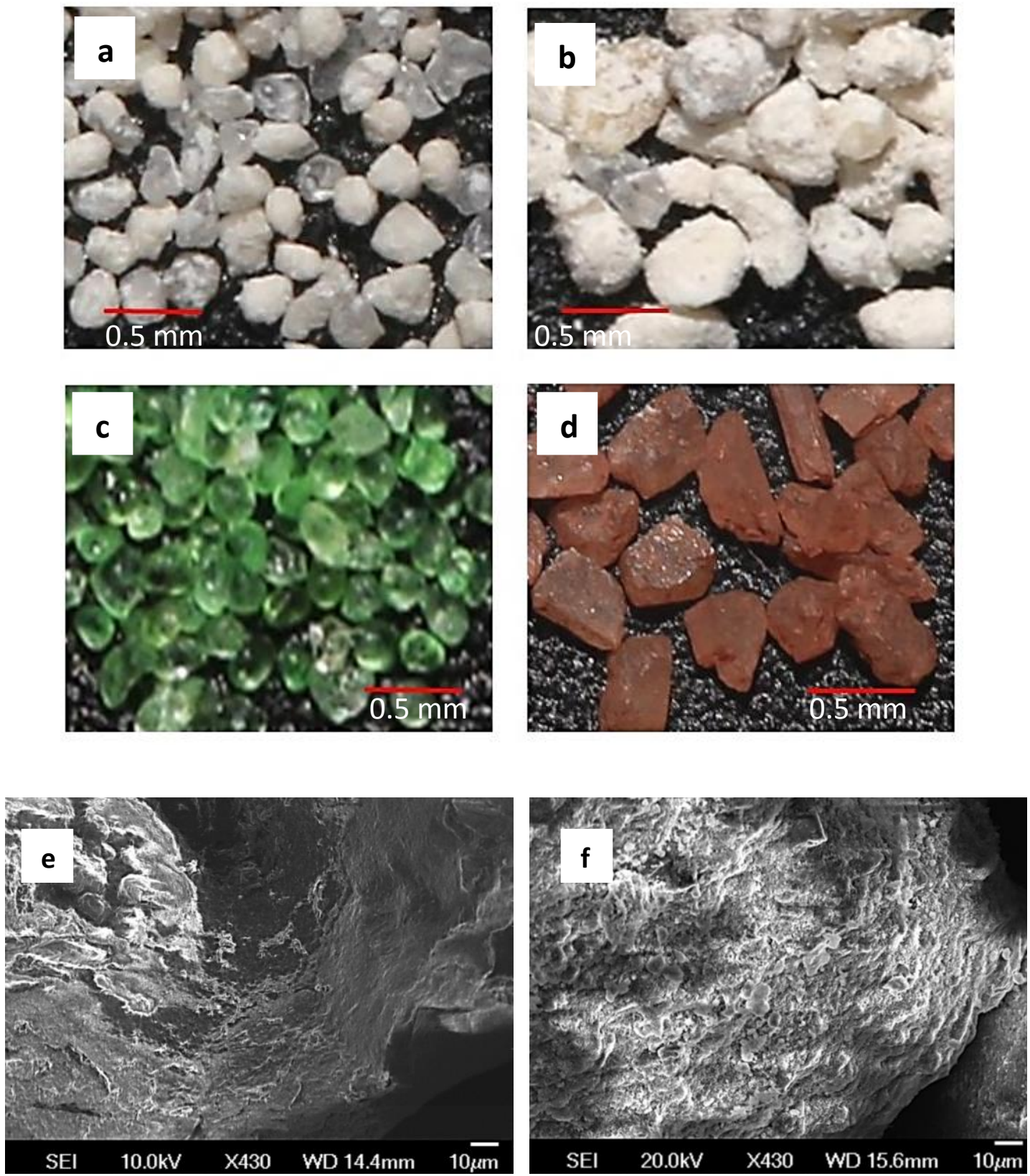

Figure 2. Granular materials: a. fine limestone, L80; b. medium limestone, L40; c. dyed fine quartz, Q80; d. dyed medium quartz, Q40; e. SEM image of fine quartz, Q80, and f. SEM image of fine limestone, L80. 


\section{Results}

Electrostatic force impact

Theoretical calculations for the electrostatic force were based on the methodology provided in Danov et al., 2006.

If electrostatic force $F^{E}>0$, the hydrophobic liquid-water boundary repels the particle (i.e., distance from the oil-water interface, $s$, increases), and if $F^{E}<0$, the phase boundary attracts the particle (i.e., distance from the oil-water interface, $s$, decreases). The sign of $F^{E}$ is defined by $\beta_{23}$, which is negative in our case.

The numerical results supported the observations for the series of experiments conducted. In our case $\epsilon_{1}>\epsilon_{2}, \beta_{12}>0$, and $f>1$ for both granular materials used (quartz and limestone) (Fig. 3a). It means that force coefficient $f$ (and electrostatic force) increases with the larger value of $\beta_{12}$, which is 0.32 for quartz and 0.55 for limestone. Electrostatic force as a function of the dimensionless distance was plotted for the quartz $\left(\epsilon_{1}=4\right)$ and limestone $\left(\epsilon_{1}=7\right)$ particles with two different particles sizes (with radii $0.10 \mathrm{~mm}$ and 0.25 mm) (Fig. 3b).

The effect of the electrostatic forces acting on a limestone particles with fine (L80) and medium (L40) sizes was significantly stronger in comparison to those experienced by the quartz particles of similar sizes (fine: Q80 and medium: Q40) as presented in Fig. 3b. This observation implied that the limestone particles were attracted to the tetradecanewater interface by the larger force than the quartz particles. The experimental observations showed that the limestone particles were not only attracted to the interface but left the hydrophobic phase (expelled) and transferred to the water phase, whereas quartz particles remained in the tetradecane phase (Table $3 \mathrm{a}, \mathrm{b}$ ). Within proximity to the interface (small 
$s / R$ ratio), the force values for fine limestone ( $280:-0.24 \mathrm{mN}$ ) are very different from the medium limestone (L40: $-1.51 \mathrm{mN}$ ). However, regardless of their size, limestone particles behaved similarly (i.e., both fine and medium sizes were expelled from the hydrophobic phase), suggesting that there is a critical range of values of electrostatic force, below which particles prefer to transfer to the water phase. Quartz particles of both particle sizes remained within tetradecane phase (Table 3c) and their preferential positioning did not depend on the particle size as well.

The behavior of the limestone particles in crude oil differed significantly (Table $3 \mathrm{~d}$ and 3e) as they did not leave the hydrophobic liquid-water interface, but remained at the distance close to $s=0$. The dyed quartz particles of both sizes were applied on the crude oil slick and remained submerged into the crude oil (forming and aggregate) (Table $3 f$ ).

The fact that limestone particles were held at the interface with crude oil might be due to the higher viscosity of the crude oil in comparison to tetradecane. Dynamic viscosity of tetradecane is almost five times lower than the viscosity of South Louisiana Crude oil (Table 2). We conducted additional experiments using silicone oil with different viscosities to test this hypothesis. As the images in Table 3g-j show, the behavior of limestone and quartz particles in silicone oil with $100 \mathrm{cSt}$ viscosity was very similar to that one observed with tetradecane for both particle sizes. Based on the experimental observations, viscosity of the hydrophobic liquid did not affect the positioning of the particles.

As shown in Fig. 2, surface of limestone is soft (Fig. 2a-d) and not as smooth as quartz sand used (Fig. 2e, f) with some small particles (nano scale range of sizes) detaching from limestone due to contact and friction between the particles. When very small limestone particles (average particle radius $<0.004 \mathrm{~mm}$ ) were applied to the hydrophobic 
phase (tetradecane), we observed that they were able to create globules of tetradecane floating just below the water surface (Table $3 \mathrm{k}$ ). The hydrophobic phase of tetradecane did not submerge entirely because the amount of limestone present (forming an encapsulation) was not sufficient to overcome the gravitational forces. Unlike the particles with larger sizes, very fine (micro to nano scale) limestone particles exhibited very different behavior. Noticeable differences in the behavior of micro scale and micro/nano scale limestone particles depicted by images (l) and (m) in Table 3, where a mixture of limestone with medium and very fine particle sizes was applied on the floating silicone oil in water. The submerged globules of hydrophobic phase were selectively encapsulated by only very fine limestone particles, with medium size particle being expelled from the hydrophobic phase. 

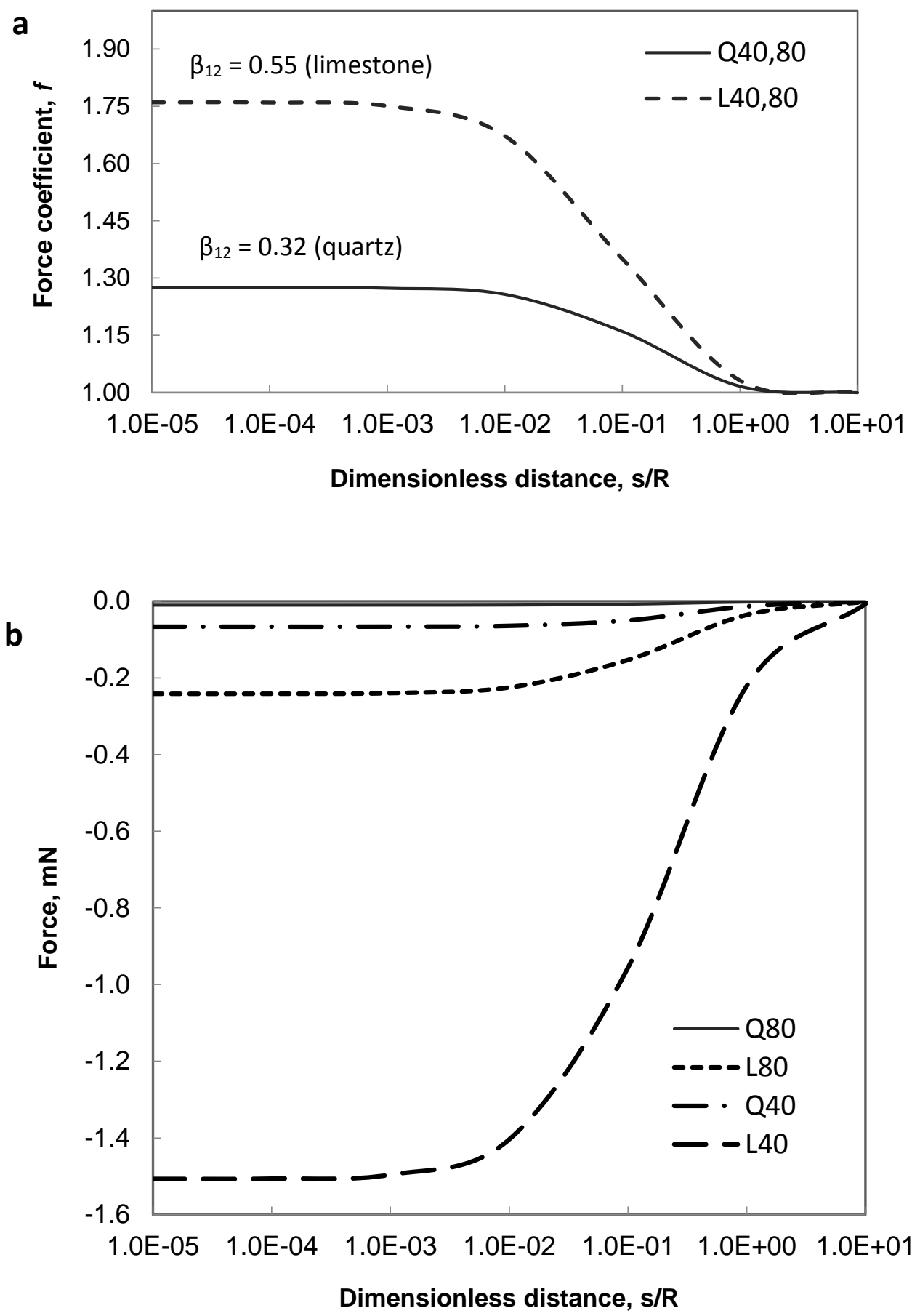

Figure 3. a. The force coefficient for the limestone and quartz sand particles in tetradecane. The dimensionless distance $s / R$ represents the relation of a particle to the tetradecane-water interface ( $s$ : distance to interface, $R$ : Particle radius); $\beta_{23}=-0.95$ (attraction to the tetradecane-water interface). b. Interaction energies of particles with 
different radii as a function of the dimensionless distance. Line Q80: fine quartz particles, $R_{1}=0.10 \mathrm{~mm}$; line Q40: medium quartz particles, $R_{2}=0.25 \mathrm{~mm}$; line L80: fine limestone particles, $R_{l}=0.10 \mathrm{~mm}$; and line L40: medium limestone particles, $R_{2}=0.25 \mathrm{~mm}$.

Table 3. Preferential positioning of granular particles at the hydrophobic liquid-water interface.

\begin{tabular}{|c|c|c|c|}
\hline $\begin{array}{l}\text { Particle-interface preferential } \\
\text { positioning observations* }\end{array}$ & Particles and liquids & $\begin{array}{l}\text { Average } \\
\text { Particle } \\
\text { radius } \\
(\mathrm{mm})\end{array}$ & $\begin{array}{l}\text { Dominance of } \\
\text { electrostatic force } F^{E} \text { of } \\
\text { the granular materials }\end{array}$ \\
\hline & $\begin{array}{c}\text { Dyed fine quartz Q80 } \\
\text { Fine limestone L80 } \\
\text { Tetradecane }\end{array}$ & $\begin{array}{l}0.10 \\
0.10\end{array}$ & $F^{E} L 80 \gg F^{E} Q 80$ \\
\hline b & $\begin{array}{c}\text { Dyed medium quartz Q40 } \\
\text { Medium limestone L40 } \\
\text { Tetradecane }\end{array}$ & $\begin{array}{l}0.25 \\
0.25\end{array}$ & $F^{E} L 40>>F^{E} Q 40$ \\
\hline (1) & $\begin{array}{c}\text { Dyed fine quartz Q80 } \\
\text { Dyed medium quartz Q40 } \\
\text { Tetradecane }\end{array}$ & $\begin{array}{l}0.10 \\
0.25\end{array}$ & $F^{E} Q 80 \approx F^{E} Q 40$ \\
\hline $10 . . .$. & $\begin{array}{c}\text { Dyed fine quartz Q80 } \\
\text { Fine limestone L80 } \\
\text { Crude oil }\end{array}$ & $\begin{array}{l}0.10 \\
0.10\end{array}$ & $F^{E} L 80>F^{E} Q 80$ \\
\hline
\end{tabular}




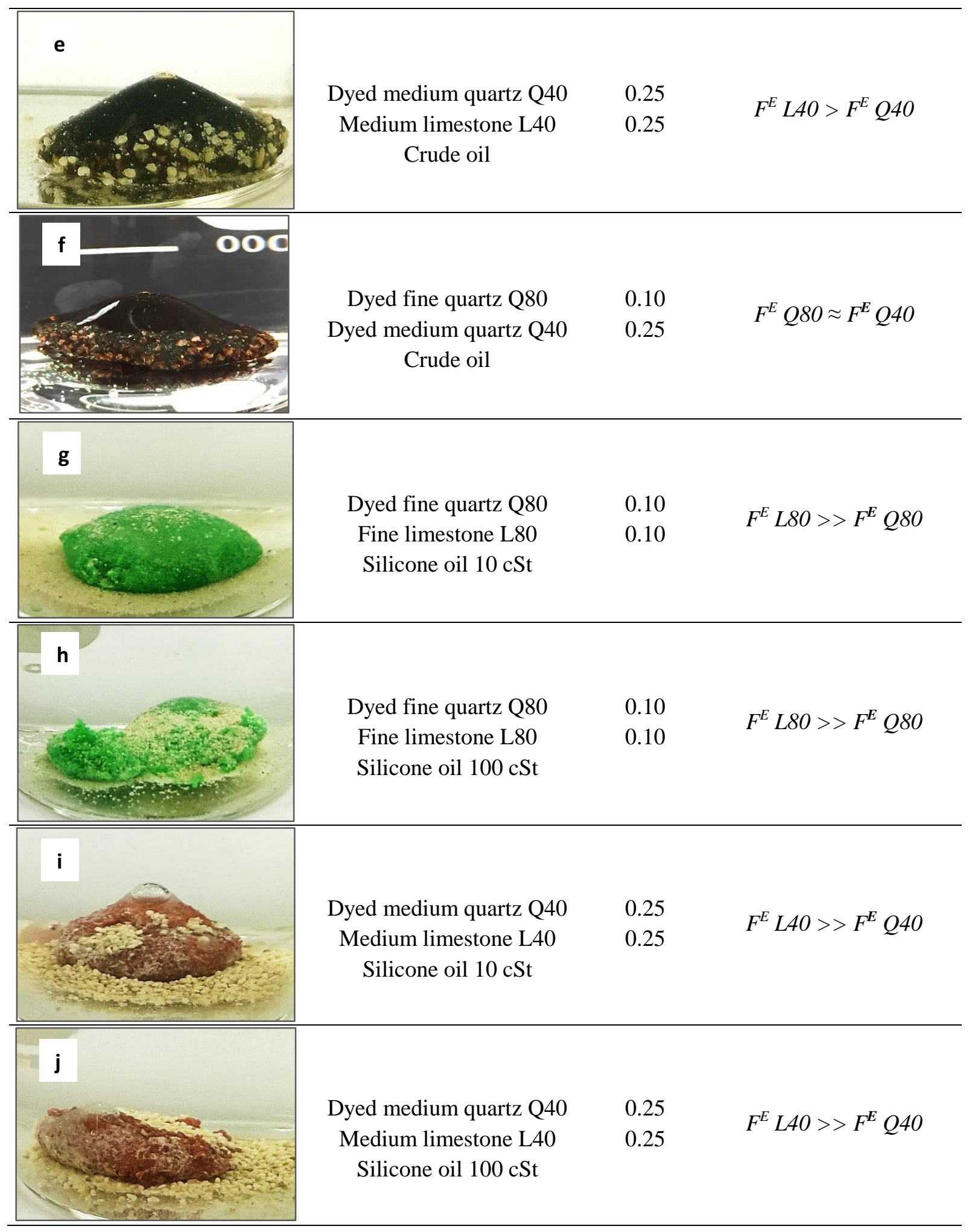




$\begin{gathered}\text { Very fine limestone } \mathrm{L}<200 \\ \text { Tetradecane }\end{gathered}$
$\begin{gathered}\text { Very fine limestone } \mathrm{L}<200 \\ \text { Medium limestone } \mathrm{L} 40 \\ \text { Silicone oil } 10 \mathrm{cSt}\end{gathered}$

* Experimental results for the numerical analysis: quartz and limestone particles submerged into tetradecane (images a, b, c) and crude oil (images d, e, f) in water. Images (a) and (d), and (b) and (e) are mixtures of quartz and limestone particles of the same size applied simultaneously on the floating on water hydrophobic phase. Images (c) and (f) present a mixture of quartz particles of the two different sizes applied simultaneously on the floating on water hydrophobic phase. Images (g) and (i) illustrate quartz and limestone particles submerged into silicone oil of $10 \mathrm{cSt}$, and images (h) and (j) show similar for silicone oil of $100 \mathrm{cSt}$. Globules of tetradecane in water encapsulated with very fine limestone are presented in image $(\mathrm{k})$. Positioning of limestone particles of very fine and larger sizes applied at the floating silicone oil in water is illustrated by images (l) and (m). 


\section{Gravitation force impact}

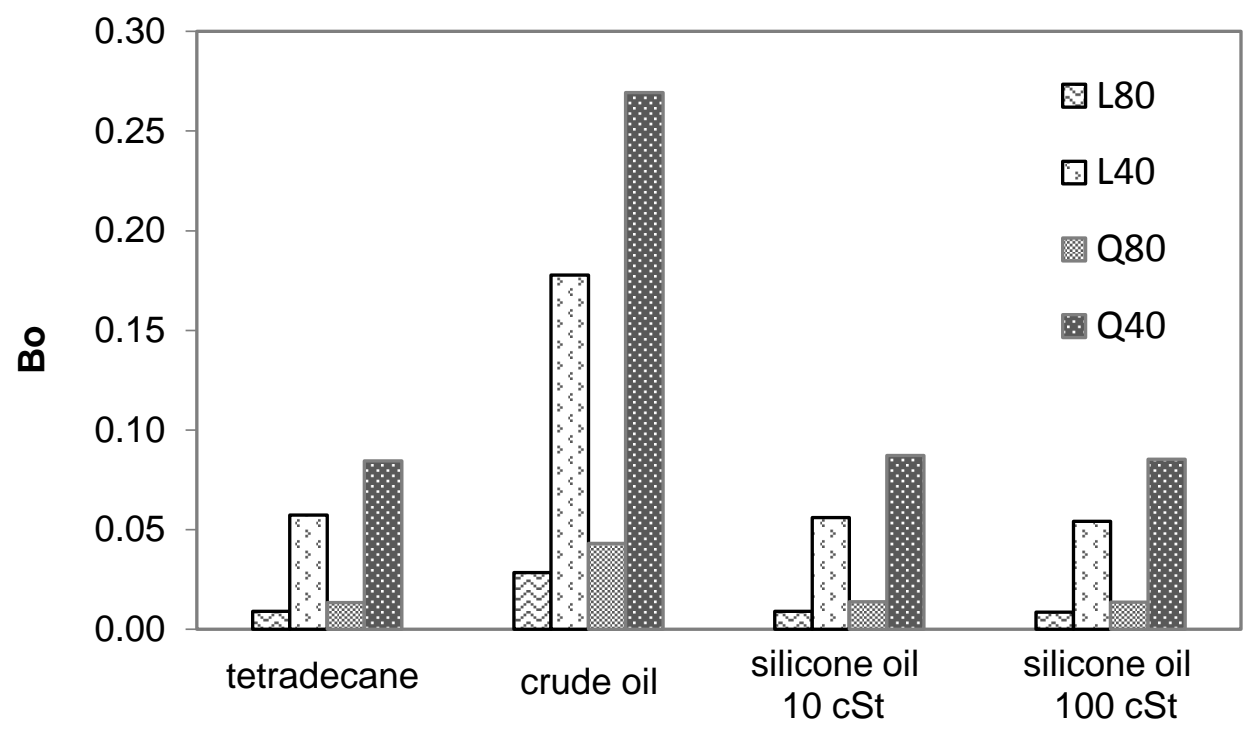

Figure 4. Comparison of the Bond numbers for the granular materials with different sizes submerged into the hydrophobic phase. (Q80: fine quartz particles, $R_{1}=0.10 \mathrm{~mm}$; Q40: medium quartz particles, $R_{2}=0.25 \mathrm{~mm}$; L80: fine limestone particles, $R_{1}=0.10 \mathrm{~mm}$; and L40: medium limestone, $R_{2}=0.25 \mathrm{~mm}$ ).

As presented in Fig. 4, Bond numbers for the limestone particles of both sizes in the tetradecane phase are lower than those for the quartz particles due to lower density of limestone than quartz (1980 and $2560 \mathrm{~kg} / \mathrm{m}^{3}$ for limestone and quartz, respectively). The smaller values of Bond number indicate lower gravitational forces acting on a particle in comparison to forces due to interfacial tension. However, the experimental observations (Table 3) showed the opposite behavior, such that the limestone particles crossed the interface (i.e., moved from the hydrophobic phase to the water phase) despite the gravitational force acting on them is smaller than the gravitational force acting on the quartz 
particles. Additionally, Bond numbers for crude oil are higher (due to lower interfacial tension), however, limestone particles remained at the interface. We observed that, when the effect of gravitational force is negligible, Bond number does not adequately describe the behavior of a particle at the hydrophobic liquid-water interface and may not be appropriate for analysis of such systems (i.e., for particles with micro and nano scale diameters). The study by Danov et al. (2004) describes that even for particles as large as $0.40-0.60 \mathrm{~mm}$ in diameter at the oil-water interface, the interfacial deformation is dominated by the forces of electric nature, not gravitational, or $F^{E}>F^{G}$ (e.g., glass particles at the tetradecane-water interface). However, for particles that are millimeter and larger, gravitational force becomes dominant and Bo number has more relevancy.

\section{Discussion}

Based on the comparison of the experimental observations and theoretical analyses of the electrostatic force and the gravitational effects on the granular particles, the theoretical results did not support the assumption that particle positioning at the liquid interface can be theoretically linked to gravitational force (heavier particles of quartz stayed within the hydrophobic phase while limestone particles did not). Electrostatic force would cause predominant effect on the positioning of a particle, $F^{E}>>F^{G}$ then $F^{T}=F^{E}$.

The possible explanation for the observed phenomenon of variations in preferential positioning of the granular materials may relate to the differences in surface charge and reactivity between the quartz and limestone particles. Carbonate surface group interacts with up to three water molecules (Wolthers et al., 2012), whereas in natural quartz particles (sand) the $\mathrm{SiO}_{2}: \mathrm{H}_{2} \mathrm{O}$ ratio is less than 1:2 (Júnior and Baldo, 2014). The dyed sand had 
carbon content around $40 \%$ by weight (Table 1) which decreased the reactivity of its surface in comparison to that of pure quartz. The difference in surface charges (and electrostatic forces) of dyed sand and limestone becomes very significant, hence, affects the positioning behavior of the particles. Limestone, which has active surface properties (in comparison to quartz which is inert) possesses higher surface charge, or higher zeta potential. Therefore, limestone particles were able to cross the tetradecane-water interface, leaving the non-reactive tetradecane phase. Zeta potential of aqueous limestone decreased with the addition of crude oil (Bassioni and Taqvi, 2015) and that is why limestone particle were held at the crude oil-water interface. Polar fractions of crude oil (asphaltenes and resins) affect zeta potential and surface charge of a particle, as asphaltenes possess positive charges and resins are negatively charged (Gonzales et al., 2003). Thus, the surface of limestone particles, being submerged into crude oil, became less reactive with water molecules, having polar molecules of crude oil adsorbed to it. As a result, limestone particles remained submerged within the crude oil phase.

In case of larger limestone particles, due to their relatively high surface reactivity with water, they were driven through hydrophobic liquid-water interface, and able to cross the interface and transfer to the water phase. However, very fine limestone particles were of the same nature but remained attached at the interface (Table 3k-m) as their total surface charge strongly decreases with size $\left(Q \sim R^{2}\right)$. From this perspective, electrostatic force's relative effect, as the major force that controls the positioning of the particles in different hydrophobic liquids, is presented in Table 3.

It is important to note that slight differences in material composition (dyed quartz sand versus pure quartz) affect surface characteristics, resulting in differences in 
preferential positioning of particles. The experimental observations showed that the effect of the charge difference is more significant than the effect of the size difference in determining the position of a particle in a hydrophobic liquid, hydrophobic liquid-water interface, and water phase.

\section{Conclusions}

The behavior of the quartz and limestone particles in the submerged hydrophobic phase was evaluated and compared with theoretical analyses which included considerations of the electrostatic interactions and gravitational force. Particles showed noticeably different behavior in their phase preference depending on their size and material type. Quartz particles preferred to remain within the hydrophobic phase (average particle radii 0.25 and $0.10 \mathrm{~mm}$ ), whereas the behavior of limestone particles of the same size was the opposite, except for the very fine particles (average radius $<0.004 \mathrm{~mm}$ ) and when the hydrophobic phase was crude oil. As the effect of gravitational force was negligible, Bond number, which was often used for such systems, did not adequately describe the behavior of a particle at the hydrophobic liquid-water interface. The particles' positioning effect was related to electrostatic force (surface charge) and reactivity differences of the granular materials.

The phenomenon we study is of high interest as it presents a novel oil spills treatment method, which is simple, inexpensive and environmentally friendly. 


\section{Acknowledgments}

This research was partially supported by Gulf of Mexico Research Initiative (GoMRI) through funding to Consortium for the Molecular Engineering of Dispersant Systems (CMEDS) under RFP-I: Consortia Grants and by Dissertation Year Fellowship (Florida International University). We thank Dr. Piero Gardinali for the helpful discussion.

References

Abkarian, M., Protiere, S., Aristoff, J.M., and Stone, H.A. 2013. Gravity-induced encapsulation of liquids by destabilization of granular rafts. Nature Communications, 4 (1895).

Bassioni, G., and Taqvi, S.T. 2015. Wettability studies using zeta potential measurements. Journal of Chemistry, 2015.

Boglaienko, D., and Tansel, B. 2015. Instantaneous stabilization of floating oils by surface application of natural granular materials (beach sand and limestone). Marine Pollution Bulletin, 91: 107-112.

Boglaienko, D., and Tansel, B. 2016. Gravity induced densification of floating crude oil by granular materials: effect of particle size and surface morphology. Science of The Total Environment, 556: 146-153.

Danov, K.D., Kralchevsky, P.A., Ananthapadmanabhan, K.P., and Lips, A. 2006. Particle - interface interaction across a nonpolar medium in relation to the production of particle-stabilized emulsions. Langmuir, 22: 106-115.

Danov, K.D., Kralchevsky, P.A., and Boneva, M.P. 2004. Electrodipping force acting on solid particles at a fluid interface. Langmuir, 20: 6139-6151.

Demond, A.H., and Lindner, A.S. 1993. Estimation of interfacial tension between organic liquids and water. Environmental Science \& Technology, 27: 2318-2331.

Gonzales, G., Neves, G.B.M., Saraiva, S.M., Lucas, E.F., and dos Anjos de Sousa, M. 2003. Electrokinetic characterization of asphaltenes and the asphaltenes-resins interaction. Energy \& Fuels, 17 (4): 879-886. 
Horozov, T.S., Aveyard, R., Clint, J.H., and Binks, B.P. 2003. Order-disorder transition in monolayers of modified monodisperse silica particles at the octane-water interface. Langmuir, 19: 2822-2829.

Hubbard, S.S., Peterson, J.E., Majer, E.L., Zawislanski, P.T., Williams, K.H., Roberts, J., and Wobber, F. 1997. Estimation of permeable pathways and water content using tomographic radar data. The Leading Edge, 16: 1623-1628.

Júnior, J.A.A., and Baldo, J.B. 2014. The behavior of zeta potential of silica suspensions. New Journal of Glass and Ceramics, 4: 29-37.

Kralchevsky, P.A., and Nagayama, K. 1994. Capillary forces between colloidal particles. Langmuir, 10: 23-36.

Nikolaides, M.G., Bausch, A.R., Hsu, M.F., Dinsmore, A.D., Brenner, M.P., Gay, C., and Weitz, D.A. 2002. Electric-field-induced capillary attraction between like-charged particles at liquid interfaces. Nature, 420: 299-301.

Padron Aldana, G.A. 2005. Effect of surfactants on drop size distribution in a batch, rotorstator mixer. Ph.D. Dissertation, University of Maryland, 2005. http://drum.lib.umd.edu/bitstream/handle/1903/2160/umi-umd2140.pdf;jsessionid=08DC914F51D799E2BC5E92774A022D7F?sequence $=1$ (accessed April 4, 2016).

Power Chemical, Silicone oil, 2006. http://www.powerchemical.net/library/Silicone_Oil.pdf (accessed April 4, 2016).

Sigma Aldrich, Silicone oils. http://www.sigmaaldrich.com/materials-science/materialscience-products.html?TablePage $=20204397$ (accessed April 4, 2016).

Tavacoli J.W., Katgert, G., Kim, E.G., Cates, M.E., and Clegg, P.S. 2012. Size limit for particle-stabilized emulsion droplets under gravity. Physical Review Letters, 108: 268306(5).

USEPA, EPA/600/R-03/072. 2003. Characteristics of spilled oils, fuels, and petroleum products: 1. Composition and properties of selected oils.

Wolthers, M., Tommaso, D.D., Du, Z., and Leeuw, N.H. 2012. Calcite surface structure and reactivity: molecular dynamics simulations and macroscopic surface modelling of the calcite-water interface. Physical Chemistry Chemical Physics, 14: 1514515157.

Yaws, C.L. 1999. Chemical properties handbook: physical, thermodynamic, environmental, transport, safety, and health related properties for organic and inorganic chemicals. McGrown-Hill Handbooks. 
VITA

\section{DARIA BOGLAIENKO}

2004 B.S. Ecology (diploma with honors)

National Technical University 'Kharkiv Polytechnical Institute'

Kharkiv, Ukraine

Thesis: Influence of the Emissions of Machine and Assembly Shop of the State Enterprise Kharkiv Engine Plant 'Serp i Molot' on the Air Basin of Moskovsky Region and Justification of the Decisions on Ecosystem Stability

2006 M.S. Ecology and Environmental Protection (diploma with honors) National Technical University 'Kharkiv Polytechnical Institute'

Kharkiv, Ukraine

Thesis: Development and Justification of the Flowsheet 'Anaerobic Digester-Diesel Generator' Supporting Mesophilic Mode in Anaerobic

Digester due to the Heat Released by Diesel Generator

2013

M.S. Environmental Studies

Florida International University

Miami, FL, USA

Thesis: Buckwheat as a Cover Crop in Florida: Mycorrhizal Status, Soil

Analysis, and Economic Assessment

2013-2017 Doctoral Candidate

Florida International University

Miami, FL, USA

\section{PUBLICATIONS}

Boglaienko, D., and Tansel, B. 2017. Wicking of light hydrophobic liquid phase from water by pulverized rubber: theoretical and experimental analyses. Journal of Hazardous Materials, 325: 189-197. DOI: 10.1016/j.jhazmat.2016.11.076

Boglaienko, D., and Tansel, B. 2017. Preferential positioning and phase exposure of granular particles at hydrophobic liquid-water interface. Journal of Cleaner Production, 142: 2629-2636. DOI: 10.1016/j.jclepro.2016.11.014

Tansel, B., and Boglaienko, D. 2017. Self assembly, mobilization, and flotation of crude oil contaminated sand particles as granular shells on gas bubbles in water. Science of Total Environment, 574: 437-442. DOI: 10.1016/j.scitotenv.2016.09.109 
Boglaienko, D., and Tansel, B. 2017. Submergence patterns of floating crude oil by granular particles. Chemical Engineering Journal, 314: 548-553. DOI: 10.1016/j.cej.2016.12.012

Boglaienko, D., and Tansel, B. 2016. Partitioning of fresh crude oil between floating, dispersed and sediment phases: Effect of exposure order to dispersant and granular materials. Journal of Environmental Management, 175: 40-45. DOI: 10.1016/j.jenvman.2016.03.017

Boglaienko, D., and Tansel, B. 2016. Gravity induced densification of floating crude oil by granular materials: effect of particle size and surface morphology. Science of The Total Environment, 556: 146-153. DOI: 10.1016/j.scitotenv.2016.01.214

Boglaienko, D., and Tansel, B. 2016. Encapsulation of light hydrophobic liquids with fine quartz sand: property based characterization and stability in aqueous media with different salinities. Chemical Engineering Science, 145: 90-96. DOI: 10.1016/j.ces.2016.02.010

Boglaienko, D., Tansel, B., and Sukop, M.C. 2016. Granular encapsulation of light hydrophobic liquids (LHL) in LHL-salt water systems: particle induced densification with quartz sand. Chemosphere, 144: 1358-1364. DOI: 10.1016/j.chemosphere.2015.10.019

Boglaienko, D., and Tansel, B. 2015. Instantaneous stabilization of floating crude oils by surface application of natural granular materials (beach sand and limestone). Marine Pollution Bulletin, 91 (1): 107-112. DOI: 1016/j.marpolbul.2014.12.020

Boglaienko, D., Soti, P., Shetty, K.G., and Jayachandran, K. 2014. Buckwheat as a cover crop in Florida: mycorrhizal status and soil analysis. Agroecology and Sustainable Food Systems, 38 (9): 1033-1046. DOI: 10.1080/21683565.2014.906016 